UC-66b

Issued: September 1981

\title{
Investigations of Low-Temperature Geothermal Potential in New York State
}

\author{
Dennis S. Hodge* \\ Robert De Rito* * \\ Kenneth Hifiker** \\ Paul Morgan ${ }^{\dagger}$ \\ Chandler A. Swanbergtt
}

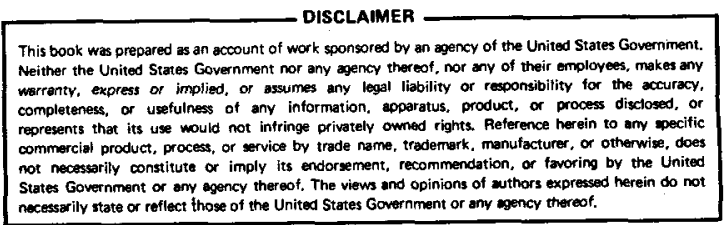

"Short-term Visiting Staff Member. State University of New York at Buffalo, Department of Geological Sciences, 4240 Ridge Lea Road, Amherst, NY 14226.

-" State University of New York at Buffalo, Department of Geological Sciences, 4240 Ridge Lea Road, Amherst, NY 14226.

tVisiting Staff Member. NASA Lunar and Planetary Institute, Houston, TX 77058

${ }^{t+}$ Visiting Staff Member. New Mexico State University, Departments of Earth Sciences and Physics, Box 3D, Las Cruces, NM 88003. 
काष

Fon
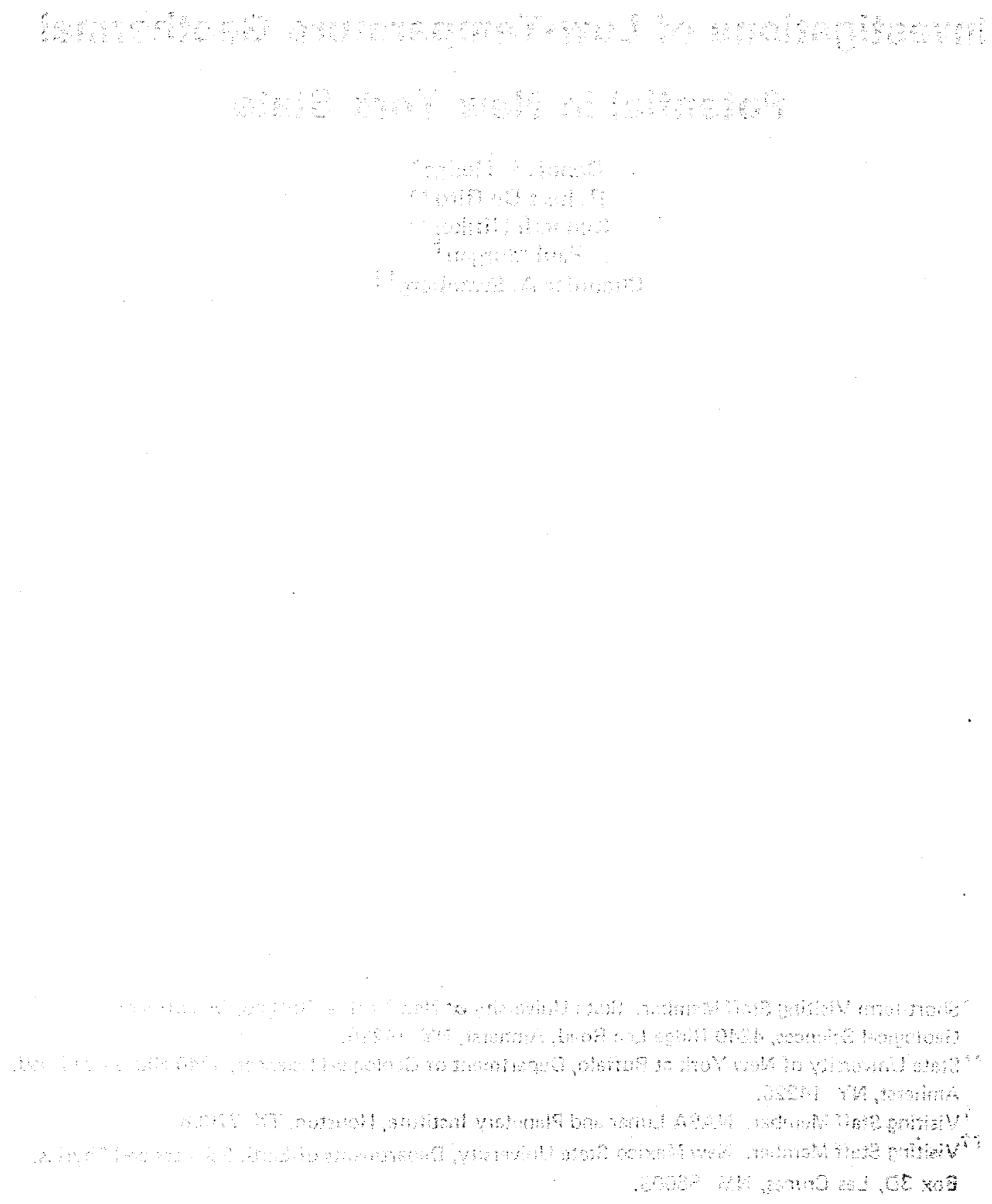

(n) 
INVESTIGATIONS OF LOW-TEMPERATURE

GEOTHERMAL POTENTIAL IN NEW YORK STATE

by

Dennis S. Hodge, Robert De Rito, Kenneth Hifiker, Paul Morgan, and

Chandler A. Swanberg

\begin{abstract}
The Association of American Petroleum Geologists (AAPG) temperature gradient map and published heat flow data indicate a possible potential for a geothermal resource in western and central New York State. A new analys is of bottomhole temperature data for New York State confirms the existence of three positive gradient anomalies: the East Aurora, Cayuga, and Elmira anomalies, with gradients as high as $32^{\circ} \mathrm{C} / \mathrm{km}$, $36^{\circ} \mathrm{C} / \mathrm{km}$, and $36^{\circ} \mathrm{C} / \mathrm{km}$, respectively. Ground waters from two of these anomalies are enriched in silica relative to surrounding areas. Heat flows based on silica geothermometry are $50-70 \mathrm{mWm}^{-2}$ for the anomalies and $41.4 \mathrm{mWm}^{-2}$ for bordering regional flux. A correlation between Bouguer gravity anomalies and the temperature gradient map suggests that the geothermal anomalies may occur above radioactive granites in the basement.
\end{abstract}

\title{
I. INTRODUCTION
}

Inspection of the Association of American Petroleum Geologists (AAPG) temperature gradient map for the US [AAPG-US Geological Survey (USGS), 1976] reveals that two of the most prominent anomalies in the eastern US are near Cayuga Lake and East Aurora, New York. Temperature gradients greater than $36^{\circ} \mathrm{C} / \mathrm{km}$ were estimated from corrected bottom-hole temperatures from oil and gas boreholes. Because these two areas are located near large population 
centers, considerable potential exists for use of geothermal energy, and an evaluation of subsurface temperatures, heat flow, and the causes of the anomalies has been undertaken.

The study focused on (1) preliminary geochemical sampling of the two possible resource areas, (2) analysis of gravity data to determine the subsurface distributions of mass, and (3) acquisition of a more complete set of bottom-hole temperature data for New York State.

The only published heat flow data for central and western New York is the work of Diment et al. (1972). As shown in Fig. 1, heat flow values for three areas near Buffalo are $50 \mathrm{~mW} / \mathrm{m}^{2}$, and values for three localities southwest of Syracuse are in the range of 60 to $70 \mathrm{mWm}^{-2}$. These values are significantly

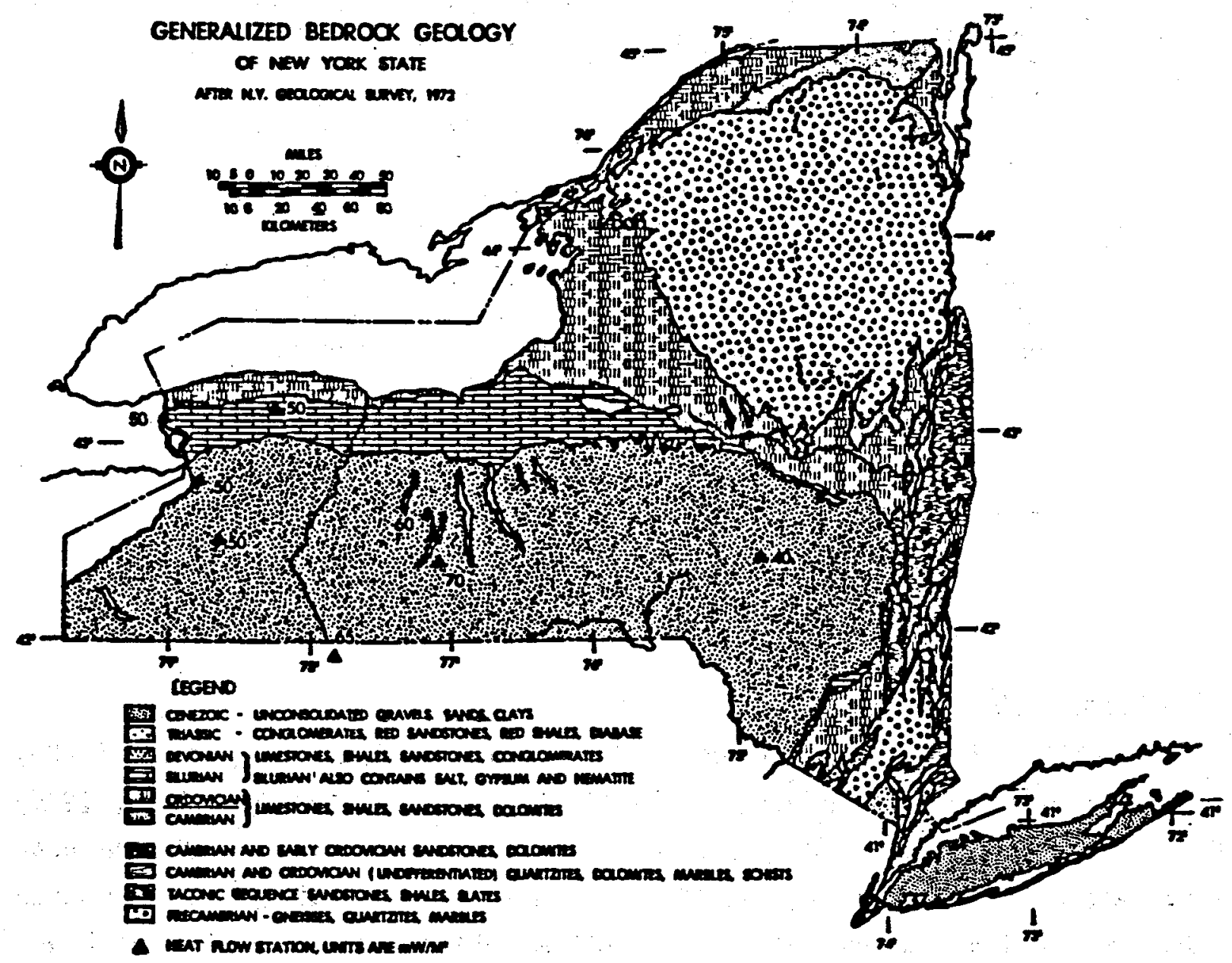

Fig. 1.

Geologic map of New York State showing that Cambrian through Devonian sediments cover the area studied in this report. Heat flow data are from Diment, Urban, and Revetta (1972). 
above the norm for the eastern. US, although Diment and others question the reliability of some of the data. The values indicate, however, that anomalously high flux may exist in restricted areas in the region.

\section{REGIONAL GEOLOGY}

The central and western portions of New York State are characterized by relatively simple geologic structure. Cambrian through Devonian shales and limestones dip gently to the south. The thickness of this sedimentary sequence ranges from $1000 \mathrm{~m}$ at the shore of Lake Ontario (Fig. 1) to over $3200 \mathrm{~m}$ in some areas to the south. Precambrian crystalline basement rocks underlie the Paleozoic sediments. Although the Paleozoic section contains some evaporites, most of it is shales and sandstones. The veneer of glacial debris that covers most of the area may be as thick as $200 \mathrm{~m}$ in some valleys.

\section{TEMPERATURE GRADIENTS}

The temperature gradient map prepared by the AAPG (1976) for the geothermal survey of North America made use of bottom-hole temperatures from approximately 125 wells in New York State. Bottom-hole temperatures have been compiled for 837 additional wells (Appendix A), and the gradients from the central and western portions of New York State have been reevaluated. Surface temperatures for the gradient calculations were estimated from mean annual temperatures compiled by the National Oceanic Atmospheric Administration (NOAA) for 73 recording stations in the State (Fig. 2). Sea level temperatures were calculated using a lapse rate of $9.8^{\circ} \mathrm{C} / \mathrm{km}$ (Jaeger 1964), and a second-order trend surface was fitted to the results (Fig. 3). The trend surface corrected to borehole collar elevation was then used to calculate the surface temperature at each well location.

The lateral variation in geothermal gradients calculated from bottom-hole temperatures is shown on contour maps. A computer code developed by the Kansas Geological Survey (Sampson, 1975) was used for contouring. Various data sets were selected and grid systems were established using a two-phase procedure.

In the first phase of gridding, a search procedure finds the four neighbors that are closest to the data point being considered. A weighted trend surface is then fitted to these four points and the central data point. The neighboring points are assigned statistical weighting factors that are 


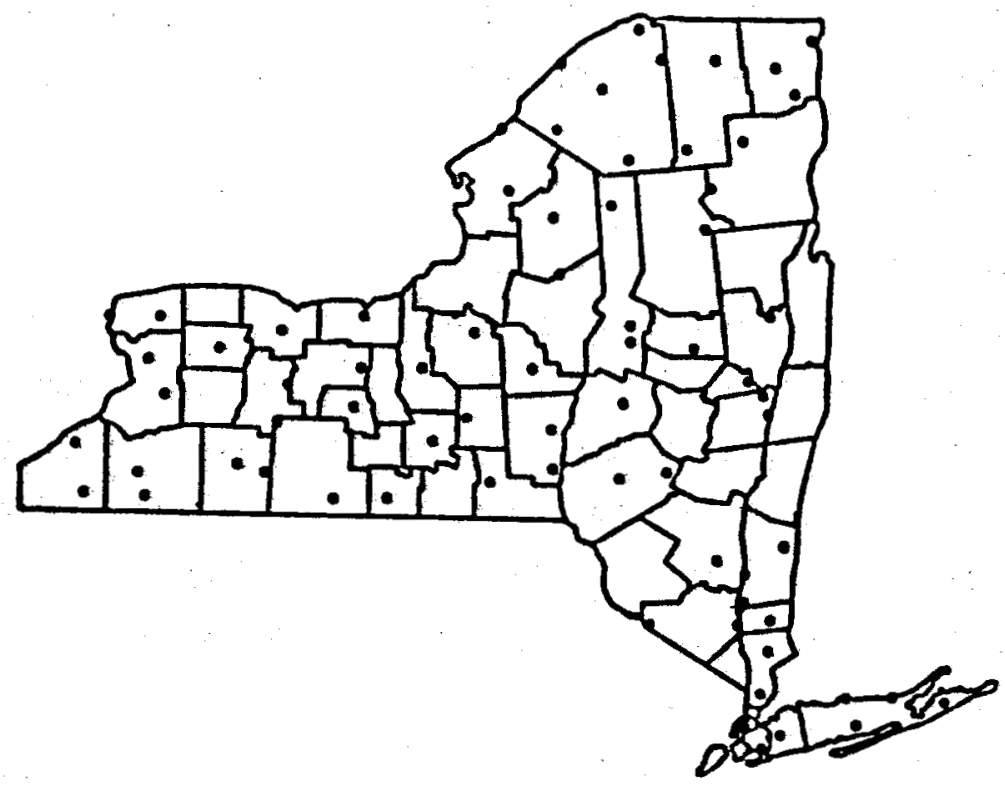

Fig. 2.

Locations of surface temperature recording stations in New York State.

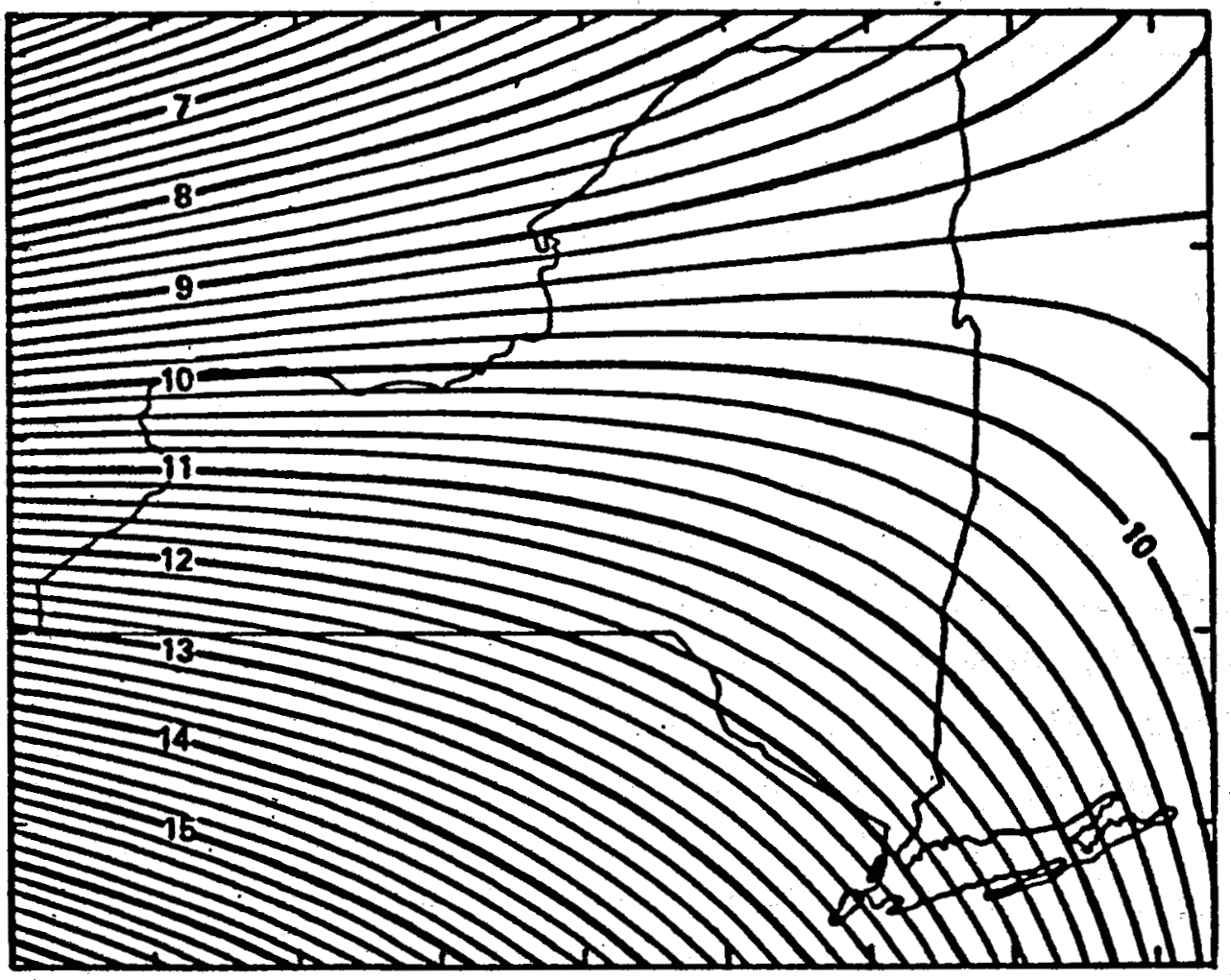

Fig. 3.

Second-order trend surface of surface temperature reduced to sea level. Temperatures in ${ }^{\circ} \mathrm{C}$. 
inversely proportional to the distance from the one being evaluated. The constant of the fitted regression is adjusted so that the plane passes through the data point under consideration. This process is repeated for all points and the coefficients of the trend surface are saved.

The second phase of gridding finds the four data points that are nearest to a node in a predetermined grid matrix. The coordinates of the node are substituted into the trend surface equation calculated for the four neighboring points. The effect is that of projecting the slopes to the node. An inverse-distance weighting procedure is again applied to the slope projections at the node, and the value there is estimated by a weighted average. If an actual data point is on or very near a grid intersection, the original value for it is used for the value at the node.

The grid matrix was then smoothed to reduce undesired noise that may be present in the system. Smoothing was done using weighted arithmetic averaging of adjacent gradient values in the grid matrix. "The weighted smoothing procedure multiplied each center point by a factor of two and averaged the result with the grid nodes in the adjacent two rows and columns of the matrix. Each adjacent grid value was assigned a weighting factor equal to the square of the inverse of its distance from the grid node being smoothed. Therefore, the more distant locations in the matrix contributed less to the smoothed point. The smoothing operation was performed for each node in the matrix. At this stage of the procedure, randomly scattered data have been replaced by a regularly spaced grid matrix. Simple linear interpolation was used to determine the point at which a contour line intersects the side of a grid cell. A series of such intersections defines a contour line path, and a set of plotting instructions was then developed and subsequently run on a calcomp model $925 / 936$ plotting system.

The above procedure was applied to 789 wells deeper than $500 \mathrm{~m}$ in western and central New york (Fig. 4) and the resulting information was contoured (Fig. 5). The geothermal gradients were calculated as the quotients of the bottom-hole temperatures minus the estimated surface temperature divided by the well depth. The bottom-hole temperatures were not corrected for drilling disturbances. The calculated geothermal gradients in the data set ranged from $10.64^{\circ} \mathrm{C} / \mathrm{km}$ to $41.85^{\circ} \mathrm{C} / \mathrm{km}$. The gradients in the computer generated grid matrix are in the range of $13.32^{\circ} \mathrm{C} / \mathrm{km}$ to $35.4^{\circ} \mathrm{C} / \mathrm{km}$, and the arithmetic smoothing process performed on the grid altered the range to $15.67^{\circ} \mathrm{C} / \mathrm{km}$ to $30.94^{\circ} \mathrm{C} / \mathrm{km}$. 


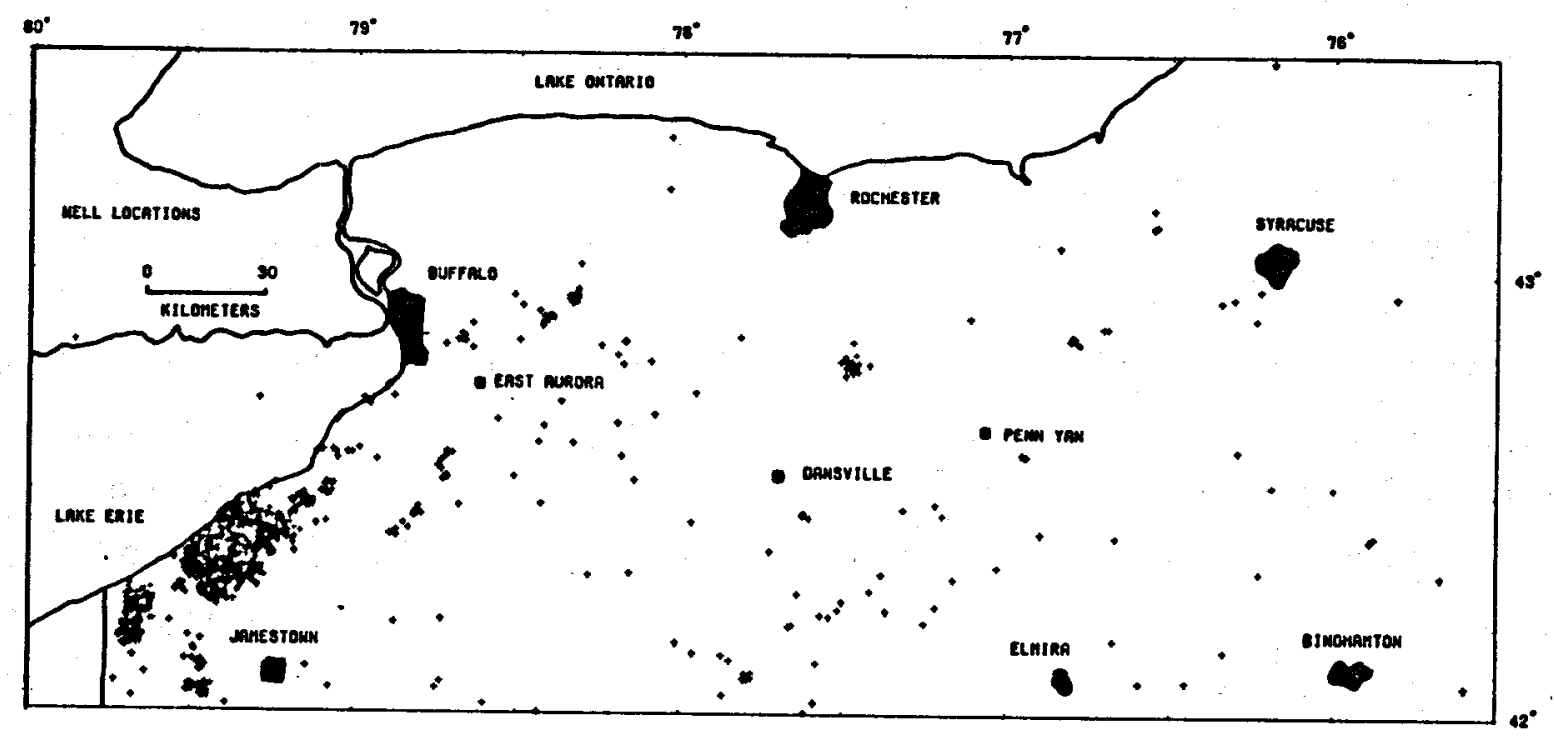

Fig. 4.

Location of wells with recorded bottom-hole temperatures.

Three prominent anomalies are evident in the map: one at East Aurora $\left(27^{\circ} \mathrm{C} / \mathrm{km}\right)$, a second east of Rochester $\left(30^{\circ} \mathrm{C} / \mathrm{km}\right)$, and a third between Elmira and Binghamton $\left(30^{\circ} \mathrm{C} / \mathrm{km}\right)$.

Our field work (see Table I) and studies by Evans and Coleman (1974) and Dowdle and Cobb (1974) document the need for a procedure that corrects bottom-hole temperature measurements for the thermal effects of drilling

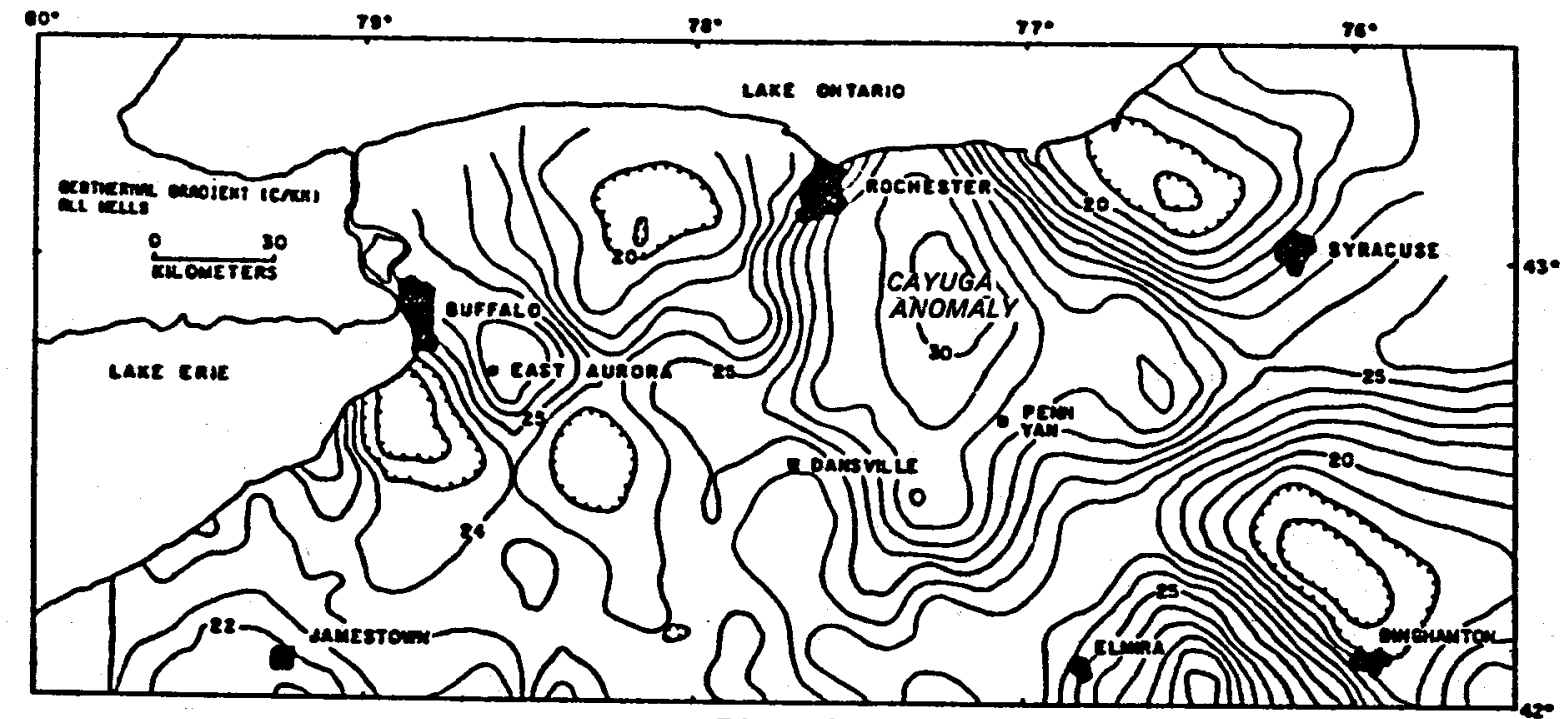

Fig. 5 .

Contoured temperature gradients for wells deeper than $500 \mathrm{~m}$ assuming no drilling disturbance correction. 
TABLE I

TEMPERATURES MEASURED IN WELL 14324, AUGUST 1, 1979

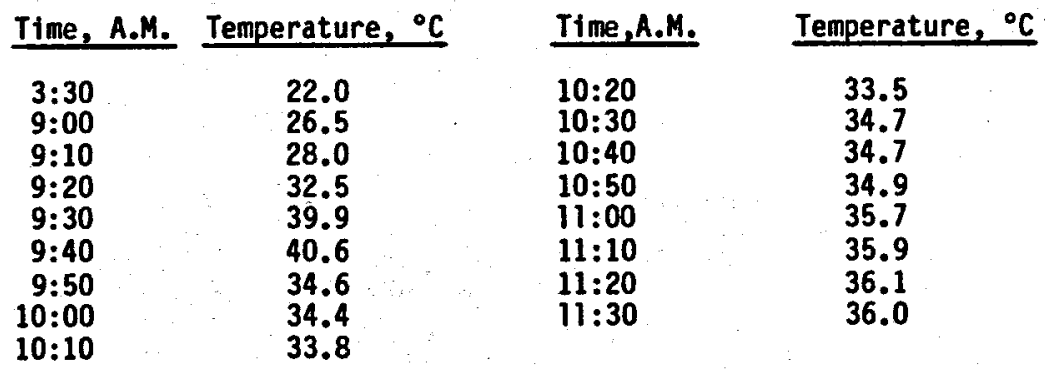

drilling. The measured bottom-hole temperatures may be significantly lower than the undisturbed equilibrium temperatures in the host rock if the temperature of the drilling medium (fluid or air) is lower than the in situ values.

Our investigations of drilling disturbances in the oil and gas wells in New York State have not been completed. Consequently, a correction was developed from the 1971 study by the AAPG, and the technique was used to correct the bottom-hole temperatures herein discussed. The correction increased temperatures in boreholes that are deeper than $75 \mathrm{~m}$, where the correction is $0^{\circ} \mathrm{C}$. At a depth of $1830 \mathrm{~m}$, for example, the corrected temperatures are $12.5^{\circ} \mathrm{C}$ higher than the bottom-hole values that were measured shortly after drilling was terminated.

Figure 6 is a contour map of the gradients that were calculated using "corrected" bottom-hole temperatures in all 837 oil and gas wells. The gradients in the data set ranged from $15.52^{\circ} \mathrm{C} / \mathrm{km}$ to $47.28^{\circ} \mathrm{C} / \mathrm{km}$. Gradient values in the computed grid matrix ranged from $21.27^{\circ} \mathrm{C} / \mathrm{km}$ to $39.09^{\circ} \mathrm{C} / \mathrm{km}$, and arithmetic grid smoothing altered the range to $22.31^{\circ} \mathrm{C} / \mathrm{km}$ to $37.57^{\circ} \mathrm{C} / \mathrm{km}$. From comparisons with Fig. 5 , it is evident that the magnitudes of the anomalies are increased when the modified AAPG correction is applied. Another prominent feature of the corrected gradient map is the short lateral increase in the temperature gradients trending north-south near Buffalo (Fig. 6). Inasmuch as some of the shallow wells $(<500 \mathrm{~m})$ in this area have anomalously high recorded temperatures, these elevated gradients probably reflect nonconductive temperatures of relatively shallow ground-water circulation systems in the underlying rocks.

To obtain a map of "conductive" corrected gradients, data for wells shallower than $500 \mathrm{~m}$ were neglected and the remaining 789 control points were 


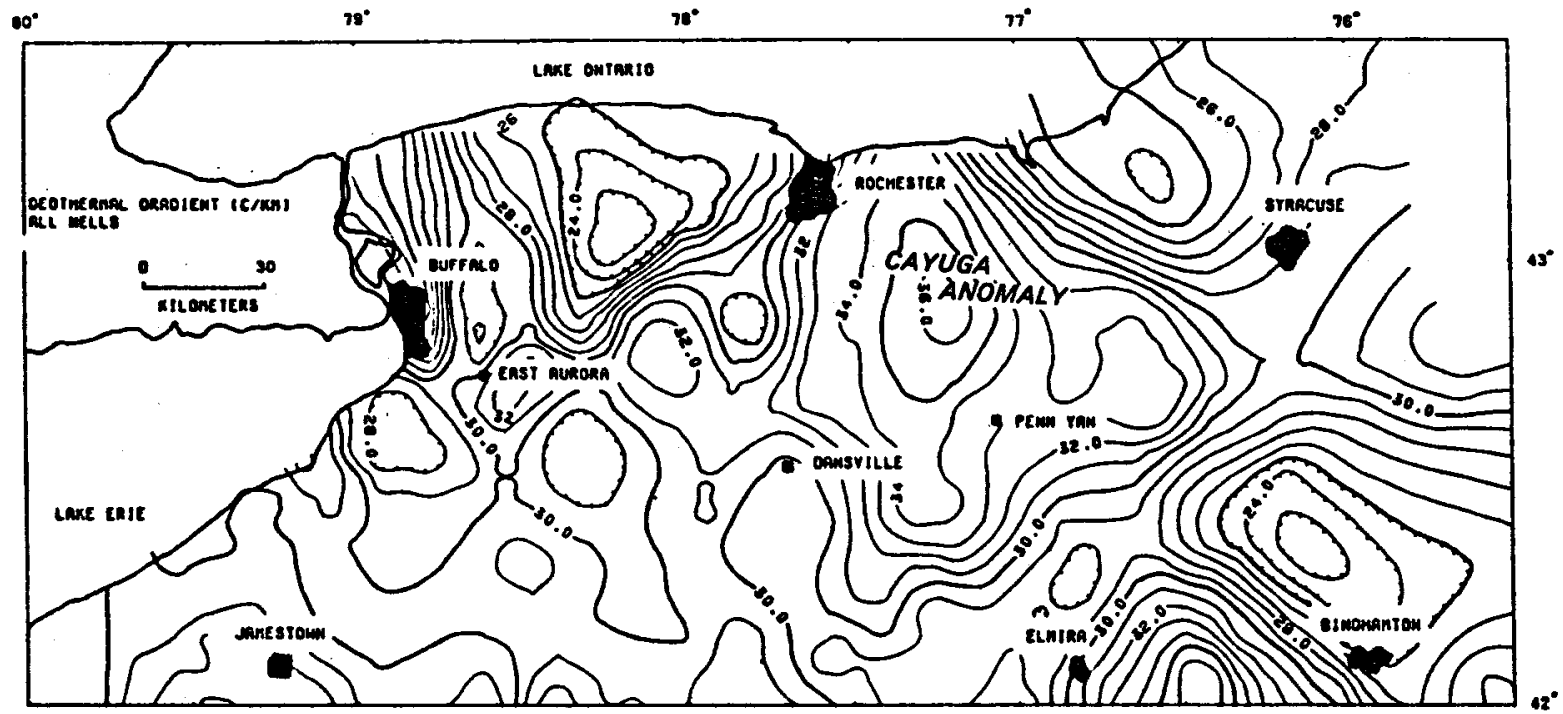

Fig. 6.

Contoured temperature gradients for all wells assuming drilling disturbance correction similar to correction used for the AAPG geothermal gradient map.

contoured. The resulting map is shown in Fig. 7. The range of geothermal in the data set for this figure is from $15.52^{\circ} \mathrm{C} / \mathrm{km}$ to $47.28^{\circ} \mathrm{C} / \mathrm{km}$. In the computed grid matrix, values for gradients ranged from $21.48^{\circ} \mathrm{C} / \mathrm{km}$ to $38.85^{\circ} \mathrm{C} / \mathrm{km}$. The grid-smoothing process reduced the range of values to $22.58^{\circ} \mathrm{C} / \mathrm{km}$ to $36.63^{\circ} \mathrm{C} / \mathrm{km}$. The contoured gradients for the East Aurora, Cayuga, and Elmira anomalies are 32,36 , and $36^{\circ} \mathrm{C} / \mathrm{km}$, respectively (Fig. 7 ).

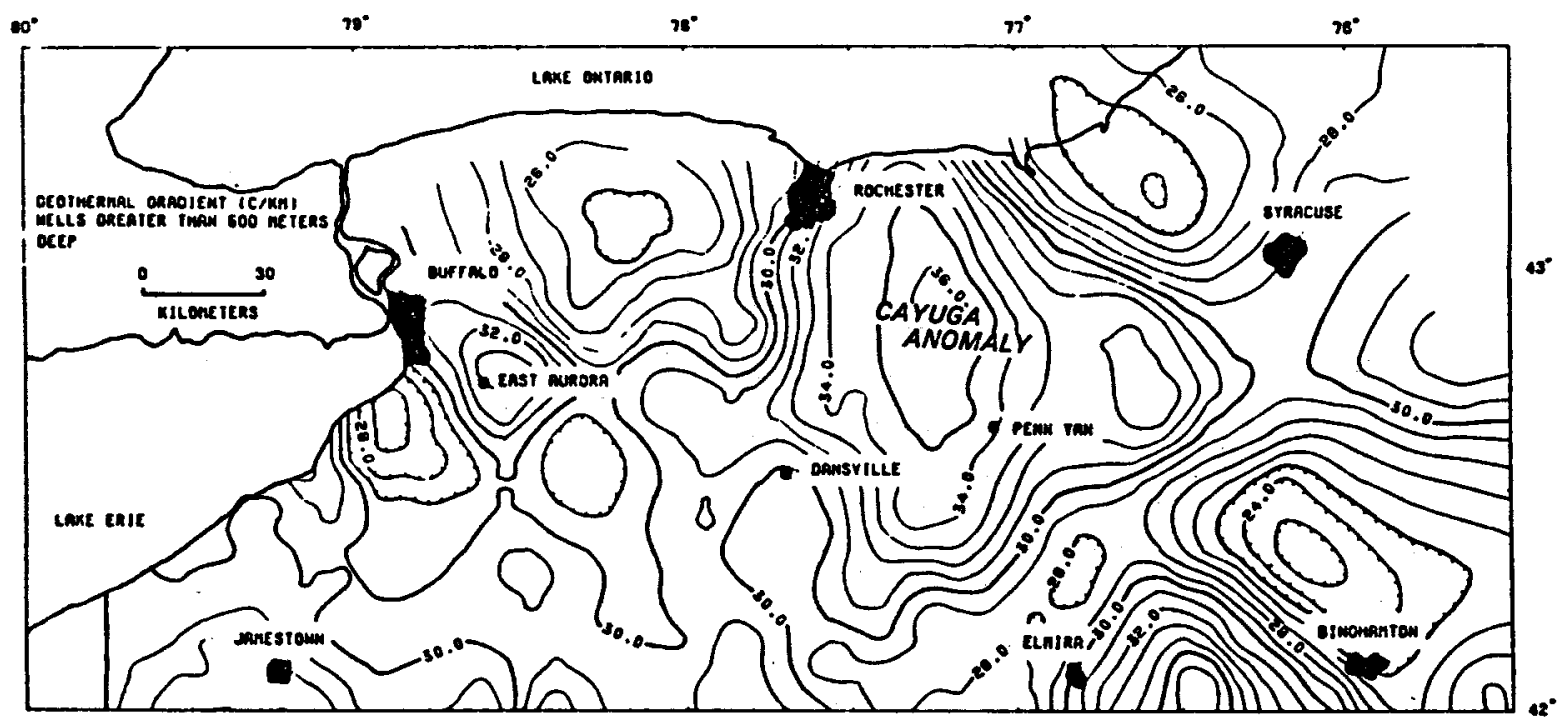

Fig. 7.

Contoured temperature gradients for all wells deeper than $500 \mathrm{~m}$ assuming drilling disturbance correction as in Fig. 6. 
Figure 8 shows a perspective diagram of the elevated temperatures highlighting the relative magnitude of the gradients. These gradients are considered maxima because the true gradients probably are between the corrected (Fig. 7) and uncorrected (Fig. 5) values.

Figure 9 is a gradient map that is based on data for 640 wells that are deeper than $750 \mathrm{~m}$. The gradients in the data set are in the range $21.28^{\circ} \mathrm{c} / \mathrm{km}$ to $45.06^{\circ} \mathrm{C} / \mathrm{km}$. Gridding created gradients in the range $22.96^{\circ} \mathrm{C} / \mathrm{km}$ to $38.32^{\circ} \mathrm{C} / \mathrm{km}$, and smoothing of the grids altered the range to $23.71^{\circ} \mathrm{C} / \mathrm{km}$ to $36.06^{\circ} \mathrm{C} / \mathrm{km}$. The Elmira and Cayuga anomalies are evident in this map, but the East Aurora anomaly has been eliminated. Figure 9 also shows a single low-gradient zone in the vicinity of East Aurora, whereas Fig. 7 shows two regions with lower gradients in this area.

Comparisons of the revised temperature gradient map (Fig. 7) and the AAPG gradient map reveal that both show a positive anomaly near East Aurora. The gradients here are greater than $32^{\circ} \mathrm{C} / \mathrm{km}$. Although both maps show a positive anomaly near Cayuga Lake, the new map is significantly different in the sense that the zone of highest gradients $\left(>36^{\circ} \mathrm{C} / \mathrm{km}\right)$ plots further to the west in an area between Rochester and Penn Yan. The new map also differs from the AAPG map in that a positive anomaly between Binghamton and Elmira has been separated from the Cayuga anomaly, and the gradients in the region are greater than $36^{\circ} \mathrm{C} / \mathrm{km}$. Our analysis of the bottom-hole temperature data from western and

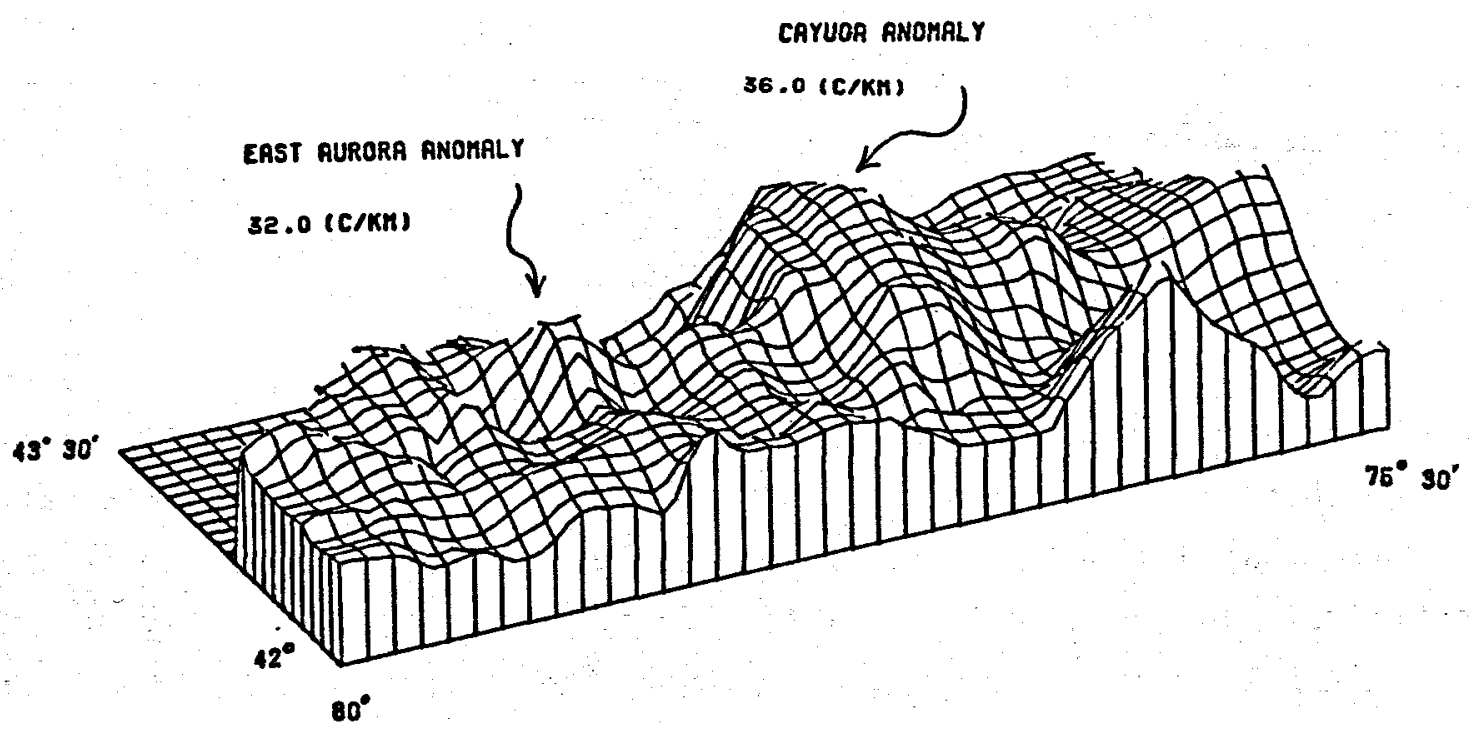

Fig. 8.

Perspective diagram of temperature gradients in New York State showing relative magnitude of the anomalies. 


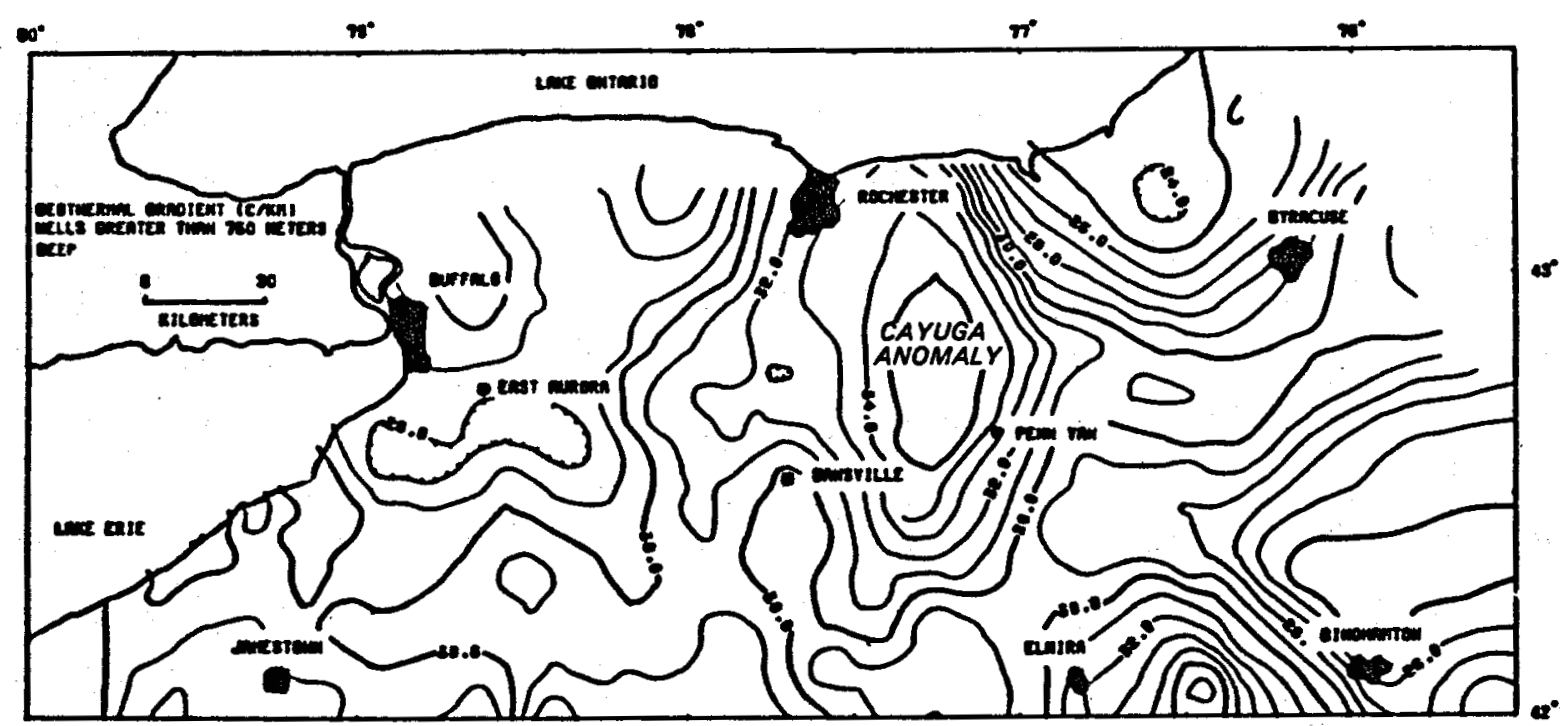

Fig. 9.

Contoured temperature gradients for wells deeper than $750 \mathrm{~m}$. A drilling disturbance correction has been applied. The East Aurora anomaly disappears because shallow data is not included.

central New York, therefore, confirms the validity of the high-gradient anomalies shown on the AAPG map, but it better defines the position of the Cayuga anomaly.

\section{DETAILED GEOTHERMAL GRADIENTS}

Reliable geothermal resource assessments require determinations of equilibrium thermal gradients in undisturbed underground openings (drill holes, tunnels, etc.). The conductive heat flow q normal to the earth's surface is calculated from the basic relation

$$
q=k \frac{d T}{d z},
$$

where $K$ is thermal conductivity of the local rock units and $d T / d z$ is the vertical geothermal gradient. In studies in sedimentary basins, it is important to show that the heat flow is vertically uniform and that there is a sympathetic relation between $K$ and $d T / d z$; for example, the highest geothermal gradients should exist in layers that have the lowest conductivity. Such analyses are important in geothermal studies in New York and Pennsylvania because the geothermal gradients within a borehole that penetrates different 
sediments should reflect the contrasting conductivities of each lithologic unit.

Equilibrium temperatures were measured in a number of wells that were drilled for purposes of extracting gas. Locations for the measured wells are listed in Table II, as are bottom-hole temperatures, average gradients, and the gradients above and below prominent formation boundaries in the holes. The gradients for the intervals above and below the indicated contacts were estimated from linear portions of the temperature-depth profiles for the holes. The temperature data and the temperature profiles for the wells are listed and plotted in Appendix $B$.

The temperature-depth logs for the wells southwest of the East Aurora anomaly (13000, 14269, 14270) show identical gradients throughout the same stratigraphic depth interval (see for example, Fig. 10, Table II, and Appendix B.). A change in the slopes of the profiles occurs at depths that correspond to the top of the Onondaga formation. In the sediments above the Onondaga, the gradient is about $36^{\circ} \mathrm{C} / \mathrm{km}$. Below this unit the average geothermal gradient decreases to about $16^{\circ} \mathrm{C} / \mathrm{km}$. When the average surface temperatures are subtracted from the bottom-hole temperatures for wells 13000,14269 , and 14270, the average gradients are calculated to be $25.7,23.6$, and $20 .{ }^{\circ} \mathrm{C} / \mathrm{km}$, respectively. These gradients agree with those on the map in Fig. 7.

Well 14324 is near the edge of the East Aurora anomaly. The relatively linear portions of the temperature profile for the hole suggest that the

TABLE II

DATA ON WELLS LOGGED IN JULY, AUGUST, OR SEPTEMBER, 1979

\begin{tabular}{|c|c|c|c|c|c|c|c|c|}
\hline Mell No. & Latitude & Longitude & $\begin{array}{l}\text { Bottom-hole } \\
\text { Temp., }{ }^{\circ} \mathrm{C}\end{array}$ & $\begin{array}{c}\text { Well depth } \\
\text { meters }\end{array}$ & $\begin{array}{l}\text { Average } \\
\text { surface } \\
\text { temp.. }\end{array}$ & $\begin{array}{l}\text { Average } \\
\text { Gradient } \\
{ }^{\circ} \mathrm{C} / \mathrm{km}\end{array}$ & $\begin{array}{c}\text { Gradient above } \\
\text { Onondaga Ls } \\
{ }^{\circ} \mathrm{C} / \mathrm{km}\end{array}$ & $\begin{array}{c}\text { Gradient below } \\
\text { Onondaga Ls } \\
{ }^{\circ} \mathrm{C} / \mathrm{km}\end{array}$ \\
\hline $\begin{array}{l}13738 \\
13000 \\
14269 \\
14270 \\
14310 \\
14324 \\
14365 \\
14172 \\
14423\end{array}$ & $\begin{array}{lll}42^{\circ} & 52^{\prime} & 25^{\prime \prime} \\
42^{\circ} & 37^{\prime} & 49^{\prime \prime} \\
42^{\circ} & 39^{\circ} & 29^{\prime \prime} \\
42^{\circ} & 38^{\prime} & 45^{\prime \prime} \\
42^{\circ} & 03^{\prime} & 34^{\prime \prime} \\
42^{\circ} & 43^{\prime} & 53^{\prime \prime} \\
42^{\circ} & 04^{\prime} & 47^{\prime \prime} \\
42^{\circ} & 47^{\prime} & 57^{\prime \prime} \\
42^{\circ} & 45^{\circ} & 45^{\prime \prime}\end{array}$ & $\begin{array}{lll}76^{\circ} & 40^{\prime} & 21^{\prime \prime} \\
79^{\circ} & 00^{\prime} & 23^{\prime \prime} \\
78^{\circ} & 55^{\prime} & 29^{\prime \prime} \\
78^{\circ} & 56^{\prime} & 09^{\prime \prime} \\
79^{\circ} & 18^{\prime} & 28^{\prime \prime} \\
78^{\circ} & 37^{\prime} & 25^{\prime \prime} \\
79^{\circ} & 21^{\prime} & 13^{\prime \prime} \\
77^{\circ} & 27^{\prime} & 45^{\prime \prime} \\
78^{\circ} & 40^{\prime} & 10^{\prime \prime}\end{array}$ & $\begin{array}{l}27.69 \\
24.86 \\
22.45 \\
22.9 \\
38.9 \\
28.7 \\
38.9 \\
18.72 \\
22.57\end{array}$ & $\begin{array}{r}573.0 \\
618.0 \\
566.9 \\
686.1 \\
1310.6 \\
640.1 \\
1249.7 \\
426.7 \\
548.6\end{array}$ & $\begin{array}{l}9.48 \\
8.9 \\
9.1 \\
9.2 \\
8.29 \\
7.84 \\
8.59 \\
6.73 \\
8.269\end{array}$ & $\begin{array}{l}31.78^{+} \\
25.7^{2} \\
23.6 \\
20.09 \\
23.36 \\
32.6 \\
24.26 \\
28.09 \\
26.067\end{array}$ & $\begin{array}{l}35.01 \\
31.55 \\
33.71 \\
27.36 \\
38.87 \\
18.93 \\
23.75 \\
37.45\end{array}$ & $\begin{array}{l}39.95 \\
17.72 \\
16.85 \\
14.50 \\
16.84 \\
19.94 \\
5.9 \\
22.57 \\
17.70\end{array}$ \\
\hline
\end{tabular}

+Average gradient directly follows bottom-hole temperature in Queenston Shale and the calculated surface temperature. 


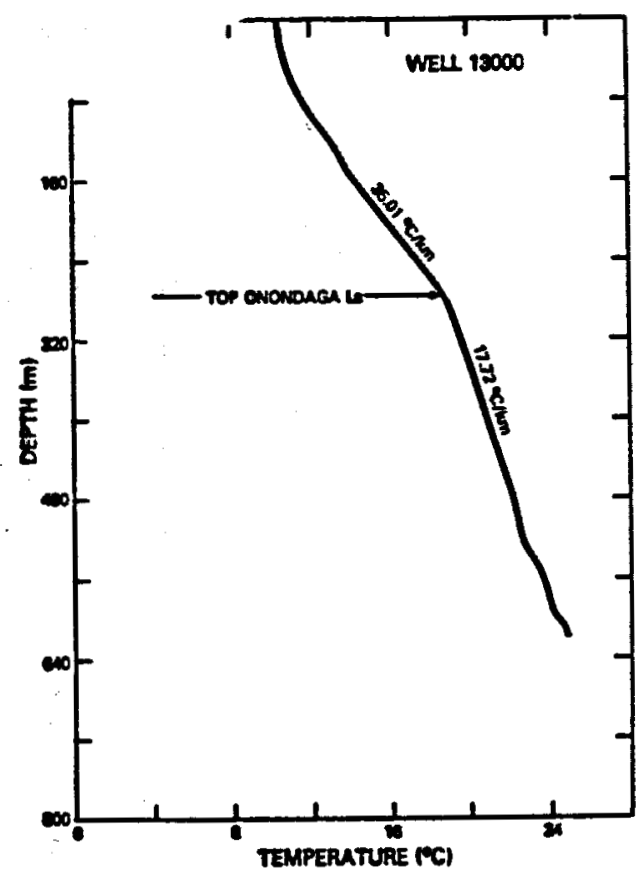

Fig. 10.

Temperature-depth profile for well 13000 showing different gradients above and below the top of the Onondaga Limestone. well was close to therimu.

rium when measurements were man

The calculated gradient above the Onondaga is $38.87^{\circ} \mathrm{C} / \mathrm{km}$ and that for the units below is $19.94^{\circ} \mathrm{C} / \mathrm{km}$. The average geothermal gradient for the hole is $32.6^{\circ} \mathrm{C} / \mathrm{km}$, a value close to that shown for the area in the contour map in Fig. 6 .

The temperature profiles for the wells in the southwest section of New York State exhibit similar variations in geothermal gradients. The distinct change in the slope of the profiles occurs at a greater. depth in wells 14310 and 14365 than it does in the East Aurora wells. The gradient in the upper section of 14310 is $27.4^{\circ} \mathrm{C} / \mathrm{km}$, and the lower section has a gradient of $16.8^{\circ} \mathrm{C} / \mathrm{km}$.

Like the profiles for the wells near East Aurora, the stratigraphic location of the change of slope corresponds to the top of the Onondaga limestone.

The relatively higher gradients in the shallower parts of the abovediscussed wells occur in sections of Devonian shale. These data suggest that these shales have a low conductivity and the corresponding gradient is high.

Thermal disequilibrium and/or disturbances in a drill hole can be caused by water circulation, thermal effects of drilling, natural gas expulsion, and chemical reactions. Some of our temperature measurements were made in wells that were not cased to total depth, so water circulation may have occurred near the bottoms of these holes. In wells 13738 and 14172 apparent water circulation caused a depression in the measured geothermal gradients. In well 14172 the temperatures were logged within minutes of the time that stored gas was released from the hole. When gas was released, the ambient pressure dropped from 40 psi to 0 psi in about 5 minutes of expulsion. Because gas cools rapidly during expansion and release to the surface, the measured 
temperatures in this well may be lower than the undisturbed ambient values and may not indicate the equilibrium geothermal gradients.

Most of the gas wells in New York State are drilled with air percussion rigs, and the transient thermal effects of air percussion drilling are not accurately known for this study area.* To evaluate this effect and estimate a correction for bottom-hole temperatures, measurements were made in one well for a period of several hours after drilling was terminated. The results show that the bottom-hole temperature increased from $22.0^{\circ} \mathrm{C}$ to $36^{\circ} \mathrm{C}$ over 8.0 hours (Table I). This isolated experiment precludes quantitative evaluation of the effect of drilling, but the time-dependent change of the bottom-hole temperature is consistent with results reported by other authors (for example, Middleton 1974).

\section{THERMAL CONDUCTIVITY OF SEDIMENTS IN NEW YORK}

The lateral and vertical variations of conductivity must be known if bottom-hole and other temperature data are to be utilized to produce detailed heat flow and subsurface temperature maps. Thus structure contour maps of the Lower Devonian Onondaga Limestone, the Upper Ordovician Queenston Shale, and the Precambrian basement were prepared.

Data were taken from Kreidler et al. (1972), Kreidler (1975), and drillers' logs obtained from the New York State Geological Survey. To help alleviate "edge effect" problems, data were obtained for those counties in northern Pennsylvania that border New York (Commonwealth of Pennsylvania, 1960-1978). Gridding of the data was done using a specially adapted transverse mercator projection FORTRAN program. The data, except those taken directly from Kreidler's (1975) map of deep disposal wells, are listed in Appendix $C$.

The Onondaga Limestone is of particular interest because it appears to mark a distinct change of subsurface geothermal gradients. Additionally, the Onondaga is overlain by several hundred meters of Upper Devonian black shales (Richard, 1975). This sharp contrast in lithology is usually noted in driller logs and so it provides an excellent stratigraphic marker. Two hundred thirty data points of sub-sea-level depths of the Onondaga (Appendix $C$ ) were machinecontoured using the Surface II Graphics System (Sampson, 1975) (Fig. 11). A

*Bottom-hole temperatures are normally measured about 4-12 hours after drilling is stopped in potential gas wells. 
sixth-order trend surface polynomial was also formulated. The results permit reasonably accurate prediction of the depth to the Onondaga at any arbitrary location within western and central New York State.

The Queenston Shale is also of considerable interest to the natural gas industry of New York, and, as a result, it is frequently noted in drilling logs; its distinctive red coloration makes accurate identification probable. The large number of depth control points (190) and the fact that the Queenston is in the middle of the sedimentary section (Fisher, 1977) makes it a good second choice for a marker horizon (Fig. 12).

Basement structure also must be known for reliable interpretation of heat flow and other geophysical studies. In addition, determinations of thickness of sediments above a postulated abnormal source of heat in the basement are essential for estimation of the subsurface temperatures. Inasmuch as some high geothermal gradients in New York State correlate with areas of anomalously low Bouguer gravity, it is necessary to determine whether the gravity anomalies are due to lateral compositional variation (and perhaps truly related to heat flow), or whether these gravity anomalies can be attributed to basement topography. Accurate determination of basement structure requires adequate control. Because sedimentary cover thickens markedly to the south in New York State (Fisher, 1977); at least $50 \%$ of all control wells are located within $50 \mathrm{~km}$ of the shore of Lake Ontario. Therefore, control was judged

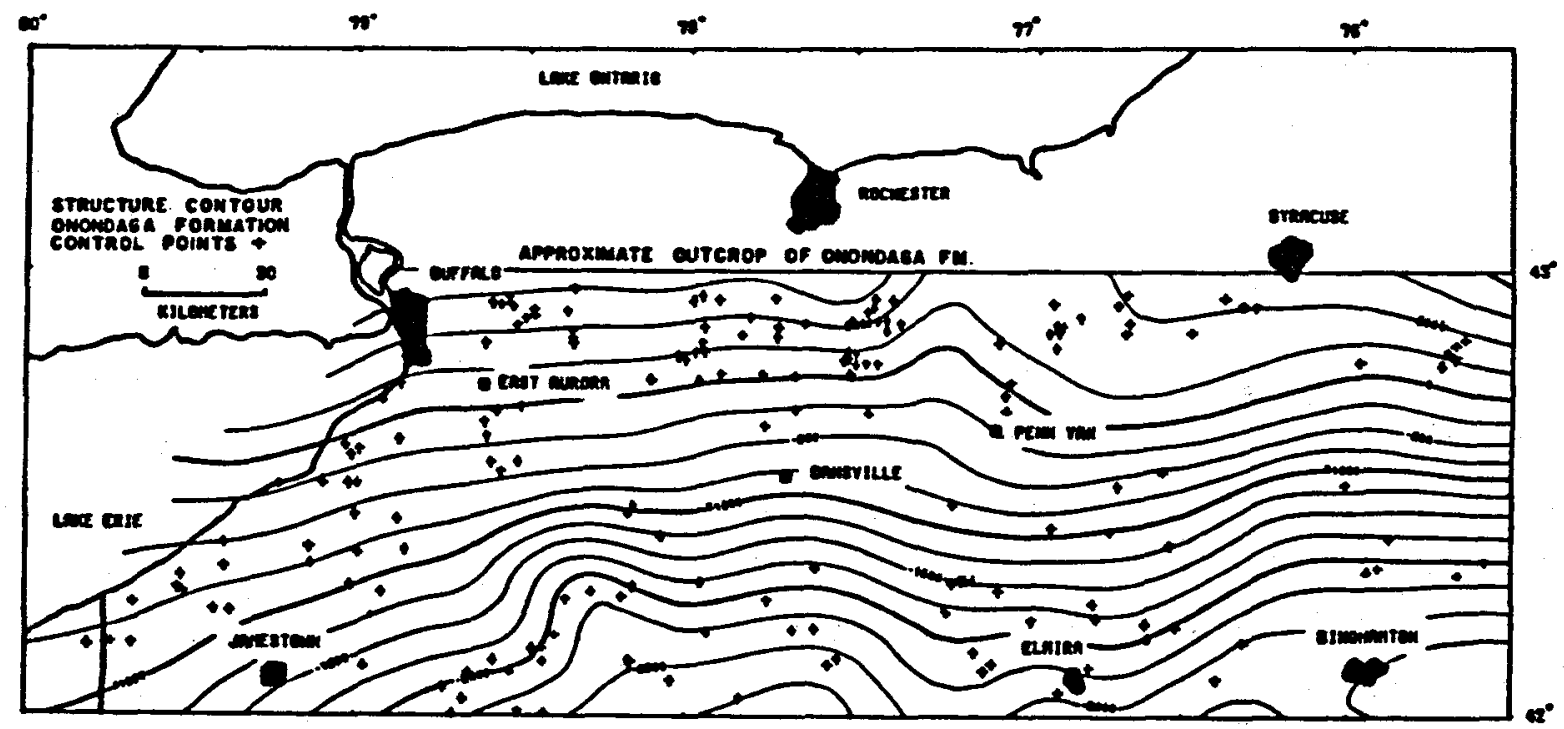

Fig. 11 .

Depth below sea level to the top of the Onondaga Formation. Devonian black shales occur above this contact. 


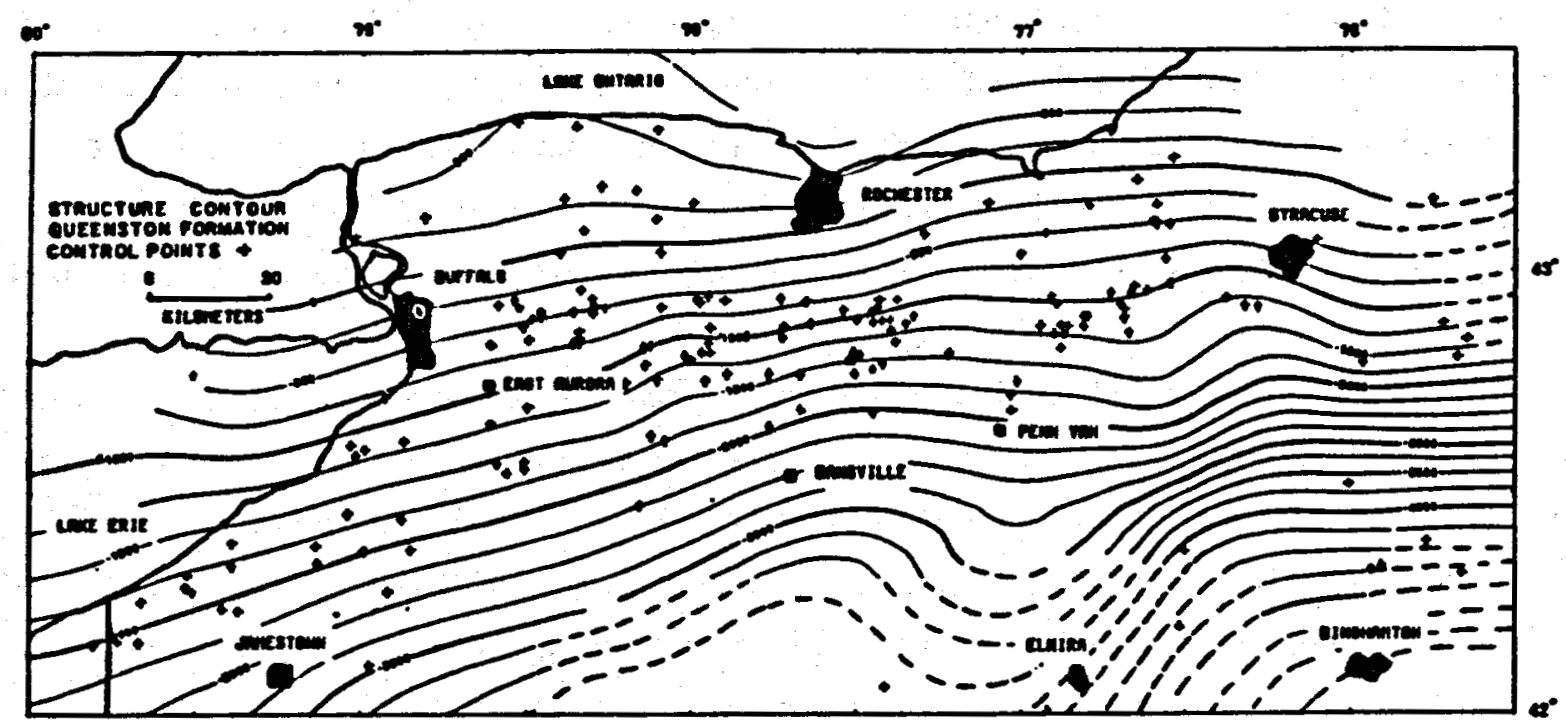

Fig. 12.

Depth below sea level to the top of the Ordovician Queenston Formation.

insufficient for direct contouring. Instead, a third-order trend surface map was prepared using Surface II (Fig. 13). Fortunately, stratigraphic control in the northern and central parts of the Cayuga anomaly was sufficient to allow study of basement topographic trend surface residuals.

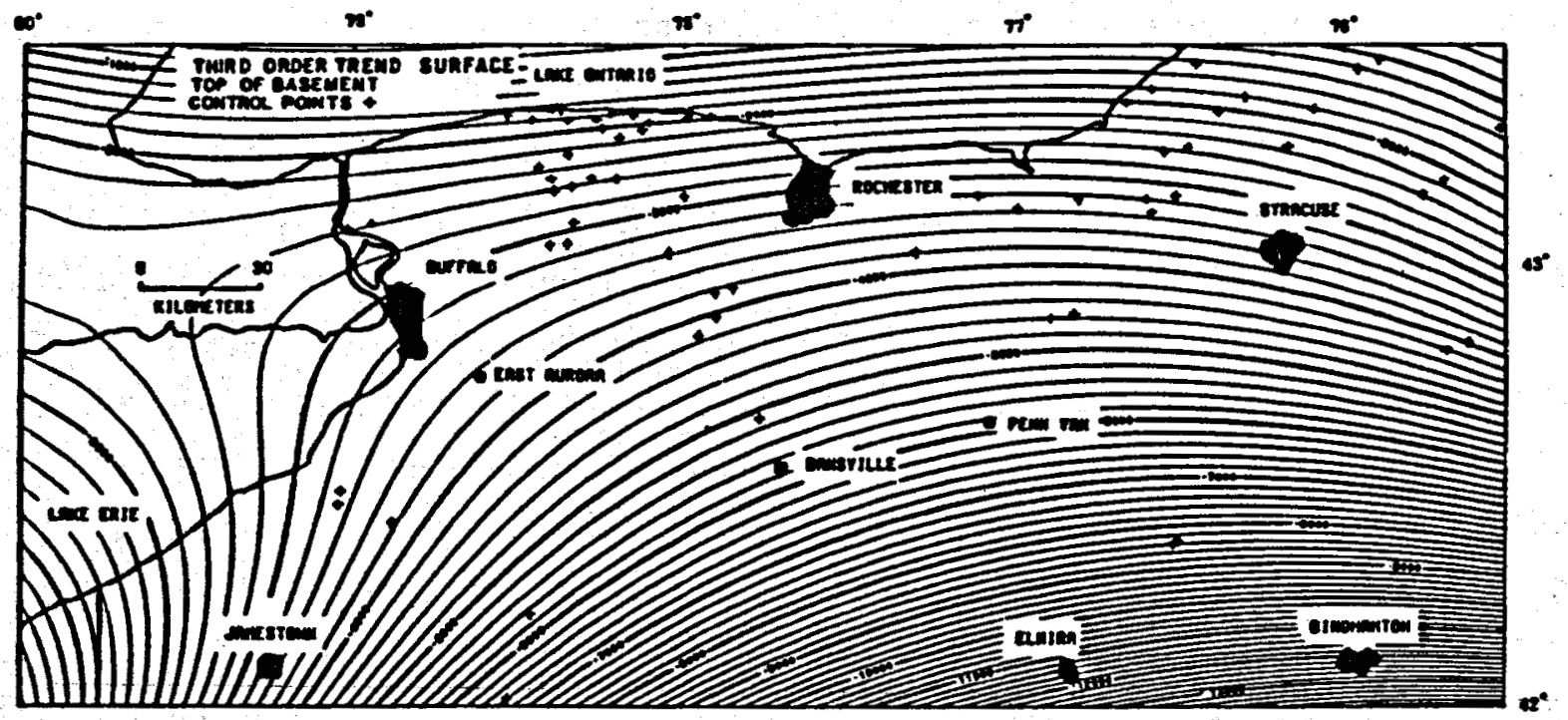

Fig. 13.

Depth below sea level to the basement. Data are approximated by a third-order trend surface. 
The structure contour map of the basement (Fig. 13) shows a gradual deepening from about $600 \mathrm{~m}$ below sea level in the extreme northwest to about $4000 \mathrm{~m}$ below sea level near Binghamton in the southeasten part of the map. Control is rather poor and the mathematical trend surface contour lines begin to show an inflection that is unjustified by the data just west of the longitude for Jamestown.

In Fig. 11 the Onondaga Limestone is shown to dip gradually to the south; the elevation of the upper contact changes from about $155 \mathrm{~m}$ above sea level at the outcrop in the north to about $770 \mathrm{~m}$ below sea level in the south. Figure 12 shows a similar trend for the Queenston Shale, which is about $325 \mathrm{~m}$ lower in the stratigraphic column. Control is very good for the Onondaga and, checks of well data against the map show excellent correlation.

Spot checks of original well data around the northern and north-central parts of the Cayuga anomaly indicate no variation greater than $40 \mathrm{~m}$ from the known basement elevation (Fig. 13) for the mathematical trend surface calculated over an area at least twice as wide as the anomaly itself. There is no reason at the present time to believe that basement topography bears any relation to the gravity low in this region (see below). It is much more reasonable to conclude that a lateral compositional contrast in the Precambrian is responsible.

Detailed temperature logs for several wells show excellent agreement between predicted depth to the Onondaga Limestone and the depth to a prominent change of temperature gradient. This change always occurs as an abrupt decrease from a higher gradient to a lower gradient as depth increases; this implies an abrupt increase in conductivity below the Upper Devonian Marcellus Shale-Onondaga Limestone contact. Joyner (1960) also noted contrasting gradients in the "Marcellus type" Upper Devonian Shales and the Onondaga Limestone in West Virginia and Pennsylvania and inferred that they were related to the thermal conductivities of the sediments. Our data and that of Joyner suggest that the lithologic units in New York State can be separated into distinct conductivity units (see Table III). The structural contour maps of the basement,. Onondaga, and Queenston horizons therefore allow a threedimensional representation of the conductivity variation throughout the State. This conductivity variation could be combined with bottom-hole temperatures to produce a map of heat flow. The heat flow and conductivity maps could then be used to predict subsurface temperatures throughout the region. 
TABLE III

THERMAL CONDUCTIVITIES OF SAMPLES FROM WELLS IN NEW YORK, PENNSYLVANIA, AND WEST VIRGINIA

\begin{tabular}{lccc} 
Category & $\begin{array}{c}\text { No. } \\
\text { Samples }\end{array}$ & $\begin{array}{c}\text { Average Conductivity } \\
\text { at } 30^{\circ} \mathrm{C} \text { in } \mathrm{W} /{ }^{\circ} \mathrm{C} \mathrm{m}\end{array}$ & $\begin{array}{c}\text { Range of Conductivity } \\
\text { in } \mathrm{W} /{ }^{\circ} \mathrm{C} \mathrm{m}\end{array}$ \\
\hline & & & \\
Quartzose Sandstones & 7 & 4.40 & $3.35-5.23$ \\
Impure Sandstones & 5 & 2.67 & $1.88-3.35$ \\
Sandy Sandstones & 3 & 1.98 & $1.61-2.21$ \\
Shales & 5 & 1.41 & $1.26-1.51$ \\
Limestones & 3 & 2.58 & $2.23-2.91$ \\
Calcareous Shales & 2 & 1.89 & $1.76-2.05$ \\
\hline
\end{tabular}

Adapted from Joyner (1960, Table, p. 1232).

VI. GEOCHEMISTRY

Samples of ground water from the Cayuga and East Aurora anomalies and adjacent areas were collected and analyzed to determine if there was chemical evidence for local hydrothermal activity. The analyses were performed by standard procedures and results are listed in Appendix $D$.

Qualitative and quantitative geothermometry. $\left(\mathrm{SiO}_{2}, \mathrm{NaKCa}\right.$, and $\mathrm{NaKCaMg}$ geothermometers) was applied to the analyses and the results were compared to the geophysical interpretations. None of the samples showed clear evidence of having originated within an active hydrothermal system. More encouraging results, however, were obtained by using the silica-heat flow method of Swanberg and Morgan (1979).

The silica geothermometer is based on the temperature dependence of quartz solubility in water. The procedure can be used to estimate the last temperature of water-rock equilibrium (Fournier and Rowe, 1966; Fournier et al., 1974) and to estimate regional heat flow (Swanberg and Morgan, 1979). The following equations can be used to convert ground-water silica content (ppm) to geotemperature and heat flow.

and

$$
\mathrm{T} \mathrm{SiO} 2=\frac{1913}{5.205-\log _{10} \mathrm{SiO}_{2}}-273.15
$$

$$
\mathrm{T} \mathrm{SiO}_{2}=.67 q+13.2
$$

where $\mathrm{T} \mathrm{SiO}_{2}$ is the silica geotemperature in ${ }^{\circ} \mathrm{C}$ and $\mathrm{q}$ is heat flow in $\mathrm{mW} / \mathrm{m}^{-2}$. 
Figure 14 is a histogram of heat flow values obtained by applying the silica method. The mean regional flux predicted for the study area is 41.4 $\mathrm{mWm}^{-2}$, a value that is tectonically reasonable and consistent with the values obtained by traditional heat flow techniques (Diment et al., 1972). Further, an attempt was made to isolate the higher silica-heat flow values to determine if their areal distribution would delineate the Cayuga and East Aurora anomalies in a manner similar to the gravity and bottom-hole temperature data. Figure 15 shows the areal distribution of the higher silica-heat flow values $\left(>45 \mathrm{mwm}^{-2}\right)$ and the rest of the data set. This approach appears to work

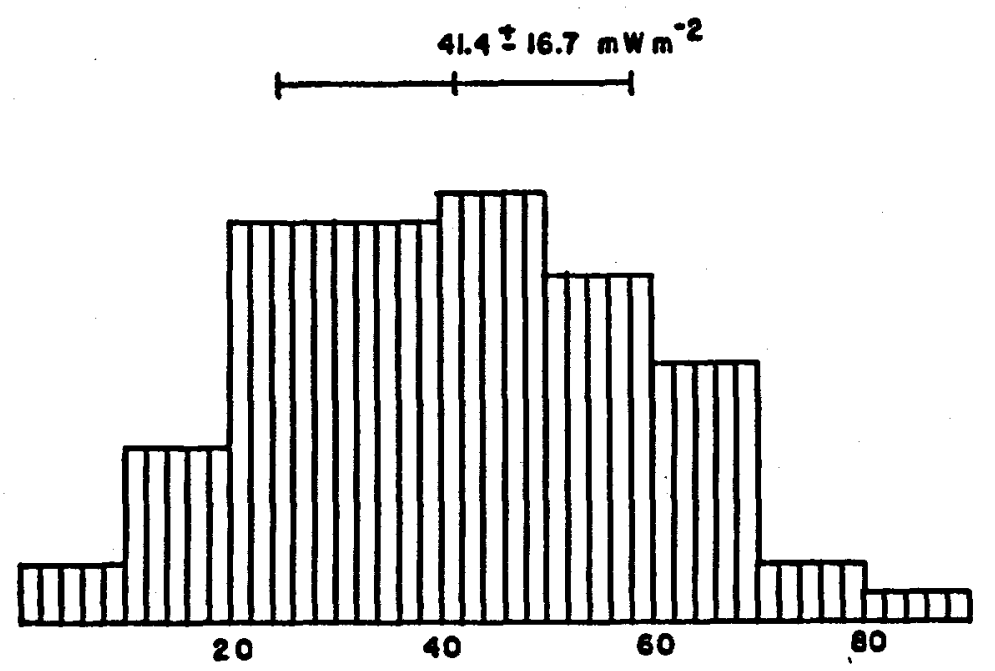

Fig. 14 .

Frequency histogram of heat flow values determined using silica geothermometry.

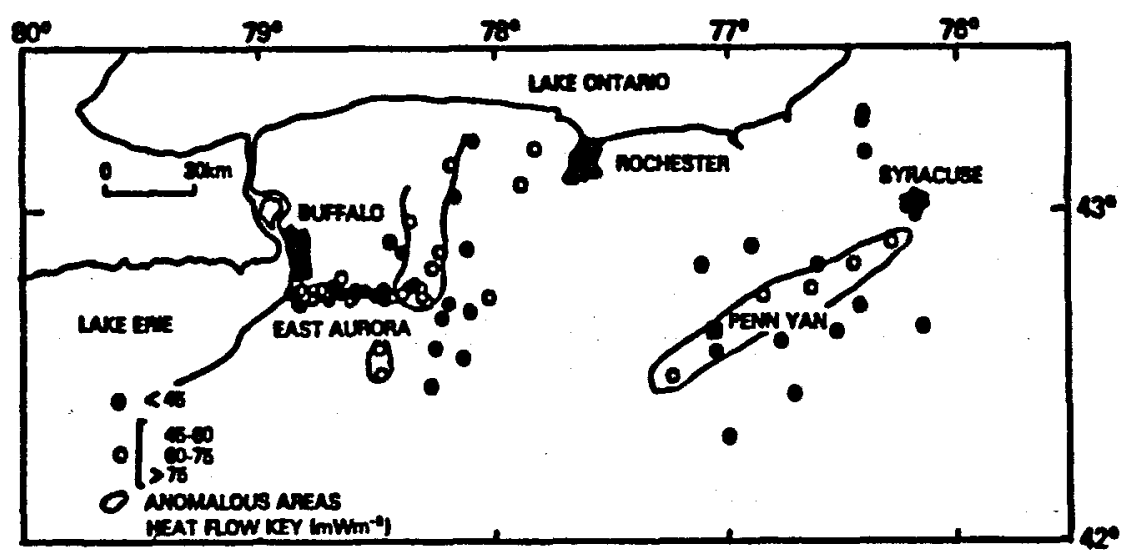

Fig. 15.

Map showing heat flow values determined using silica geothermometry. 
reasonably well for the Cayuga anomaly. The higher values plot along a line trending northeast-southwest through an area which includes the Cayuga anomaly as defined by the bottom-hole temperature data (Fig. 6), the gravity data (Fig. 16), and the previously published temperature gradient map of North America (AAPG, 1976).

The silica technique is only partially successful for the East Aurora anomaly. As shown in Fig. 15, the higher heat flow values tend to cluster in several discrete groups in an area that includes the East Aurora anomaly, but the pattern is different from those implied by the gravity and bottom-hole temperature data (Figs. 7 and 16). Part of this difference probably results from the nature of the ground waters available for study. At the Cayuga anomaly, the samples were collected from wells that penetrated the older sediments, so they may represent "old" meteoric waters that have circulated in the sedimentary section for a period of time long enough to permit chemical equilibrium with the host rock. Such equilibrium is a fundamental requirement of chemical geothermometry. On the other hand, some of the samples from the East Aurora area were from wells that penetrated glacial tills in topographic lows. Such waters may reflect "new" meteoric waters that have not had sufficient time in the ground to achieve chemical equilibrium. From the silica data, both geothermal areas have heat flows in the range $50-70 \mathrm{mWm}^{-2}$.

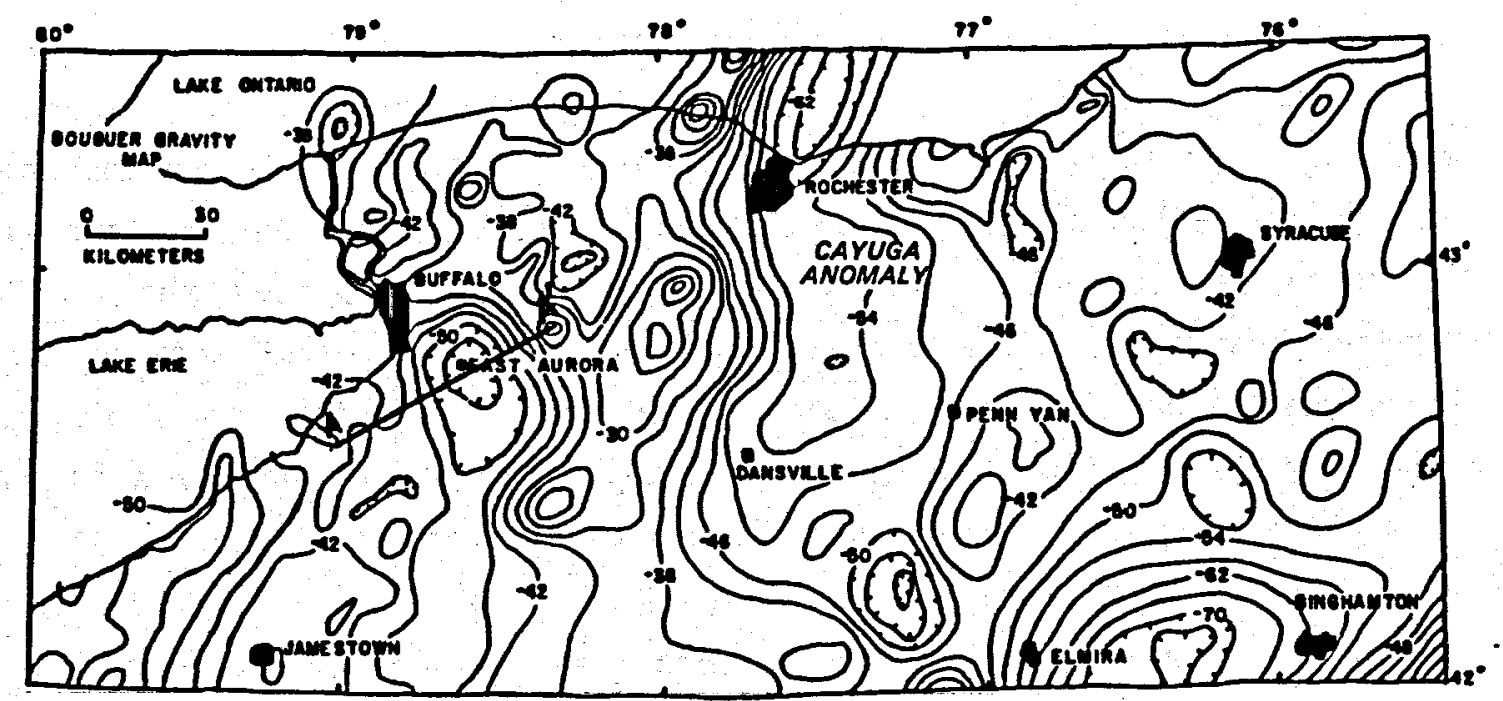

Fig. 16.

Bouguer gravity map of central and western New York State (after Revetta and Diment, 1971). 
VII. BOUGUER GRAVITY OF CENTRAL AND WESTERN NEW YORK

The Bouguer gravity map of central and western New York (Fig. 16) was largely taken from the Bouguer map of New York State (Revetta and Diment, 1971). The Bouguer anomaly field is characterized by two distinct zones that are separated by a north-south trending high gravity gradient area that is west of Rochester and extends as far south as Arcade, New York. This high gradient zone coincides with the Clarendon-Linden fault zone (Diment et al., 1972). The Bouguer anomaly field in the western part of the area shows distinct positive and negative closed anomalies and a prominent negative anomaly near East Aurora. (the East Aurora anomaly).

In the eastern part of the map area the anomalies are much more subdued, with few positive anomalies. A low-amplitude negative anomaly occurs about 20 $\mathrm{km}$ east of Rochester. This relative low strikes north-south and becomes a large negative anomaly over Lake Ontario. This negative anomaly (the Cayuga anomaly) coincides with a distinct temperature gradient anomaly shown in fig. 6. The Bouguer anomaly on the southeast corner of Fig. 16 decreases to -76 mgals in the area where depth to bedrock exceeds $3700 \mathrm{~m}$. A positive temperature gradient anomaly coincides with this Bouguer negative anomaly.

Because undeformed, near-horizontal sedimentary rocks are found to depths greater than $1080 \mathrm{~m}$, most of the changes in the Bouguer field reflect density differences in the Precambrian basement. Similar basement rocks crop out in southern Ontario, and the Bouguer gravity in this part of Canada shows a strong correlation with Precambrian geology. Briefly, the Bouguer anomalies over granitic rocks are typically negative, and those over gabbroic igneous rocks are strongly positive. These plutonic units are bordered by metamorphic rocks. Using the geologic relations as a general guide, the negative anomaly over East Aurora is attributed to a granitic pluton that is near the top of the Precambrian basement. For a density contrast of $-0.09 \mathrm{gm} / \mathrm{cm}^{3}$ and assuming a two-dimensional approximation, a density contrast as thick as $5 \mathrm{~km}$ may explain the East Aurora low (Fig. 17). The Cayuga negative anomaly also may be due to a granitic pluton in the basement. The gravity low here is not as distinct as that near East Aurora.

There are distinct correlations between the temperature gradient and gravity maps (Figs. 7 and 16). The East Aurora negative gravity anomaly coincides spatially with a positive temperature gradient anomaly. The positive Cayuga temperature gradient anomaly trends in a north-south direction and also 


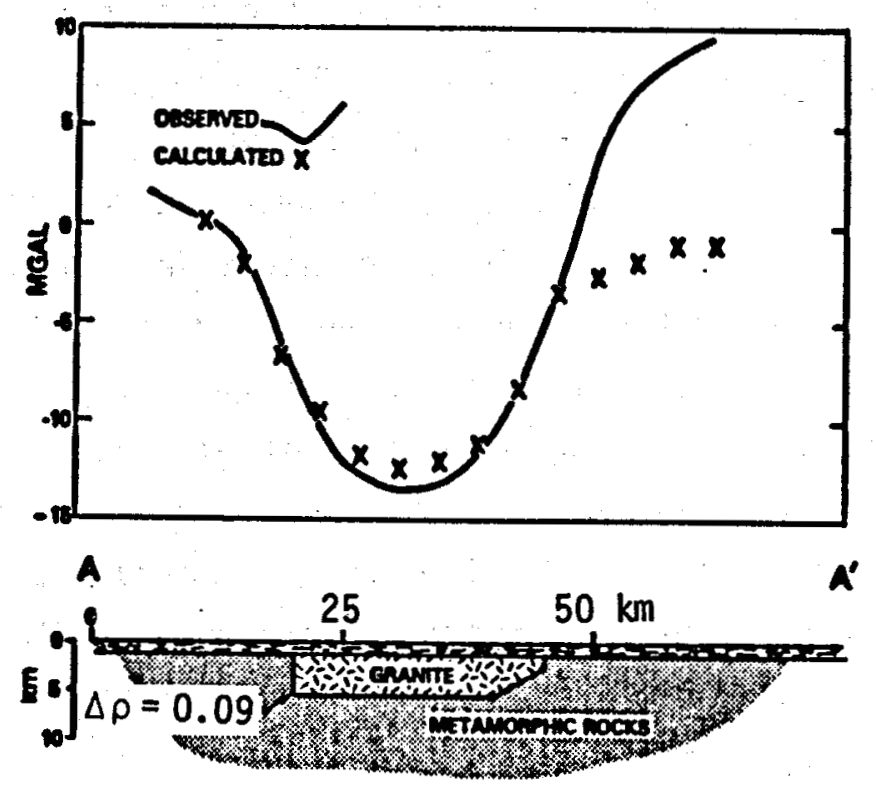

Fig. 17.

Profile and computed gravity model over the East Aurora temperature gradient anomaly.

correlates with a Bouguer gravity low. The correlations between the Bouguer gravity map and the temperature gradient map suggest that the thermal anomalies may be due to radioactive heat from Precambrian granitic rocks in the basement. Low geothermal gradients occur with low gravity values in the southeastern portion of the map. Part of this relative Bouguer low (n - 70 mgal) could be due to low densities of the underlying thick sediments.

\section{CONCLUSIONS}

Bottom-hole temperatures in a major part of New York State have been analyzed to produce two contour maps: (1) one of minimum estimated geothermal gradients, and (2) one of gradients based on temperatures corrected for the first-order effects of drilling disturbances (after AAPG, 1971). The general features of the two contour maps are similar, but the magnitudes of the gradient anomalies are significantly different. On the new map produced using the corrected temperatures, there are distinct anomalies near East Aurora, Cayuga Lake, and Elmira. Maximum temperature gradients for these anomalies are $32^{\circ} \mathrm{C} / \mathrm{km}, 36^{\circ} \mathrm{C} / \mathrm{km}$, and $36^{\circ} \mathrm{C} / \mathrm{km}$, respectively.

Equilibrium temperature measurements in a number of wells indicate that the thick Devonian black shales in New York are a low conductivity layer that has temperature gradients in the range $32^{\circ}$ to $40^{\circ} \mathrm{C} / \mathrm{km}$. The gradients are lower 
in the underlying sediments. The detailed structural contour maps presented herein may be used to estimate thermal conductivities of the sedimentary rocks and thus subsurface temperatures in the region.

There is a remarkable correlation between the spacial distribution of the high-temperature gradient anomalies and negative Bouguer gravity anomalies. Comparison of the temperature gradient and gravity maps suggests that the same subsurface bodies explain heat flow and Bouguer anomalies. A few basement samples and examinations of the gravity field in exposed areas of the Precambrian shield in Canada strongly suggest that the negative gravity anomalies are due to granitic rocks in the basement. Inasmuch as granitic rocks often contain more uranium, thorium, and potassium than mafic units, the anomalous temperature gradients may occur in sediments that overlay relatively high radioactive granites in the basement.

The preliminary results indicate that New York State has temperature gradients that are as high as any of those observed elsewhere in the eastern United States. Therefore, future analyses may define the magnitude and extent of the existing and new geothermal anomalies.

\section{ACKNOWLEDGMENTS}

We wish to thank the numerous companies and individuals that provided access and background information on gas wells that were used for temperature measurements. These include: Bounty 0i1 Co., George Stoiber; Environmental Gas, John Swanson; Connecticut Gas, Fred Stead; Sinclairville Petroleum, Georgio Pannella; and Inland Drilling Co., Inc. The cooperation of the NYS Geological Survey, $0 i 1$ and Gas Section, Dr. Arthur Van Tyne, is appreciated. Dave Borden analyzed many of the water samples, and T. T. Kittle provided valuable assistance on much of the computing. Ed Decker, Los Alamos National Laboratory, compiled and edited the manuscript. This work was sponsored by US Dept. of Energy, Division of Geothermal Energy as part of the National Hot Dry Rock Program.

\section{REFERENCES}

A.A.P.G.-U.S.G.S., "Subsurface Temperature Map of North America," U.S. Geol. Surv. Map, Arlington, Virginia (1976).

A.A.P.G., "Geothermal Survey of North America; 1971 Annual Progress Report," R. 0. Kehle, Chairman, University of Texas, Austin, Texas (1971). 
Commonwealth of Pennsylvania, Bureau of Topographic and Geologic Survey, Summarized Records of Deep Wells, 1960-1978 editions, inclusive (Harrisburg, Pennsyivania).

Diment, W. H., T. C. Urban, and F. A. Revetta, "Some Geophysical Anomalies in Eastern United States," in The Nature of the Solid Earth (McGraw Hill, New York, 1972), 544-572.

Dowdle, W. and W. Cobb, "Estimation of Static Formation Temperatures From Well Logs," Society of Petroleum Engineers, American Institute of Mechanical Engineers Paper SPE 5036 (1974).

Evans, T. and N. Coleman, "North Sea Geothermal Gradients," Nature 247, 28-30 (1974).

Fisher, Donald W., "Correlation of the Hadrynian, Cambrian and Ordovician Rocks in NYS," Univ. of the State of N. Y./St. Educ. Dept. Map and Chart Series No. 25, Albany, New York (1977).

Fournier, R. 0. and J. J. Rowe, "Estimation of Underground Temperatures From the Silica Content of Water from Hot Springs and Wet Steam Wells," Amer. Jour. Sci. 264, 685-697 (1966).

Fournier, R. 0., D. E. White, and A. H. Truesdell, "Geochemical Indicators of Subsurface Temperature, 1, Basic Assumptions," Jour. Res. U.S. Geol. Surv. $\underline{2}, 259-262$ (1974).

Jaeger, J. C., "Application of the Theory of Heat Conduction to Geothermal Measurements," in Terrestrial Heat Flow, (American Geophysical Union, 1964), Geophysical Monograph No. 8, 7-23.

Joyner, William B., "Heat Flow in Pennsylvania and West Virginia," Geophysics 25, No. 6, 1229-1241 (1960).

Kreidler, W. Lynn, "Underground Disposal of Liquid Waste in New York," N.Y.St. Museum and Science Service, Univ. of the State of N.Y./St. Educ. Dept. Map and Chart Series No. 26, Albany, New York (1975).

Kreidler, W. Lynn, Arthur M. Van Tyne, and Kathie M. Jorgensen, "Deep Wells in New York State," N.Y.St. Museum and Science Service, Univ. of the State of N.Y./St. Educ. Dept. Bullet in 418A, Albany, New York (1972).

Middleton, M. F., "A Model for Bottom-hole Temperature Stabilization," Geophysics 44, No. 8, 1458-1462 (1974).

Revetta, F. A. and W. H. Diment, "Simple Bouguer Gravity Anomaly" Map of Western New York," N.Y.St. Museum and Science Service, Geol. Surv. Map No. 17 (1971).

Richard, L. V., "Correlation of Devonian Rocks in New York," N.Y.St. Museum and Science Service, Univ. of the State of N.Y./St. Educ. Dept., Albany, New York (1975). 
Sampson, R. J., "Surface II Graphics System," Kansas Geological Survey Computer Contribution III, Lawrence, Kansas (1975).

Swanberg, C. A. and P. Morgan, "The Linear Relation Between Temperature Based on the Silica Content of Groundwater and Regional Heat Flow: A New Heat Flow Map of the United States," PAGEOPH 117, Nos. 1,2, 227-241 (1979). 
APPENDIX A

BOTTOM-HOLE TEMPERATURE DATA FOR WELLS IN NEW YORK STATE

\author{
D.S. HODGE - STATE UNIVERSITY OF NEW YORK AT BUFFALO \\ GEOLOGICAL SCIENCES
}

\begin{tabular}{|c|c|c|c|c|c|c|c|c|c|c|c|c|c|}
\hline $\begin{array}{l}\text { JELL } \\
\text { NO }\end{array}$ & & & & $\begin{array}{l}\text { LATITUDE } \\
\text { DEGREES }\end{array}$ & $\begin{array}{l}\text { LONGITUDE } \\
\text { DEGREES }\end{array}$ & LEV & BELV & EPTH & $\begin{array}{l}\text { HT S } \\
\text { C }\end{array}$ & $\begin{array}{c}\text { STEMP } \\
\mathrm{C}\end{array}$ & $\begin{array}{c}\text { GRADIENT } \\
\mathrm{C} / \mathrm{KM}\end{array}$ & $\underset{\text { C }}{\text { HTCOR }}$ & $\begin{array}{l}\text { APGRAI } \\
\text { C/KM }\end{array}$ \\
\hline $\begin{array}{r}1 \\
2 \\
3 \\
7 \\
9 \\
15 \\
16 \\
17 \\
18 \\
19 \\
291 \\
412 \\
412 \\
1142 \\
2655 \\
3200 \\
3859 \\
3929 \\
3930 \\
3931 \\
3945 \\
3947 \\
3964 \\
3971 \\
3976 \\
3978 \\
3985 \\
3991 \\
3997 \\
4005 \\
4023 \\
4027 \\
4036 \\
4040 \\
4138 \\
4152 \\
4158 \\
4161 \\
4178 \\
4203 \\
4204 \\
4214 \\
4232 \\
1241 \\
1347 \\
4350 \\
4363 \\
1364 \\
4379 \\
4389 \\
4408 \\
4437 \\
4137 \\
4148 \\
4455\end{array}$ & $\begin{array}{r}6 \\
1 \\
11 \\
8 \\
9 \\
11 \\
5 \\
9 \\
9 \\
9 \\
6 \\
6 \\
9 \\
0 \\
10 \\
7 \\
3 \\
4 \\
3 \\
3 \\
3 \\
3 \\
4\end{array}$ & $\begin{array}{r}16 \\
13 \\
19 \\
20 \\
3 \\
9 \\
26 \\
8 \\
27 \\
19 \\
3 \\
9 \\
30 \\
0 \\
16 \\
19 \\
22 \\
11 \\
20 \\
23 \\
26 \\
9\end{array}$ & $\begin{array}{l}6 \\
7 \\
7 \\
7 \\
7 \\
7 \\
7 \\
7 \\
7 \\
7 \\
7\end{array}$ & $\begin{array}{l}41.5833 \\
41.7838 \\
41.7212 \\
41.8725 \\
40.6500 \\
42.0780 \\
42.0787 \\
42.0917 \\
12.0818 \\
42.0902 \\
42.0039 \\
42.0802 \\
42.0802 \\
42.4859 \\
42.4311 \\
42.0682 \\
42.7917 \\
42.7743 \\
42.7889 \\
42.7806 \\
42.8430 \\
42.7878 \\
42.7670 \\
42.7851 \\
42.7971 \\
42.8136 \\
42.7946 \\
42.7762 \\
42.7985 \\
42.8015 \\
42.7990 \\
42.7917 \\
42.8095 \\
42.8012 \\
42.8127 \\
42.1639 \\
42.9177 \\
42.2922 \\
42.2887 \\
42.8763 \\
42.1609 \\
41.9321 \\
42.0158 \\
42.9188 \\
42.1601 \\
42.1866 \\
42.8618 \\
42.3163 \\
42.2736 \\
42.9025 \\
42.9278 \\
42.1843 \\
42 . .1843 \\
42.9018 \\
42.3905\end{array}$ & $\begin{array}{l}76.2500 \\
76.3252 \\
76.6056 \\
76.2656 \\
76.3429 \\
77.7925 \\
77.8017 \\
77.7797 \\
77.7797 \\
77.8006 \\
77.6175 \\
77.8041 \\
77.8041 \\
79.3261 \\
79.0875 \\
79.4156 \\
77.4576 \\
77.4731 \\
77.4746 \\
77.4655 \\
77.4720 \\
77.4845 \\
77.4951 \\
77.4936 \\
77.4553 \\
77.4633 \\
77.4716 \\
77.4568 \\
77.5087 \\
77.4939 \\
77.4852 \\
77.4212 \\
77.4951 \\
77.4754 \\
77.9437 \\
79.7372 \\
76.8629 \\
79.4928 \\
79.4677 \\
76.8593 \\
79.6741 \\
74.0054 \\
79.2537 \\
76.6424 \\
79.6789 \\
76.5915 \\
77.8159 \\
75.2678 \\
74.6279 \\
76.6638 \\
76.8875 \\
79.3382 \\
79.3382 \\
76.6742 \\
75.0446\end{array}$ & $\begin{array}{l}475.8 \\
417.9 \\
339.5 \\
403.3 \\
412.4 \\
618.1 \\
624.5 \\
655.9 \\
594.7 \\
599.2 \\
627.6 \\
595.3 \\
595.3 \\
182.9 \\
417.3 \\
479.1 \\
430.4 \\
318.2 \\
363.9 \\
417.3 \\
354.2 \\
307.2\end{array}$ & $\begin{array}{l}480.4 \\
420.6 \\
341.4 \\
407.2 \\
417.3 \\
621.8 \\
628.2 \\
659.6 \\
598.3 \\
602.9 \\
631.9 \\
599.8 \\
599.8 \\
185.9 \\
421.8 \\
179.1 \\
431.0 \\
318.5 \\
364.2 \\
417.9 \\
354.5 \\
307.5 \\
324.9 \\
285.9 \\
452.3 \\
443.8 \\
423.1 \\
405.7 \\
296.9 \\
335.9 \\
351.1 \\
166.3 \\
312.4 \\
394.4 \\
280.4 \\
453.8 \\
152.4 \\
415.4 \\
417.6 \\
165.5 \\
453.2 \\
534.6 \\
481.6 \\
189.6 \\
455.4 \\
440.1 \\
188.4 \\
509.3 \\
563.0 \\
156.1 \\
153.0 \\
540.4 \\
540.4 \\
149.7 \\
456.6\end{array}$ & $\begin{array}{r}3914.5 \\
2012.9 \\
1226.2 \\
1402.1 \\
2286.6 \\
1468.2 \\
1504.2 \\
1504.5 \\
1435.9 \\
1435.3 \\
1531.9 \\
1430.7 \\
1430.7 \\
605.9 \\
937.9 \\
2042.2 \\
901.3 \\
809.2 \\
843.4 \\
891.5 \\
823.0 \\
788.2 \\
410.0 \\
765.0 \\
915.0 \\
904.3 \\
889.1 \\
886.7 \\
703.5 \\
791.0 \\
807.7 \\
937.6 \\
752.2 \\
848.6 \\
634.0 \\
1086.6 \\
492.9 \\
988.2 \\
1004.3 \\
1689.8 \\
1105.2 \\
3345.2 \\
234.1 \\
561.1 \\
1103.4 \\
1039.4 \\
489.2 \\
2355.5 \\
2753.9 \\
533.1 \\
498.3 \\
1263.4 \\
2339.6\end{array}$ & $\begin{array}{l}98.9 \\
42.8 \\
35.0 \\
40.6 \\
58.9 \\
40.6 \\
46.7 \\
38.9 \\
43.3 \\
44.4 \\
48.9 \\
43.3 \\
43.9 \\
26.1 \\
30.0 \\
48.9 \\
34.4 \\
33.3 \\
27.2 \\
31.7 \\
32.2 \\
28.3 \\
18.9 \\
29.4 \\
33.9 \\
33.9 \\
32.8 \\
32.8 \\
34.4 \\
28.9 \\
29.4 \\
35.0 \\
26.7 \\
30.0 \\
17.8 \\
42.2 \\
17.8 \\
32.8 \\
35.0 \\
48.9 \\
32.8 \\
55.0 \\
21.1 \\
30.0 \\
30.0\end{array}$ & 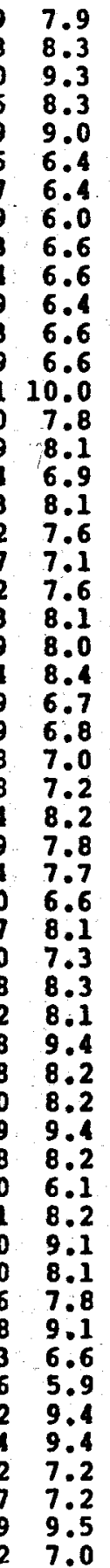 & $\begin{array}{l}23.2 \\
17.1 \\
21.0 \\
23.0 \\
21.8 \\
23.3 \\
26.8 \\
21.8 \\
25.6 \\
26.4 \\
27.7 \\
25.7 \\
26.0 \\
26.6 \\
23.7 \\
20.0 \\
30.5 \\
31.2 \\
23.3 \\
27.6 \\
29.9 \\
25.6 \\
26.5 \\
27.6 \\
29.7 \\
30.0 \\
29.0 \\
28.8 \\
37.3 \\
26.6 \\
26.9 \\
30.3 \\
24.7 \\
26.8 \\
14.9 \\
31.4 \\
17.0 \\
24.9 \\
26.7 \\
23.4 \\
22.3\end{array}$ & $\begin{array}{r}26.3 \\
13.0 \\
7.5 \\
8.7 \\
14.9 \\
9.2 \\
9.4 \\
9.4 \\
9.0 \\
9.0 \\
9.6 \\
8.9 \\
8.9 \\
3.2 \\
5.5 \\
13.2 \\
5.2 \\
4.6 \\
4.8 \\
5.2 \\
4.7 \\
4.4 \\
1.8 \\
4.3 \\
5.3 \\
5.3 \\
5.1 \\
5.1 \\
3.9 \\
4.5 \\
4.6 \\
5.5\end{array}$ & $\begin{array}{l}0.0 \\
3.6 \\
7.1 \\
9.2 \\
8.3 \\
9.5 \\
3.1 \\
8.1 \\
1.8 \\
2.6 \\
4.0 \\
1.9 \\
2.3 \\
1.9 \\
9.5 \\
6.4 \\
6.3 \\
6.9 \\
9.0 \\
3.4 \\
5.6 \\
1.2 \\
0.9 \\
3.1 \\
5.5 \\
5.8\end{array}$ \\
\hline
\end{tabular}


APPENDIX A (Cont)

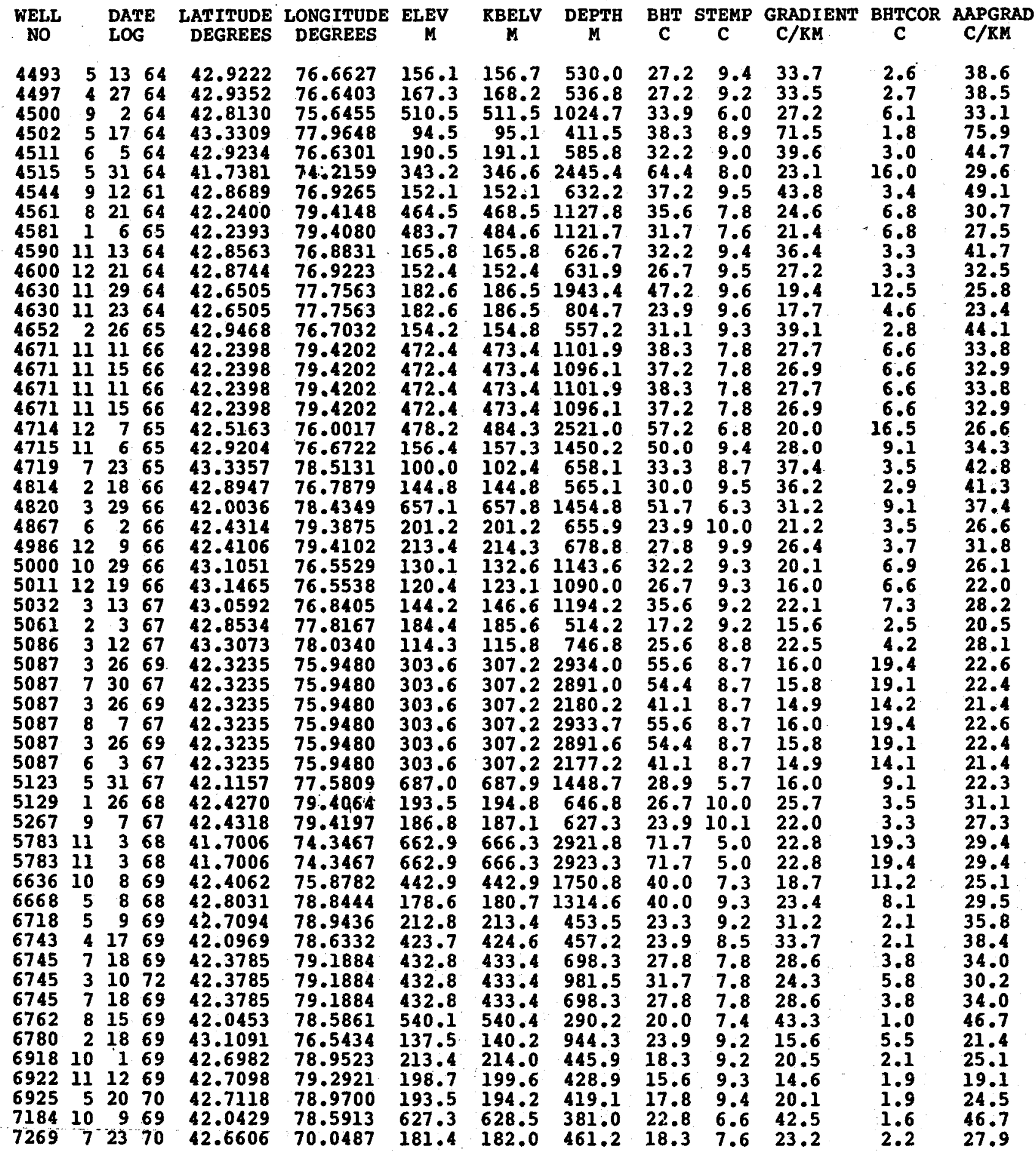


APPENDIX A (Cont)

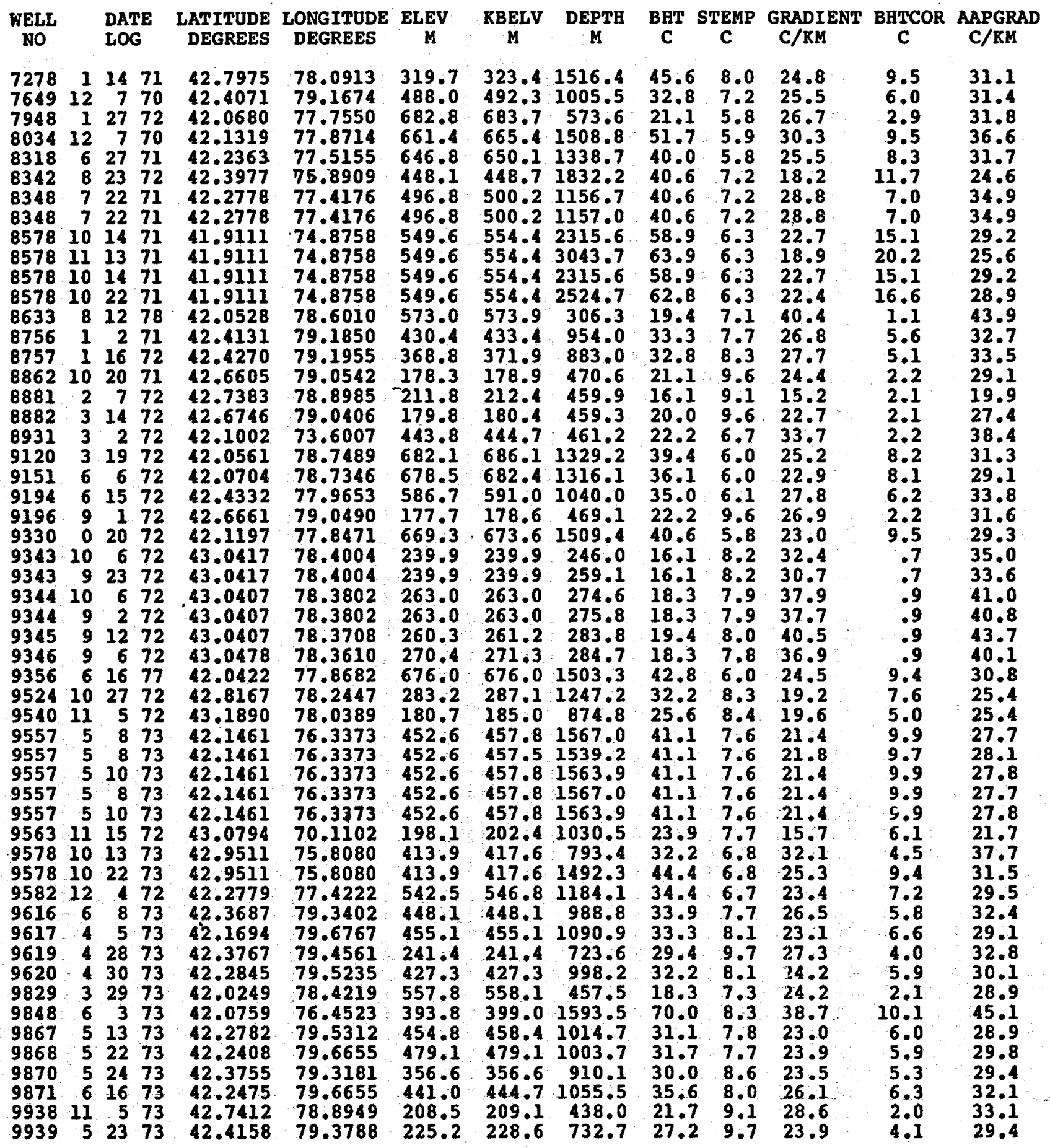


APPENDIX A (Cont)

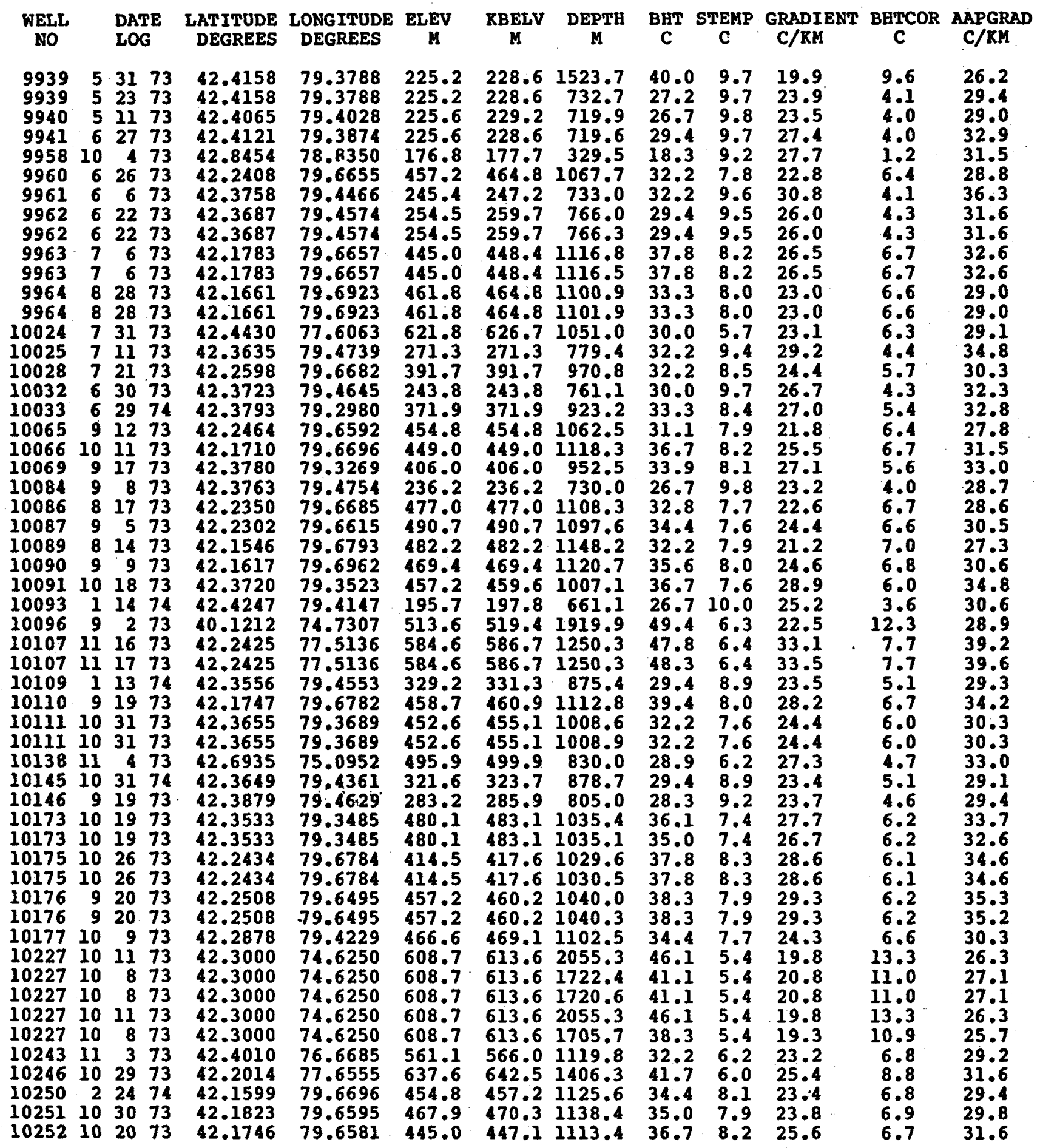


APPENDIX A (Cont)

\begin{tabular}{|c|c|c|c|c|c|c|c|c|c|c|c|c|}
\hline $\begin{array}{l}\text { WELL } \\
\text { NO }\end{array}$ & & ITE & $\begin{array}{l}\text { ATITUDE } \\
\text { DEGREES }\end{array}$ & $\begin{array}{l}\text { LONG ITUDE } \\
\text { DEGREES }\end{array}$ & $\underset{M}{\text { ELEV }}$ & $\underset{M}{\operatorname{BELV}}$ & $\underset{M}{E P T H}$ & $\underset{\mathrm{C}}{\mathrm{BHT}}$ & $\underset{\mathrm{C}}{\operatorname{STEMP}}$ & $\begin{array}{l}\text { GRADIENT } \\
\mathrm{C} / \mathrm{KM}\end{array}$ & $\underset{\mathrm{C}}{\mathrm{BHTCOR}}$ & $\begin{array}{l}\text { AAPGRAD } \\
\text { C/RM }\end{array}$ \\
\hline $\begin{array}{l}10253 \\
10254 \\
10258 \\
10259 \\
10263 \\
10263 \\
10278 \\
10278 \\
10285 \\
10285 \\
10286 \\
10286 \\
10287 \\
10287 \\
10288 \\
10289 \\
10290 \\
10292 \\
10293 \\
10293 \\
10298 \\
10298 \\
10299 \\
10316 \\
10316 \\
10332 \\
10332 \\
10335 \\
10335 \\
10345 \\
10370 \\
10375 \\
10382 \\
10389 \\
10406 \\
10407 \\
10417 \\
10448 \\
10455 \\
10456 \\
10457 \\
10458 \\
10460 \\
10461 \\
10469 \\
10470 \\
10477 \\
10479 \\
10484 \\
10485 \\
10485 \\
10486 \\
10486 \\
10489 \\
10528 \\
\end{array}$ & $\begin{array}{l}1 \\
1 \\
1 \\
1 \\
1\end{array}$ & 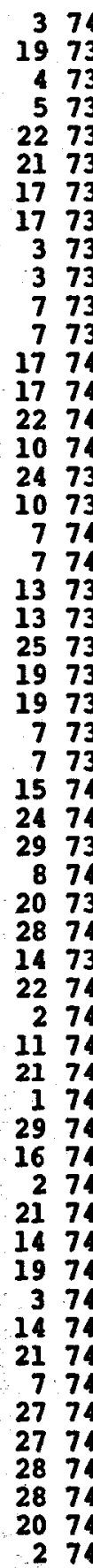 & $\begin{array}{l}42.3547 \\
42.3499 \\
42.2404 \\
42.2442 \\
42.4424 \\
42.4424 \\
42.1727 \\
42.1727 \\
42.3690 \\
42.3690 \\
42.3566 \\
42.3566 \\
42.3504 \\
42.3504 \\
42.3518 \\
42.3667 \\
42.3622 \\
42.2457 \\
42.2480 \\
42.2480 \\
42.3502 \\
42.3502 \\
42.3605 \\
42.2289 \\
42.2289 \\
42.1781 \\
42.1781 \\
42.1690 \\
42.1690 \\
42.2455 \\
42.1847 \\
42.4235 \\
42.1595 \\
42.1546 \\
42.2488 \\
42.2333 \\
42.4233 \\
42.4542 \\
42.4134 \\
42.3624 \\
42.2284 \\
42.2371 \\
42.36244 \\
42.3572 \\
42.2721 \\
42.2648 \\
42.4115 \\
42.3460 \\
42.3473 \\
42.2234 \\
42.2234 \\
42.2295 \\
42.2295 \\
42.3437 \\
42.4075\end{array}$ & $\begin{array}{l}79.3638 \\
79.3559 \\
79.6940 \\
77.2167 \\
74.6206 \\
74.6206 \\
79.6990 \\
79.6990 \\
79.4795 \\
79.4795 \\
79.4854 \\
79.4854 \\
79.4889 \\
79.4889 \\
79.4652 \\
79.4845 \\
79.4805 \\
79.6718 \\
79.6780 \\
79.6780 \\
79.3689 \\
79.3689 \\
79.3328 \\
79.6731 \\
79.6731 \\
79.7045 \\
79.7045 \\
76.6745 \\
76.6745 \\
79.6920 \\
79.7063 \\
79.3762 \\
79.6577 \\
79.6793 \\
79.6979 \\
79.6857 \\
79.3155 \\
79.3536 \\
79.3411 \\
79.3756 \\
79.6865 \\
79.6990 \\
79.3756 \\
79.3804 \\
79.5279 \\
79.5166 \\
79.4432 \\
79.3631 \\
79.4835 \\
79.6584 \\
79.6584 \\
79.6493 \\
79.6493\end{array}$ & $\begin{array}{l}358.4 \\
475.5 \\
390.1 \\
323.1 \\
638.6 \\
638.6 \\
462.1 \\
462.1 \\
241.7 \\
241.7 \\
262.7 \\
262.7 \\
281.3 \\
281.3 \\
329.8 \\
232.3 \\
249.3 \\
427.9 \\
399.6 \\
399.6 \\
469.7 \\
469.7 \\
411.5 \\
472.4 \\
472.4 \\
463.9 \\
463.9 \\
432.2 \\
432.2 \\
370.3 \\
447.4 \\
226.2 \\
447.4 \\
482.2 \\
357.8 \\
424.3 \\
266.7 \\
199.3 \\
287.1 \\
466.3 \\
443.2 \\
389.2 \\
466.3 \\
473.4 \\
461.8 \\
485.9 \\
199.6 \\
455.1 \\
318.8 \\
496.8 \\
496.8 \\
509.0 \\
509.0 \\
313.3 \\
204.2\end{array}$ & $\begin{array}{l}360.6 \\
477.9 \\
391.7 \\
325.8 \\
642.5 \\
642.5 \\
463.6 \\
463.6 \\
243.8 \\
243.8 \\
264.9 \\
264.9 \\
287.1 \\
287.1 \\
337.4 \\
234.7 \\
251.5 \\
427.9 \\
402.6 \\
402.6 \\
472\end{array}$ & $\begin{array}{r}905.0 \\
1030.2 \\
984.2 \\
887.3 \\
1304.5 \\
1303.6 \\
1126.2 \\
1128.1 \\
766.9 \\
767.2 \\
781.8 \\
782.1 \\
814.4 \\
815.6 \\
852.8 \\
762.0 \\
766.9 \\
1040.3 \\
1003.4 \\
1002.2 \\
1023.2 \\
1022.6 \\
961.9 \\
1080.8 \\
1080.2 \\
1128.7 \\
1128.4 \\
1678.8 \\
3234.2 \\
985.7 \\
1085.1 \\
686.7 \\
1127.8 \\
1147.6 \\
956.5 \\
1030.2 \\
728.2 \\
698.6 \\
770.2 \\
1019.6 \\
1059.5 \\
976.9 \\
1016.8 \\
1038.1 \\
1036.9 \\
1060.4 \\
674.8 \\
1016.2 \\
852.5 \\
1114.7 \\
1115.9 \\
1131.7 \\
1131.7\end{array}$ & $\begin{array}{l}32.2 \\
36.7 \\
36.7 \\
28.9 \\
37.2 \\
37.2 \\
38.9 \\
38.9 \\
31.7 \\
31.7 \\
28.9 \\
28.9 \\
27.8 \\
27.8 \\
26.7 \\
29.4 \\
31.7 \\
32.2 \\
26.7\end{array}$ & $\begin{array}{l}8.6 \\
7.44 \\
8.6 \\
8.9 \\
5.0 \\
5.0 \\
8.0 \\
8.00 \\
9.77 \\
9.77 \\
9.5 \\
9.5 \\
9.3 \\
9.3 \\
8.8 \\
9.8 \\
9.6 \\
8.2 \\
8.4 \\
8.44 \\
7.5 \\
7.5 \\
8.0 \\
7.8 \\
7.8 \\
8.0 \\
8.0\end{array}$ & $\begin{array}{l}26.1 \\
28.4 \\
28.6 \\
22.5 \\
24.7 \\
24.7 \\
27.4 \\
27.4 \\
28.6 \\
28.6 \\
24.8 \\
24.8 \\
22.7 \\
22.6 \\
20.9 \\
25.8 \\
28.7 \\
23.1 \\
18.2 \\
18.2 \\
28.5\end{array}$ & 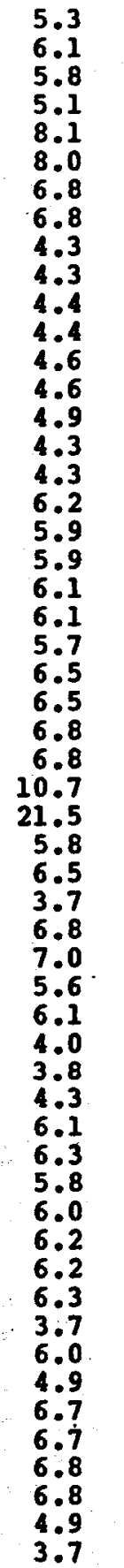 & $\begin{array}{l}28.2 \\
28.1 \\
29.4 \\
31.9 \\
26.3 \\
33.9\end{array}$ \\
\hline
\end{tabular}


APPENDIX A (Cont)

\begin{tabular}{|c|c|c|c|c|c|c|c|c|c|c|c|c|}
\hline $\begin{array}{l}\text { WELL } \\
\text { NO }\end{array}$ & & $\begin{array}{l}\text { DATE } \\
\text { LOG }\end{array}$ & $\begin{array}{l}\text { LATITUDE } \\
\text { DEGREES }\end{array}$ & $\begin{array}{l}\text { LONGITUDE } \\
\text { DEGREES }\end{array}$ & $\underset{\mathbf{M}}{\text { ELEV }}$ & $\underset{M}{\text { RBELV }}$ & $\underset{M}{\text { DEPTH }}$ & $\underset{\mathrm{C}}{\mathrm{BHT}} \mathrm{s}$ & $\underset{C}{\text { STEMP }}$ & $\begin{array}{l}\text { GRADIENT } \\
\mathrm{C} / \mathrm{KM}\end{array}$ & $\underset{\mathrm{C}}{\mathrm{HTCOR}}$ & $\begin{array}{l}\text { AAPGRAD } \\
\text { C/KM }\end{array}$ \\
\hline $\begin{array}{l}10530 \\
10531 \\
10532 \\
10532 \\
10534 \\
10535 \\
10550 \\
10550 \\
10554 \\
10564 \\
10564 \\
10566 \\
10566 \\
10577 \\
10578 \\
10578 \\
10579 \\
10580 \\
10580 \\
10584 \\
10584 \\
10585 \\
10596 \\
10597 \\
10601 \\
10605 \\
10607 \\
10608 \\
10608 \\
10608 \\
10655 \\
10656 \\
10656 \\
10658 \\
10700 \\
10701 \\
10702 \\
10705 \\
10706 \\
10725 \\
10756 \\
10756 \\
10757 \\
10771 \\
10836 \\
10836 \\
10838 \\
10855 \\
10872 \\
10873 \\
10875 \\
10877 \\
10878 \\
10883 \\
10884\end{array}$ & $\begin{array}{r}3 \\
3 \\
4 \\
3 \\
3 \\
3 \\
3 \\
33 \\
3 \\
3 \\
3 \\
4 \\
3 \\
3 \\
5 \\
3 \\
3 \\
4 \\
4 \\
11 \\
4 \\
4\end{array}$ & $\begin{array}{rl}6 & 74 \\
12 & 74 \\
12 & 74 \\
12 & 74 \\
2 & 74 \\
12 & 74 \\
25 & 74 \\
25 & 74 \\
13 & 74 \\
19 & 74 \\
19 & 74 \\
25 & 74 \\
25 & 74 \\
10 & 74 \\
26 & 74 \\
26 & 74 \\
26 & 74 \\
23 & 74 \\
23 & 74 \\
1 & 74 \\
1 & 74 \\
15 & 74 \\
1 & 74 \\
7 & 74 \\
13 & 74 \\
23 & 74 \\
28 & 74 \\
3 & 74 \\
3 & 74 \\
3 & 74 \\
20 & 74 \\
24 & 74 \\
24 & 74 \\
8 & 74 \\
21 & 74 \\
16 & 74 \\
9 & 74 \\
30 & 74 \\
19 & 74 \\
19 & 74 \\
6 & 74 \\
6 & 74 \\
7 & 74 \\
1 & 74 \\
10 & 74 \\
11 & 74 \\
24 & 74 \\
20 & 77 \\
27 & 74 \\
28 & 74 \\
10 & 74 \\
17 & 74 \\
27 & 74 \\
6 & 74 \\
26 & 74\end{array}$ & $\begin{array}{l}42.3616 \\
42.3451 \\
42.2250 \\
42.2250 \\
42.4859 \\
42.3818 \\
42.2286 \\
42.2286 \\
42.3363 \\
42.3895 \\
42.3895 \\
42.2876 \\
42.2876 \\
42.4113 \\
42.4018 \\
42.4018 \\
42.3470 \\
42.3324 \\
42.3324 \\
42.3667 \\
42.3667 \\
42.3586 \\
42.3379 \\
42.2159 \\
42.3876 \\
42.3412 \\
42.4511 \\
42.3172 \\
42.3172 \\
42.3172 \\
42.2104 \\
42.3892 \\
42.3892 \\
42.3832 \\
42.3552 \\
42.8763 \\
42.8766 \\
42.1899 \\
42.1932 \\
42.5298 \\
42.3644 \\
42.3644 \\
42.2705 \\
42.1655 \\
42.6650 \\
42.6650 \\
42.3290 \\
42.0210 \\
42.0539 \\
42.0559 \\
42.1503 \\
42.2953 \\
42.3357 \\
42.3665 \\
42.3697\end{array}$ & $\begin{array}{l}79.4417 \\
79.3739 \\
79.6645 \\
79.6645 \\
79.3261 \\
79.4741 \\
79.6517 \\
79.6517 \\
79.3733 \\
79.4752 \\
79.4752 \\
79.5339 \\
79.5339 \\
78.8739 \\
79.4451 \\
79.4451 \\
79.4746 \\
79.5154 \\
79.5154 \\
79.4924 \\
79.4924 \\
79.4326 \\
79.3801 \\
79.6578 \\
79.4386 \\
79.4439 \\
75.4848 \\
75.6714 \\
75.6714 \\
75.6714 \\
79.6575 \\
79.4500 \\
79.4500 \\
79.4432 \\
79.4953 \\
76.7064 \\
76.6932 \\
79.6605 \\
79.6551 \\
75.2468 \\
79.4480 \\
79.4480 \\
79.5481 \\
79.6660 \\
78.5612 \\
78.5612 \\
75.3750 \\
78.6008 \\
79.4637 \\
79.0773 \\
79.6861 \\
79.4667 \\
79.4532 \\
79.2977 \\
79.4422\end{array}$ & $\begin{array}{l}341.1 \\
410.6 \\
490.1 \\
490.1 \\
182.9 \\
222.5 \\
496.2 \\
496.2 \\
403.3 \\
201.2 \\
201.2 \\
431.0 \\
431.0 \\
445.0 \\
204.5 \\
204.5 \\
340.5 \\
402.3 \\
402.3 \\
235.0 \\
235.0 \\
338.3 \\
405.7 \\
501.1 \\
235.0 \\
418.8 \\
523.3 \\
426.7 \\
426.7 \\
426.7 \\
501.4 \\
217.9 \\
217.9 \\
241.1 \\
249.0 \\
125.3 \\
138.4 \\
472.4 \\
474.9 \\
347.2 \\
318.5 \\
318.5 \\
420.6 \\
437.4 \\
321.6 \\
321.6 \\
365.2 \\
558.4 \\
473.0 \\
440.1 \\
486.8 \\
443.8 \\
463.0 \\
420.6 \\
276.1\end{array}$ & $\begin{array}{l}343.2 \\
413.0 \\
492.9 \\
492.9 \\
185.9 \\
225.9 \\
496.2 \\
496.2 \\
405.4 \\
204.5 \\
204.5 \\
434.0 \\
434.0 \\
448.1 \\
204.5 \\
204.5 \\
342.3 \\
402.3 \\
402.3 \\
237.1 \\
237.1 \\
340.5 \\
405.7 \\
504.1 \\
235.0 \\
420.9 \\
528.8 \\
431.3 \\
431.3 \\
431.3 \\
504.4 \\
221.3 \\
221.3 \\
241.1 \\
251.2 \\
128.3 \\
141.4 \\
475.5 \\
477.9 \\
352.3 \\
321.0 \\
321.0 \\
423.1 \\
440.4 \\
322.5 \\
322.5 \\
370.3 \\
559.9 \\
476.4 \\
443.8 \\
489.8 \\
445.9 \\
463.0 \\
423.1 \\
279.0\end{array}$ & $\begin{array}{r}883.9 \\
973.8 \\
1108.3 \\
1107.6 \\
708.4 \\
714.1 \\
1116.5 \\
1117.1 \\
962.6 \\
697.7 \\
696.5 \\
1012.2 \\
1012.5 \\
1045.8 \\
686.1 \\
685.8 \\
878.7 \\
969.0 \\
968.3 \\
753.5 \\
752.6 \\
882.4 \\
964.7 \\
1150.3 \\
801.6 \\
981.5 \\
1611.2 \\
2008.6 \\
2010.8 \\
2015.0 \\
1200.9 \\
712.0 \\
711.4 \\
730.0 \\
770.2 \\
550.8 \\
553.5 \\
1108.6 \\
1119.2 \\
1212.5 \\
858.0 \\
860.1 \\
1028.7 \\
1116.8 \\
666.9 \\
354.5 \\
1778.5 \\
1272.2 \\
1312.5 \\
1398.7 \\
1153.7 \\
1004.3 \\
1034.5 \\
993.0 \\
801.3\end{array}$ & $\begin{array}{l}30.0 \\
29.4 \\
35.6 \\
35.6 \\
28.9 \\
29.4 \\
.30 .0 \\
30.0 \\
28.3 \\
26.7 \\
26.7 \\
28.9 \\
28.9 \\
25.6 \\
25.6 \\
25.6 \\
32.8 \\
26.7 \\
26.7 \\
25.6 \\
25.6 \\
30.0 \\
29.4 \\
36.1 \\
29.4 \\
30.0 \\
32.8 \\
43.3 \\
43.3 \\
43.3 \\
37.8 \\
27.8 \\
27.8 \\
28.3 \\
28.9 \\
23.9 \\
27.8 \\
35.6 \\
33.9 \\
36.7 \\
31.1 \\
31.1 \\
35.6 \\
36.1 \\
31.1 \\
20.6 \\
39.4 \\
28.3 \\
38.9 \\
37.8 \\
36.7 \\
33.3 \\
34.4 \\
37.2 \\
28.9\end{array}$ & $\begin{array}{r}8.7 \\
8.1 \\
7.6 \\
7.6 \\
10.0 \\
9.8 \\
7.6 \\
7.6 \\
8.2 \\
10.0 \\
10.0 \\
8.0 \\
8.0 \\
7.6 \\
10.0 \\
10.0 \\
8.8 \\
8.2 \\
8.2 \\
9.8 \\
9.8 \\
8.8 \\
8.2 \\
7.5 \\
9.7 \\
8.0 \\
6.3 \\
7.4 \\
7.4 \\
7.4 \\
7.5 \\
9.9 \\
9.9 \\
9.7 \\
9.7 \\
9.7 \\
9.6 \\
7.9 \\
7.8 \\
7.9 \\
9.0 \\
9.0 \\
8.2 \\
8.3 \\
8.2 \\
8.2 \\
8.0 \\
7.3 \\
8.2 \\
8.4 \\
7.8 \\
7.9 \\
7.6 \\
7.9 \\
9.4\end{array}$ & $\begin{array}{l}24.0 \\
21.9 \\
25.2 \\
25.2 \\
26.7 \\
27.4 \\
20.1 \\
20.1 \\
20.9 \\
23.8 \\
23.9 \\
20.6 \\
20.6 \\
17.2 \\
22.7 \\
22.7 \\
27.3 \\
19.0 \\
19.0 \\
21.0 \\
21.0 \\
24.1 \\
22.0 \\
24.9 \\
24.6 \\
22.4 \\
16.4 \\
17.9 \\
17.8 \\
17.8 \\
25.2 \\
25.2 \\
25.2 \\
25.5 \\
25.0 \\
25.7 \\
32.8 \\
25.0 \\
23.3 \\
23.7 \\
25.8 \\
25.8 \\
26.6 \\
24.9 \\
34.3 \\
34.7 \\
17.7 \\
16.5 \\
23.4 \\
21.0 \\
25.0 \\
25.3 \\
25.3 \\
29.5 \\
24.4\end{array}$ & $\begin{array}{r}5.1 \\
5.7 \\
6.7 \\
6.7 \\
3.9 \\
3.9 \\
6.7 \\
6.7 \\
5.7 \\
3.8 \\
3.8 \\
6.0 \\
6.0 \\
6.2 \\
3.7 \\
3.7 \\
5.1 \\
5.7 \\
5.7 \\
4.2 \\
4.2 \\
5.1 \\
5.7 \\
7.0 \\
4.5 \\
5.8 \\
10.2 \\
13.0 \\
13.0 \\
13.0 \\
7.3 \\
3.9 \\
3.9 \\
4.0 \\
4.3 \\
2.8 \\
2.8 \\
6.7 \\
6.8 \\
7.4 \\
4.9 \\
4.9 \\
6.1 \\
6.7 \\
3.6 \\
1.4 \\
11.4 \\
7.8 \\
8.1 \\
8.7 \\
7.0 \\
6.0 \\
6.2 \\
5.9 \\
4.5\end{array}$ & $\begin{array}{r}29.8 \\
27.8 \\
31.2 \\
31.3 \\
32.3 \\
32.9 \\
26.1 \\
26.1 \\
26.8 \\
29.3 \\
29.3 \\
26.5 \\
26.5 \\
23.1 \\
28.1 \\
28.1 \\
33.1 \\
24.9 \\
24.9 \\
26.5 \\
26.5 \\
29.8 \\
27.9 \\
30.9 \\
30.2 \\
28.3 \\
22.8 \\
24.3 \\
24.3 \\
24.3 \\
31.3 \\
30.6 \\
30.7 \\
31.1 \\
30.6 \\
30.7 \\
37.9 \\
31.0 \\
29.3 \\
29.8 \\
31.6 \\
31.5 \\
32.6 \\
31.0 \\
39.7 \\
38.7 \\
24.1 \\
22.7 \\
29.6 \\
27.2 \\
31.1 \\
31.3 \\
31.9 \\
35.4 \\
30.0\end{array}$ \\
\hline
\end{tabular}


APPENDIX A (Cont)

\begin{tabular}{|c|c|c|c|c|c|c|c|c|c|c|c|c|c|}
\hline $\begin{array}{l}\text { WELL } \\
\text { NO }\end{array}$ & & & & $\begin{array}{l}\text { LATITUDE } \\
\text { DEGREES }\end{array}$ & $\begin{array}{l}\text { LONGITUDE } \\
\text { DEGREES }\end{array}$ & $\underset{M}{\text { ELEV }}$ & $\underset{\mathbf{M}}{\text { RBELV }}$ & EPTH & $\underset{\mathrm{C}}{\mathrm{BHT}} \mathrm{s}$ & $\underset{C}{\text { STEMP }}$ & $\begin{array}{l}\text { GRADIENI } \\
\text { C/KM }\end{array}$ & $\begin{array}{l}\text { 3HTCOR } \\
\text { C }\end{array}$ & $\begin{array}{l}\text { AAPGRAD } \\
\text { C/KM }\end{array}$ \\
\hline $\begin{array}{l}10895 \\
10895 \\
10896 \\
10898 \\
10899 \\
10899 \\
10900 \\
10917 \\
10923 \\
10931 \\
10937 \\
10937 \\
10939 \\
10943 \\
10944 \\
10944 \\
10945 \\
10946 \\
10948 \\
10948 \\
10949 \\
10949 \\
10955 \\
10957 \\
10960 \\
10962 \\
10967 \\
10968 \\
10972 \\
10973 \\
10973 \\
10993 \\
10999 \\
11000 \\
11000 \\
11002 \\
11002 \\
11004 \\
11005 \\
11021 \\
11022 \\
11022 \\
11023 \\
11023 \\
11027 \\
11030 \\
11031 \\
11032 \\
11033 \\
11036 \\
11038 \\
11040 \\
11041 \\
\end{array}$ & $\begin{array}{r}9 \\
6 \\
6 \\
6 \\
6 \\
8 \\
6 \\
6 \\
7 \\
7 \\
7 \\
7 \\
7 \\
7 \\
7 \\
7 \\
7 \\
7 \\
10 \\
11\end{array}$ & $\begin{array}{r}26 \\
27 \\
13 \\
13 \\
19 \\
11 \\
22 \\
22 \\
5 \\
3 \\
7 \\
24 \\
5 \\
5 \\
25 \\
8 \\
15 \\
15 \\
22 \\
19 \\
18 \\
18 \\
16\end{array}$ & & $\begin{array}{l}42.3697 \\
42.3366 \\
42.3580 \\
42.3580 \\
42.1530 \\
42.1707 \\
42.2178 \\
42.2178 \\
42.1516 \\
42.7612 \\
42.3439 \\
42.2008 \\
42.3447 \\
42.3447 \\
42.7668 \\
42.1685 \\
42.3345 \\
42.3345 \\
42.3525 \\
42.4278 \\
42.1525 \\
42.1525 \\
42.2244 \\
42.2244 \\
42.2232 \\
42.3291 \\
42.8566 \\
42.4160 \\
42.3486 \\
42.3678 \\
42.3557 \\
42.1773 \\
42.1773 \\
42.3507 \\
42.1880 \\
42.1919 \\
42.1919 \\
42.5575 \\
42.5575 \\
42.3422 \\
42.3368 \\
42.3366 \\
42.3176 \\
42.3176 \\
42.1486 \\
42.1486 \\
42.4634 \\
42.1776 \\
42.3734 \\
42.4097 \\
42.3340 \\
42.4128 \\
42.1024 \\
42.1921 \\
42.1941\end{array}$ & $\begin{array}{l}79.2992 \\
79.2992 \\
79.6921 \\
79.6458 \\
79.6819 \\
79.6819 \\
79.6683 \\
78.4442 \\
79.4621 \\
79.56655 \\
79.4012 \\
79.4012 \\
78.4179 \\
79.6892 \\
79.3983 \\
79.3983 \\
79.3317 \\
79.3019 \\
79.6573 \\
79.6573 \\
79.6956 \\
79.6956 \\
79.6839 \\
79.3926 \\
78.7851 \\
78.8353 \\
79.4453 \\
79.6916 \\
79.2508 \\
79.7103 \\
79.7103 \\
79.3780 \\
79.6924 \\
79.68663 \\
79.66863 \\
78.7447 \\
78.7447 \\
79.4083 \\
79.4052 \\
79.4454 \\
79.3813 \\
79.3813 \\
79.7102 \\
79.7102 \\
77.3177 \\
79.66920 \\
79 . .4940 \\
79 . .2619 \\
79.48833 \\
79.0729 \\
79.14462 \\
79.6693 \\
79.6798\end{array}$ & $\begin{array}{l}276.1 \\
404.8 \\
415.1 \\
415.1 \\
488.6 \\
463.9 \\
475.2 \\
475.2 \\
479.8 \\
477.0 \\
442.9 \\
488.6 \\
405.4 \\
405.4 \\
457.2 \\
455.4 \\
423.1 \\
423.1 \\
407.8 \\
265.2 \\
454.8 \\
454.8 \\
417.0 \\
417.0 \\
459.3 \\
415.4 \\
159.1 \\
426.7 \\
414.5 \\
422.8 \\
424.9 \\
439.2 \\
439.2 \\
420.6 \\
475.5 \\
487.7 \\
487.7 \\
509.0 \\
509.0 \\
454.8 \\
414.8 \\
453.5 \\
402.3 \\
402.3 \\
460.6 \\
460.6 \\
443.5 \\
468.8 \\
218.5 \\
451.1 \\
424.6 \\
395.0 \\
384.4 \\
1079.0 \\
435.9\end{array}$ & $\begin{array}{l}279.2 \\
406.9 \\
417.6 \\
417.6 \\
491.6 \\
468.5 \\
475.2 \\
475.2 \\
482.8 \\
480.7 \\
445.0 \\
491.9 \\
407.8 \\
407.8 \\
460.6 \\
458.4 \\
452.9 \\
452.9 \\
410.3 \\
268.2 \\
457.8 \\
457.8 \\
420.0 \\
420.0 \\
462.4 \\
418.8 \\
160.0 \\
429.8 \\
416.7 \\
425.2 \\
427.0 \\
442.3 \\
442.3 \\
420.9 \\
478.5 \\
490.7 \\
490.7 \\
512.7 \\
512.7 \\
458.1 \\
417.9 \\
455.7 \\
404.5 \\
404.5 \\
463.6 \\
463.6 \\
447.1 \\
471.8 \\
220.7 \\
453.5 \\
426.7 \\
397.5 \\
387.7 \\
1082.0 \\
438.9\end{array}$ & $\begin{array}{r}808.6 \\
963.8 \\
996.4 \\
995.8 \\
1151.8 \\
1137.2 \\
1112.5 \\
1112.8 \\
1166.5 \\
681.5 \\
985.1 \\
1143.0 \\
980.8 \\
980.5 \\
1652.0 \\
1083.3 \\
979.6\end{array}$ & $\begin{array}{l}30.0 \\
30.0 \\
36.7 \\
38.3 \\
35.6 \\
35.6 \\
36.7 \\
35.0 \\
38.9 \\
35.0 \\
33.3 \\
33.3 \\
42.2 \\
37.2 \\
33.3 \\
33.3 \\
30.0\end{array}$ & $\begin{array}{l}9.4 \\
8.2 \\
8.0 \\
8.0 \\
7.8 \\
8.0 \\
7.8 \\
7.8 \\
7.9 \\
6.5 \\
7.8 \\
7.7 \\
8.1 \\
8.1 \\
6.7 \\
8.1 \\
7.7 \\
7.7 \\
8.1 \\
9.3 \\
8.1\end{array}$ & $\begin{array}{l}24.2 \\
25.5 \\
22.1 \\
22.1 \\
25.1 \\
26.7 \\
24.9 \\
24.9 \\
24.7 \\
41.9 \\
31.6 \\
23.9 \\
25.7 \\
25.7 \\
21.5 \\
26.9 \\
26.1 \\
26.1 \\
22.6 \\
18.8 \\
24.0 \\
24.0 \\
25.1 \\
25.1 \\
25.4 \\
31.0 \\
28.4 \\
24.6\end{array}$ & $\begin{array}{r}5 \\
5 \\
7 \\
6 \\
6 \\
6 \\
7 \\
3 \\
5 \\
6 \\
5 \\
5 \\
10 \\
6 \\
5 \\
5 \\
5 \\
4 \\
7\end{array}$ & $\begin{array}{l}29.8 \\
31.4 \\
28.0 \\
28.0 \\
31.1 \\
32.7 \\
31.0 \\
31.0 \\
30.7 \\
47.3 \\
37.5 \\
30.0 \\
31.6 \\
31.6 \\
27.9 \\
32.9\end{array}$ \\
\hline
\end{tabular}


APPENDIX A (Cont)

\begin{tabular}{|c|c|c|c|c|c|c|c|c|c|c|c|c|}
\hline $\begin{array}{l}\text { WELL } \\
\text { NO }\end{array}$ & & $\begin{array}{l}\text { DATE } \\
\text { LOG }\end{array}$ & $\begin{array}{c}\text { LATITUDE } \\
\text { DEGREES }\end{array}$ & $\begin{array}{c}\text { LONG ITUDE } \\
\text { DEGREES }\end{array}$ & $\underset{M}{\text { ELEV }}$ & $\underset{M}{\text { KBELV }}$ & $\underset{M}{\text { DEPTH }}$ & $\underset{\mathrm{C}}{\mathrm{BHT}} \mathrm{S}$ & $\begin{array}{l}\text { PEMP } \\
\text { C }\end{array}$ & $\begin{array}{l}\text { GRADIENI } \\
C / R M\end{array}$ & $\underset{\mathrm{C}}{\mathrm{BHTOR}}$ & $\begin{array}{l}\text { AAPGRAD } \\
\text { C/RM }\end{array}$ \\
\hline $\begin{array}{l}11042 \\
11043 \\
11054 \\
11059 \\
11063 \\
11066 \\
11067 \\
11067 \\
11069 \\
11069 \\
11076 \\
11079 \\
11094 \\
11096 \\
11097 \\
11098 \\
11105 \\
11110 \\
11111 \\
11112 \\
11114 \\
11117 \\
11118 \\
11119 \\
11120 \\
11121 \\
11126 \\
11130 \\
11131 \\
11132 \\
11133 \\
11135 \\
11136 \\
11137 \\
11138 \\
11146 \\
11147 \\
11148 \\
11152 \\
11153 \\
11154 \\
11162 \\
11163 \\
11165 \\
11167 \\
11169 \\
11170 \\
11178 \\
11179 \\
11180 \\
11181 \\
11182 \\
11183 \\
11192 \\
11193\end{array}$ & $\begin{array}{r}9 \\
9 \\
9 \\
10 \\
11 \\
10 \\
10 \\
10 \\
10 \\
10 \\
11 \\
9 \\
9 \\
10 \\
10\end{array}$ & $\begin{array}{rl}8 & 74 \\
2 & 74 \\
12 & 74 \\
4 & 74 \\
14 & 74 \\
23 & 74 \\
8 & 74 \\
8 & 74 \\
29 & 74 \\
29 & 74 \\
3 & 74 \\
16 & 74 \\
26 & 73 \\
27 & 74 \\
9 & 74 \\
1 & 74 \\
12 & 74 \\
11 & 74 \\
3 & 74 \\
29 & 74 \\
11 & 75 \\
8 & 74 \\
31 & 75 \\
8 & 75 \\
17 & 74 \\
2 & 74 \\
18 & 74 \\
6 & 74 \\
19 & 74 \\
1 & 74 \\
11 & 74 \\
7 & 74 \\
3 & 74 \\
9 & 74 \\
30 & 74 \\
8 & 74 \\
31 & 74 \\
16 & 74 \\
25 & 74 \\
9 & 74 \\
24 & 74 \\
22 & 74 \\
11 & 74 \\
21 & 74 \\
13 & 74 \\
19 & 74 \\
12 & 74 \\
27 & 74 \\
4 & 74 \\
16 & 75 \\
6 & 75 \\
7 & 75 \\
9 & 75 \\
8 & 75 \\
19 & 74\end{array}$ & $\begin{array}{l}42.1588 \\
42.1874 \\
42.3610 \\
42.3185 \\
42.2482 \\
42.4032 \\
42.4283 \\
42.4283 \\
42.3892 \\
42.3892 \\
42.4161 \\
42.1466 \\
42.3223 \\
42.1909 \\
42.3255 \\
42.3284 \\
42.2202 \\
42.1565 \\
42.3351 \\
42.3430 \\
42.5976 \\
42.1858 \\
42.3403 \\
42.3401 \\
42.3200 \\
42.4032 \\
42.2706 \\
42.1716 \\
42.1713 \\
42.1647 \\
42.1905 \\
42.4538 \\
42.1964 \\
42.1971 \\
42.3259 \\
42.3225 \\
42.4056 \\
42.3985 \\
42.3395 \\
42.3639 \\
42.3250 \\
42.2176 \\
42.2229 \\
42.2532 \\
42.2512 \\
42.3283 \\
42.3626 \\
42.4373 \\
42.2557 \\
42.2295 \\
42.3283 \\
42.2561 \\
42.8456 \\
42.2036 \\
42.3585\end{array}$ & $\begin{array}{l}79.6860 \\
79.6781 \\
79.2917 \\
79.3682 \\
79.6357 \\
78.8924 \\
78.8511 \\
78.8511 \\
79.1920 \\
79.1920 \\
79.3150 \\
79.7029 \\
79.3991 \\
79.7009 \\
79.4799 \\
79.4718 \\
77.5427 \\
79.6990 \\
79.4156 \\
79.3861 \\
78.9841 \\
79.6676 \\
79.4218 \\
79.3958 \\
79.3492 \\
79.2938 \\
77.6400 \\
79.7096 \\
79.7189 \\
79.7221 \\
79.7098 \\
77.6231 \\
79.6975 \\
79.7091 \\
79.3422 \\
79.3756 \\
78.8784 \\
78.8738 \\
79.4975 \\
79.3117 \\
79.3671 \\
79.6389 \\
79.6255 \\
79.6288 \\
79.6210 \\
79.3575 \\
79.4998 \\
79.2936 \\
79.6389 \\
79.6361 \\
79.3780 \\
79.6154 \\
78.8337 \\
79.6537 \\
79.5036\end{array}$ & $\begin{array}{l}472.4 \\
484.6 \\
424.6 \\
399.0 \\
487.1 \\
390.1 \\
451.1 \\
451.1 \\
423.7 \\
423.7 \\
271.3 \\
460.9 \\
406.6 \\
457.8 \\
442.9 \\
436.2 \\
646.2 \\
449.0 \\
416.7 \\
398.7 \\
243.8 \\
445.0 \\
420.0 \\
398.7 \\
469.4 \\
329.2 \\
473.4 \\
438.9 \\
438.9 \\
442.3 \\
447.4 \\
538.0 \\
445.0 \\
439.8 \\
504.4 \\
457.8 \\
435.9 \\
435.9 \\
321.0 \\
405.7 \\
400.5 \\
511.8 \\
513.0 \\
481.0 \\
477.9 \\
438.3 \\
235.0 \\
262.1 \\
458.4 \\
489.2 \\
419.7 \\
465.7 \\
176.8 \\
481.9 \\
232.9\end{array}$ & $\begin{array}{l}506.0 \\
488.3 \\
427.0 \\
402.0 \\
487.4 \\
393.2 \\
454.2 \\
454.2 \\
424.6 \\
424.6 \\
274.6 \\
463.9 \\
409.0 \\
460.9 \\
444.4 \\
438.3 \\
649.8 \\
452.0 \\
416.7 \\
401.1 \\
246.9 \\
448.1 \\
422.5 \\
401.7 \\
472.1 \\
332.5 \\
477.0 \\
442.0 \\
442.0 \\
445.3 \\
450.5 \\
541.6 \\
448.1 \\
442.9 \\
507.5 \\
460.2 \\
438.3 \\
438.3 \\
323.4 \\
408.1 \\
402.9 \\
514.9 \\
515.4 \\
483.7 \\
481.0 \\
440.7 \\
237.1 \\
265.2 \\
461.5 \\
494.4 \\
423.44 \\
465.7 \\
176.8 \\
484.9 \\
235.0\end{array}$ & $\begin{array}{r}1139.6 \\
1121.1 \\
984.5 \\
1004.3 \\
1089.7 \\
1001.0 \\
1055.2 \\
1055.2 \\
607.2 \\
603.8 \\
763.8 \\
1138.4 \\
993.6 \\
1107.0 \\
1000.0 \\
999.7 \\
1375.6 \\
1107.3 \\
992.4 \\
958.3 \\
1469.7 \\
1082.0 \\
990.0 \\
969.9 \\
1076.6 \\
853.4 \\
1118.6 \\
1091.2 \\
1090.6 \\
1095.8 \\
1078.7 \\
970.5 \\
1077.8 \\
1077.8 \\
1150.0 \\
1029.3 \\
1051.0 \\
1050.6 \\
866.2 \\
962.3 \\
1208.5 \\
1134.2 \\
1132.9 \\
1083.0 \\
1089.7 \\
1026.3 \\
756.3 \\
731.5 \\
1051.0 \\
1105.2 \\
997.6 \\
1079.6 \\
3122.8 \\
752.3 \\
10.9\end{array}$ & $\begin{array}{l}35.0 \\
34.4 \\
32.2 \\
32.8 \\
34.4 \\
32.2 \\
33.9 \\
35.0 \\
21.1 \\
21.1 \\
24.4 \\
35.0 \\
31.1 \\
31.7 \\
31.1 \\
31.1 \\
43.3 \\
32.8 \\
32.2 \\
32.8 \\
39.4 \\
32.2 \\
32.8 \\
32.8 \\
32.8 \\
25.6 \\
32.8 \\
32.2 \\
32.8 \\
32.8 \\
32.2 \\
29.4 \\
32.2 \\
33.9 \\
31.7 \\
32.2 \\
32.2 \\
32.8 \\
30.6 \\
29.4 \\
31.7 \\
36.1 \\
33.3 \\
32.2 \\
35.0 \\
36.7 \\
28.9 \\
24.4 \\
33.3 \\
35.6 \\
33.9 \\
35.0 \\
23.3 \\
36.1 \\
26.7\end{array}$ & $\begin{array}{l}7.6 \\
7.7 \\
7.9 \\
8.3 \\
7.6 \\
8.1 \\
7.5 \\
7.5 \\
7.9 \\
7.9 \\
9.3 \\
8.1 \\
8.2 \\
8.0 \\
7.8 \\
7.9 \\
5.9 \\
8.2 \\
8.1 \\
8.2 \\
9.1\end{array}$ & $\begin{array}{l}24.0 \\
23.8 \\
24.7 \\
24.4 \\
24.6 \\
24.1 \\
25.0 \\
26.1 \\
21.8 \\
21.9 \\
19.8 \\
23.6 \\
23.1 \\
21.4 \\
23.3 \\
23.2 \\
27.2 \\
22.2 \\
24.3 \\
25.6 \\
20.6 \\
22.3 \\
25.0 \\
25.3 \\
23.4 \\
19.7 \\
22.6 \\
22.0 \\
22.5 \\
22.4 \\
22.4 \\
23.6 \\
22.4 \\
23.9 \\
21.3 \\
23.8 \\
23.3 \\
23.9 \\
24.9 \\
22.2 \\
19.4 \\
25.3 \\
22.9 \\
22.7 \\
25.1 \\
28.1 \\
25.3 \\
20.7 \\
24.3 \\
25.3 \\
25.9 \\
25.2 \\
40.6 \\
25.3 \\
22.4\end{array}$ & $\begin{array}{l}6.9 \\
6.8 \\
5.8 \\
6.0 \\
6.5 \\
5.9 \\
6.3 \\
6.3 \\
3.2 \\
3.2 \\
4.3 \\
6.9 \\
5.9 \\
6.7 \\
5.9 \\
5.9 \\
8.5 \\
6.7 \\
5.9 \\
5.6 \\
9.2 \\
6.5 \\
5.9 \\
5.7 \\
6.5 \\
4.9 \\
6.8 \\
6.6 \\
6.6 \\
6.6 \\
6.5 \\
5.7 \\
6.5 \\
6.5\end{array}$ & $\begin{array}{l}30.1 \\
29.9 \\
30.6 \\
30.3 \\
30.6 \\
30.0 \\
31.0 \\
32.0 \\
27.0 \\
27.1 \\
25.4 \\
29.7 \\
29.0 \\
27.4 \\
29.2 \\
29.1 \\
33.5 \\
28.2 \\
30.2 \\
31.5 \\
26.9 \\
28.3 \\
30.9 \\
31.2 \\
29.4 \\
25.4 \\
28.7 \\
28.0 \\
28.5 \\
28.4 \\
28.4\end{array}$ \\
\hline
\end{tabular}


APPENDIX A (Cont)

\begin{tabular}{|c|c|c|c|c|c|c|c|c|c|c|c|c|}
\hline $\begin{array}{l}\text { NELL } \\
\text { NO }\end{array}$ & & $\begin{array}{l}\text { DATE } \\
\text { LOG }\end{array}$ & $\begin{array}{l}\text { ATITUDE } \\
\text { DEGREES }\end{array}$ & $\begin{array}{l}\text { NGG ITUDE } \\
\text { EGREES }\end{array}$ & $\underset{\mathbf{H}}{\text { ELEV }}$ & BELV & EPTH & $\begin{array}{c}\text { BHT } \\
\text { C }\end{array}$ & $\begin{array}{l}\text { REMP } \\
\text { C }\end{array}$ & $\begin{array}{l}\text { GRADIENT } \\
\mathrm{C} / \mathrm{KM}\end{array}$ & $\underset{\mathrm{C}}{\mathrm{BTCOR}}$ & $\begin{array}{l}\text { AAPGRA } \\
\text { C/KM }\end{array}$ \\
\hline $\begin{array}{l}11197 \\
11199 \\
11200 \\
11205 \\
11207 \\
11208 \\
11210 \\
11211 \\
11212 \\
11254 \\
11255 \\
11256 \\
11257 \\
11258 \\
11262 \\
11263 \\
11265 \\
11267 \\
11268 \\
11269 \\
11273 \\
11274 \\
11275 \\
11285 \\
11294 \\
11295 \\
11296 \\
11297 \\
11298 \\
11304 \\
11305 \\
11310 \\
11311 \\
11312 \\
11314 \\
11315 \\
11316 \\
11323 \\
11337 \\
11339 \\
11340 \\
11341 \\
11342 \\
11349 \\
11350 \\
11352 \\
11353 \\
11354 \\
11355 \\
11358 \\
11362 \\
11363 \\
11364 \\
11370 \\
11372 \\
\end{array}$ & 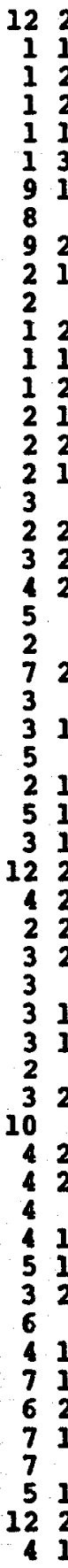 & $\begin{array}{rl}22 & 74 \\
17 & 75 \\
29 & 75 \\
20 & 75 \\
14 & 75 \\
31 & 75 \\
10 & 75 \\
6 & 75 \\
29 & 75 \\
12 & 75 \\
8 & 75 \\
28 & 75 \\
16 & 75 \\
27 & 75 \\
19 & 75 \\
27 & 75 \\
18 & 75 \\
8 & 75 \\
24 & 75 \\
25 & 75 \\
27 & 75 \\
5 & 75 \\
9 & 75 \\
25 & 75 \\
7 & 75 \\
16 & 75 \\
5 & 75 \\
16 & 75 \\
19 & 75 \\
16 & 75 \\
20 & 75 \\
29 & 75 \\
27 & 75 \\
20 & 75 \\
9 & 75 \\
10 & 75 \\
17 & 75 \\
7 & 75 \\
20 & 75 \\
2 & 75 \\
21 & 75 \\
28 & 75 \\
3 & 75 \\
18 & 75 \\
14 & 75 \\
26 & 75 \\
2 & 75 \\
10 & 75 \\
10 & 75 \\
27 & 76 \\
12 & 75 \\
3 & 75 \\
12 & 75 \\
23 & 75 \\
12 & 75\end{array}$ & $\begin{array}{l}42.2023 \\
42.2051 \\
42.2467 \\
42.3324 \\
42.3533 \\
42.3500 \\
42.1004 \\
42.1008 \\
42.0946 \\
42.2278 \\
42.2598 \\
42.2337 \\
42.2643 \\
42.2689 \\
42.3648 \\
42.3589 \\
42.4177 \\
42.3247 \\
42.3195 \\
42.2604 \\
42.3351 \\
42.3371 \\
42.3451 \\
42.1070 \\
42.3207 \\
42.2628 \\
42.3145 \\
42.3199 \\
42.2667 \\
42.3285 \\
42.3240 \\
42.4065 \\
42.7552 \\
42.8119 \\
42.2740 \\
42.3343 \\
42.3248 \\
42.3329 \\
42.3264 \\
42.3357 \\
42.3062 \\
42.2991 \\
42.3149 \\
42.3288 \\
42.3241 \\
42.3291 \\
42.3241 \\
42.3133 \\
42.3328 \\
42.4251 \\
42.2132 \\
42.2182 \\
42.2733 \\
42.4155 \\
42.3092\end{array}$ & $\begin{array}{l}79.6696 \\
79.6273 \\
79.6167 \\
79.3448 \\
79.5083 \\
79.5166 \\
79.4640 \\
79.4553 \\
79.4635 \\
79.6272 \\
79.6322 \\
79.6242 \\
79.6568 \\
79.6491 \\
79.5063 \\
79.5136 \\
79.3718 \\
79.4507 \\
79.4073 \\
79.6904 \\
79.5033 \\
79.5120 \\
79.5038 \\
79.4583 \\
79.4158 \\
79.6828 \\
79.3985 \\
79.3892 \\
79.6877 \\
79.4443 \\
79.4363 \\
79.3890 \\
78.3475 \\
78.1939 \\
79.6242 \\
79.4924 \\
79.4213 \\
79.3835 \\
79.4893 \\
79.4296 \\
79.3562 \\
79.3542 \\
79.3544 \\
79.5062 \\
79.5104 \\
79.3329 \\
79.5003 \\
79.4953 \\
79.4383 \\
79.3485 \\
79.7015 \\
79.6983 \\
79.6904 \\
79.2932 \\
79.3510\end{array}$ & $\begin{array}{l}471.8 \\
483.7 \\
478.5 \\
511.1 \\
239.0 \\
238.0 \\
478.5 \\
467.3 \\
468.5 \\
483.7 \\
470.0 \\
485.2 \\
408.4 \\
416.1 \\
216.1 \\
226.2 \\
237.7 \\
485.2 \\
442.6 \\
296.3 \\
429.8 \\
294.7 \\
271.0 \\
483.1 \\
438.9 \\
310.9 \\
442.6 \\
406.0 \\
262.7 \\
467.0 \\
449.9 \\
239.3 \\
539.5 \\
498.3 \\
442.9 \\
377.3 \\
424.3 \\
421.5 \\
437.4 \\
417.0 \\
394.1 \\
395.6 \\
405.4 \\
354.5 \\
359.4 \\
494.1 \\
411.8 \\
415.1 \\
445.6 \\
241.4 \\
425.2 \\
419.7 \\
232.3 \\
295.7 \\
411.5\end{array}$ & $\begin{array}{l}474.9 \\
486.2 \\
481.6 \\
513.6 \\
241.1 \\
240.2 \\
481.9 \\
470.6 \\
471.8 \\
486.2 \\
473.0 \\
487.7 \\
411.5 \\
419.1 \\
218.2 \\
228.6 \\
240.5 \\
487.7 \\
445.0 \\
299.3 \\
431.9 \\
296.9 \\
273.1 \\
486.5 \\
441.4 \\
313.9 \\
445.0 \\
408.4 \\
265.8 \\
469.4 \\
452.0 \\
242.9 \\
542.5 \\
501.4 \\
445.9 \\
379.5 \\
426.7 \\
424.0 \\
439.5 \\
420.0 \\
396.5 \\
395.6 \\
407.8 \\
356.6 \\
361.5 \\
496.5 \\
413.9 \\
417.0 \\
447.8 \\
241.4 \\
428.2 \\
422.8 \\
235.3 \\
296.6 \\
413.9 \\
\end{array}$ & $\begin{array}{r}1097.3 \\
1091.8 \\
1092.1 \\
1090.6 \\
772.7 \\
792.8 \\
1244.2 \\
1040.9 \\
1240.5 \\
1103.7 \\
1067.7 \\
1100.9 \\
985.4 \\
992.7 \\
738.2 \\
750.1 \\
716.3 \\
1051.6 \\
1028.4 \\
1174.4 \\
895.8 \\
834.8 \\
810.5 \\
1259.1 \\
1020.2 \\
878.7 \\
1034.5 \\
989.1 \\
824.2 \\
1033.9 \\
1016.2 \\
737.3 \\
872.0 \\
815.3 \\
1011.6 \\
930.9 \\
1026.3 \\
999.7 \\
1000.4 \\
982.7 \\
1003.4 \\
1006.4 \\
1002.2 \\
910.1 \\
941.2 \\
1083.6 \\
977.2 \\
982.4 \\
1013.2 \\
723.9 \\
1020.8 \\
1033.9 \\
794.3 \\
780.0 \\
1014.4\end{array}$ & $\begin{array}{l}32.8 \\
35.6 \\
35.6 \\
33.3 \\
28.3 \\
28.9 \\
36.1 \\
33.3 \\
33.3 \\
35.6 \\
35.0 \\
35.0 \\
31.7 \\
33.3 \\
29.4 \\
29.4 \\
22.8 \\
32.2 \\
31.1\end{array}$ & $\begin{array}{r}7.8 \\
7.7 \\
7.7 \\
7.1 \\
9.8 \\
9.8 \\
8.0 \\
8.1 \\
8.1 \\
7.7 \\
7.7 \\
7.6 \\
8.3 \\
8.2 \\
10.0 \\
9.9 \\
9.6 \\
7.4 \\
7.8 \\
9.4 \\
7.9 \\
9.3 \\
9.5 \\
7.9 \\
7.9 \\
9.3 \\
7.9 \\
8.2 \\
9.7 \\
7.6 \\
7.8\end{array}$ & $\begin{array}{l}22.7 \\
25.5 \\
25.5 \\
24.0 \\
24.0 \\
24.1 \\
22.6 \\
24.2 \\
20.3 \\
25.3 \\
25.5 \\
24.9 \\
23.7 \\
25.3 \\
26.4 \\
26.1 \\
18.4 \\
23.6 \\
22.6 \\
18.5 \\
25.2 \\
24.2 \\
26.0 \\
23.7 \\
23.3 \\
23.0 \\
22.5 \\
22.0 \\
25.9 \\
22.8 \\
21.9 \\
17.1 \\
25.7 \\
15.6 \\
21.2 \\
21.4 \\
26.3 \\
25.3 \\
32.1 \\
24.0 \\
22.7 \\
27.6 \\
25.1 \\
25.2 \\
25.6 \\
21.4 \\
22.9 \\
25.1 \\
25.2 \\
27.4 \\
25.1 \\
25.8 \\
23.8 \\
26.1 \\
23.2\end{array}$ & $\begin{array}{l}5 \\
4 \\
4 \\
7 \\
6 \\
5 \\
6 \\
5 \\
4 \\
6 \\
6 \\
4 \\
5 \\
4 \\
6 \\
5 \\
6 \\
5 \\
5 \\
5 \\
5 \\
6 \\
5 \\
5 \\
5 \\
6 \\
5 \\
5 \\
6\end{array}$ & $\begin{array}{l}8.7 \\
1.5 \\
1.5 \\
0.0 \\
9.6 \\
9.7 \\
8.7 \\
0.2 \\
6.5 \\
1.3 \\
1.5 \\
0.9 \\
9.6 \\
1.2 \\
1.9 \\
3.7 \\
3.9\end{array}$ \\
\hline
\end{tabular}


APPENDIX A (Cont)

\begin{tabular}{|c|c|c|c|c|c|c|c|c|c|c|c|c|}
\hline $\begin{array}{l}\text { WELL } \\
\text { NO }\end{array}$ & & $\begin{array}{l}\text { DATE } \\
\text { LOG }\end{array}$ & $\begin{array}{c}\text { LATITUDE } \\
\text { DEGREES }\end{array}$ & $\begin{array}{l}\text { LONG ITUDE } \\
\text { DEGREES }\end{array}$ & $\underset{\mathbf{M}}{\text { ELEV }}$ & $\underset{M}{\text { KBELV }}$ & $\underset{M}{\text { DEPTH }}$ & $\begin{array}{c}\text { BHT } \\
\text { C }\end{array}$ & $\underset{C}{\text { STEMP }}$ & $\begin{array}{l}\text { GRADIENT } \\
\mathrm{C} / \mathrm{KM}\end{array}$ & $\underset{\text { C }}{\text { BHTCOR }}$ & $\begin{array}{l}\text { AAPGRAD } \\
\text { C/KM }\end{array}$ \\
\hline $\begin{array}{l}11373 \\
11387 \\
11388 \\
11403 \\
11405 \\
11406 \\
11407 \\
11414 \\
11415 \\
11422 \\
11423 \\
11424 \\
11427 \\
11428 \\
11429 \\
11430 \\
11431 \\
11447 \\
11448 \\
11453 \\
11454 \\
11455 \\
11456 \\
11457 \\
11459 \\
11462 \\
11469 \\
11470 \\
11471 \\
11472 \\
11473 \\
11477 \\
11478 \\
11480 \\
11482 \\
11486 \\
11487 \\
11488 \\
11489 \\
11499 \\
11500 \\
11501 \\
11502 \\
11503 \\
11504 \\
11505 \\
11510 \\
11511 \\
11512 \\
11513 \\
11524 \\
11525 \\
11526 \\
11530 \\
11532\end{array}$ & $\begin{array}{r}8 \\
6 \\
10\end{array}$ & $\begin{array}{rl}11 & 75 \\
1 & 75 \\
22 & 75 \\
1 & 75 \\
24 & 75 \\
1 & 75 \\
3 & 75 \\
25 & 75 \\
19 & 75 \\
23 & 75 \\
2 & 75 \\
15 & 75 \\
8 & 75 \\
26 & 75 \\
26 & 75 \\
19 & 75 \\
30 & 75 \\
14 & 75 \\
9 & 75 \\
1 & 75 \\
11 & 76 \\
12 & 75 \\
1 & 75 \\
7 & 75 \\
15 & 75 \\
4 & 75 \\
23 & 75 \\
11 & 75 \\
24 & 75 \\
29 & 75 \\
10 & 75 \\
20 & 75 \\
12 & 75 \\
2 & 75 \\
16 & 75 \\
23 & 75 \\
15 & 75 \\
2 & 75 \\
23 & 75 \\
19 & 75 \\
27 & 75 \\
15 & 75 \\
25 & 75 \\
3 & 75 \\
20 & 75 \\
12 & 75 \\
14 & 75 \\
22 & 75 \\
8 & 75 \\
30 & 75 \\
10 & 75 \\
19 & 75 \\
16 & 75 \\
22 & 75 \\
16 & 75\end{array}$ & $\begin{array}{l}42.3276 \\
42.2214 \\
42.1058 \\
42.7258 \\
42.3240 \\
42.3195 \\
42.2083 \\
42.1971 \\
42.3154 \\
42.3951 \\
42.3920 \\
42.3125 \\
42.3070 \\
42.8972 \\
42.2691 \\
42.3331 \\
42.3094 \\
42.8783 \\
42.3928 \\
42.3925 \\
42.4115 \\
42.3215 \\
42.3251 \\
42.3096 \\
42.2235 \\
42.2069 \\
42.4498 \\
42.4543 \\
42.4526 \\
42.4463 \\
42.4416 \\
42.2382 \\
42.4735 \\
42.2038 \\
42.3129 \\
42.3191 \\
42.3062 \\
42.3239 \\
42.2988 \\
42.3677 \\
42.2596 \\
42.3123 \\
42.3654 \\
42.3605 \\
42.3648 \\
42.3664 \\
42.5869 \\
42.3986 \\
42.4380 \\
42.3115 \\
42.3184 \\
42.3281 \\
42.3949 \\
42.1143 \\
42.3741\end{array}$ & $\begin{array}{l}79.3230 \\
79.6666 \\
79.4780 \\
77.9534 \\
79.3157 \\
79.3104 \\
79.6944 \\
79.6499 \\
79.3439 \\
79.4102 \\
79.3532 \\
79.4075 \\
79.3835 \\
77.1142 \\
79.6424 \\
79.5240 \\
79.3933 \\
78.8109 \\
79.3438 \\
79.4028 \\
79.3555 \\
79.4843 \\
79.5204 \\
79.5063 \\
79.6419 \\
77.2508 \\
79.2962 \\
79.3028 \\
79.2828 \\
79.2896 \\
79.2517 \\
79.6276 \\
78.4283 \\
78.8767 \\
79.3704 \\
79.4750 \\
79.3666 \\
79.4671 \\
79.3675 \\
79.2750 \\
79.6986 \\
79.3037 \\
79.2669 \\
79.2597 \\
79.2530 \\
79.3181 \\
78.7008 \\
79.3789 \\
79.2701 \\
79.5141 \\
79.5193 \\
79.4965 \\
79.3651 \\
79.46996 \\
79.4345\end{array}$ & $\begin{array}{l}458.7 \\
493.2 \\
466.3 \\
334.1 \\
410.3 \\
399.6 \\
443.5 \\
470.9 \\
490.7 \\
248.4 \\
365.8 \\
458.1 \\
441.0 \\
242.3 \\
414.5 \\
273.4 \\
447.4 \\
182.3 \\
376.4 \\
252.4 \\
265.2 \\
429.8 \\
327.4 \\
397.8 \\
520.0 \\
462.1 \\
244.8 \\
231.0 \\
251.2 \\
255.4 \\
356.6 \\
479.8 \\
531.6 \\
555.7 \\
409.7 \\
458.7 \\
395.0 \\
445.9 \\
394.4 \\
450.2 \\
269.7 \\
398.4 \\
459.3 \\
473.4 \\
474.9 \\
402.3 \\
325.2 \\
283.2 \\
339.9 \\
374.0 \\
371.9 \\
421.2 \\
316.1 \\
519.7 \\
256.6\end{array}$ & $\begin{array}{l}461.2 \\
496.2 \\
469.7 \\
337.4 \\
412.7 \\
402.0 \\
446.5 \\
474.0 \\
493.0 \\
252.1 \\
369.1 \\
460.6 \\
443.5 \\
245.1 \\
417.6 \\
275.5 \\
449.9 \\
185.6 \\
379.8 \\
255.4 \\
268.2 \\
432.2 \\
329.5 \\
399.9 \\
523.0 \\
462.1 \\
247.8 \\
234.4 \\
254.5 \\
259.1 \\
359.7 \\
482.8 \\
534.9 \\
559.0 \\
412.1 \\
460.9 \\
397.5 \\
448.1 \\
396.8 \\
453.2 \\
272.8 \\
401.4 \\
462.4 \\
476.4 \\
477.3 \\
404.8 \\
328.6 \\
286.5 \\
342.6 \\
376.4 \\
374.0 \\
423.4 \\
319.1 \\
523.0 \\
259.7\end{array}$ & 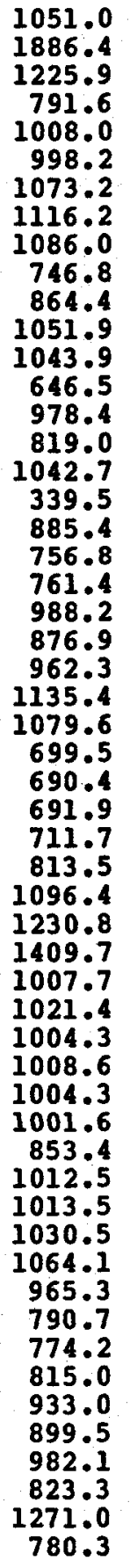 & $\begin{array}{l}33.9 \\
47.2 \\
33.3 \\
30.0 \\
33.3 \\
33.9 \\
33.3 \\
34.4 \\
33.3 \\
32.2 \\
26.1 \\
34.4 \\
32.2 \\
30.0 \\
32.2 \\
31.1 \\
33.9 \\
23.3 \\
31.7 \\
24.4 \\
29.4 \\
32.2 \\
30.6 \\
34.4 \\
35.6 \\
27.8 \\
22.2 \\
23.3 \\
25.6 \\
28.3 \\
24.4 \\
34.4 \\
35.6 \\
42.2 \\
33.9 \\
35.0 \\
33.3 \\
33.9 \\
33.3 \\
31.7 \\
31.1 \\
32.2 \\
34.4 \\
31.7 \\
32.2 \\
30.6 \\
25.0 \\
35.6 \\
28.3 \\
32.2 \\
32.2 \\
31.7 \\
26.7 \\
36.7 \\
28.9\end{array}$ & $\begin{array}{l}7.7 \\
7.6 \\
8.1 \\
8.0 \\
8.1 \\
8.3 \\
8.1 \\
7.9 \\
7.4 \\
9.6 \\
8.4 \\
7.7 \\
7.9 \\
8.5 \\
8.2 \\
9.5 \\
7.8 \\
9.0 \\
8.3 \\
9.5 \\
9.4 \\
8.0 \\
9.0 \\
8.3 \\
7.3 \\
7.7 \\
9.5 \\
9.6 \\
9.4 \\
9.4 \\
8.4 \\
7.7 \\
6.6 \\
7.0 \\
8.2 \\
7.7 \\
8.3 \\
7.8 \\
8.4\end{array}$ & $\begin{array}{l}25.0 \\
21.0 \\
20.6 \\
27.8 \\
25.0 \\
25.7 \\
23.5 \\
23.8 \\
23.9 \\
30.4 \\
20.5 \\
25.4 \\
23.3 \\
33.2 \\
24.5 \\
26.4 \\
25.0 \\
42.1 \\
26.4 \\
19.7 \\
26.4 \\
24.5 \\
24.6 \\
27.2 \\
24.9 \\
18.6 \\
18.2 \\
19.9 \\
23.4 \\
26.7 \\
19.7 \\
24.4 \\
23.5 \\
25.0 \\
25.5 \\
26.7 \\
24.9 \\
25.9 \\
24.9 \\
24.0 \\
25.1 \\
23.6 \\
26.5 \\
23.5 \\
23.3 \\
23.2 \\
21.0 \\
34.0 \\
24.3 \\
25.4 \\
26.3 \\
24.1 \\
21.6 \\
22.9 \\
24.8\end{array}$ & $\begin{array}{r}6.3 \\
12.1 \\
7.5 \\
4.5 \\
6.0 \\
5.9 \\
6.4 \\
6.7 \\
6.5 \\
4.2 \\
5.0 \\
6.3 \\
6.2 \\
3.5 \\
5.8 \\
4.7 \\
6.2 \\
1.3 \\
5.1 \\
4.2 \\
4.3 \\
5.8 \\
5.1 \\
5.7 \\
6.9 \\
6.5 \\
3.8 \\
3.8 \\
3.8 \\
3.9 \\
4.6 \\
6.6 \\
7.5 \\
78 \\
8.8 \\
6.0 \\
6.1 \\
6.0 \\
6.0 \\
6.0 \\
5.9 \\
4.9 \\
6.0 \\
6.0 \\
6.1 \\
6.4 \\
5.7 \\
4.5 \\
4.3 \\
4.6 \\
5.5 \\
5.2 \\
5.8 \\
4.7 \\
77 .\end{array}$ & $\begin{array}{l}30.9 \\
27.4 \\
26.7 \\
33.5 \\
30.9 \\
31.6 \\
29.5 \\
29.8 \\
29.9 \\
35.9 \\
26.2 \\
31.4 \\
29.3 \\
38.5 \\
30.4 \\
32.1 \\
31.0 \\
45.9 \\
32.2 \\
25.3 \\
32.0 \\
30.5 \\
30.4 \\
33.0 \\
30.9 \\
24.6 \\
23.7 \\
25.4 \\
28.8 \\
32.1 \\
25.4 \\
30.4 \\
29.7 \\
31.2 \\
31.4 \\
32.7 \\
30.8 \\
31.8 \\
30.8 \\
29.9 \\
30.8 \\
29.6 \\
32.5 \\
29.5 \\
29.3 \\
29.1 \\
26.7 \\
39.6 \\
29.9 \\
31.2 \\
32.1 \\
30.0 \\
27.3 \\
29.0 \\
30.4\end{array}$ \\
\hline
\end{tabular}


APPENDIX A (Cont)

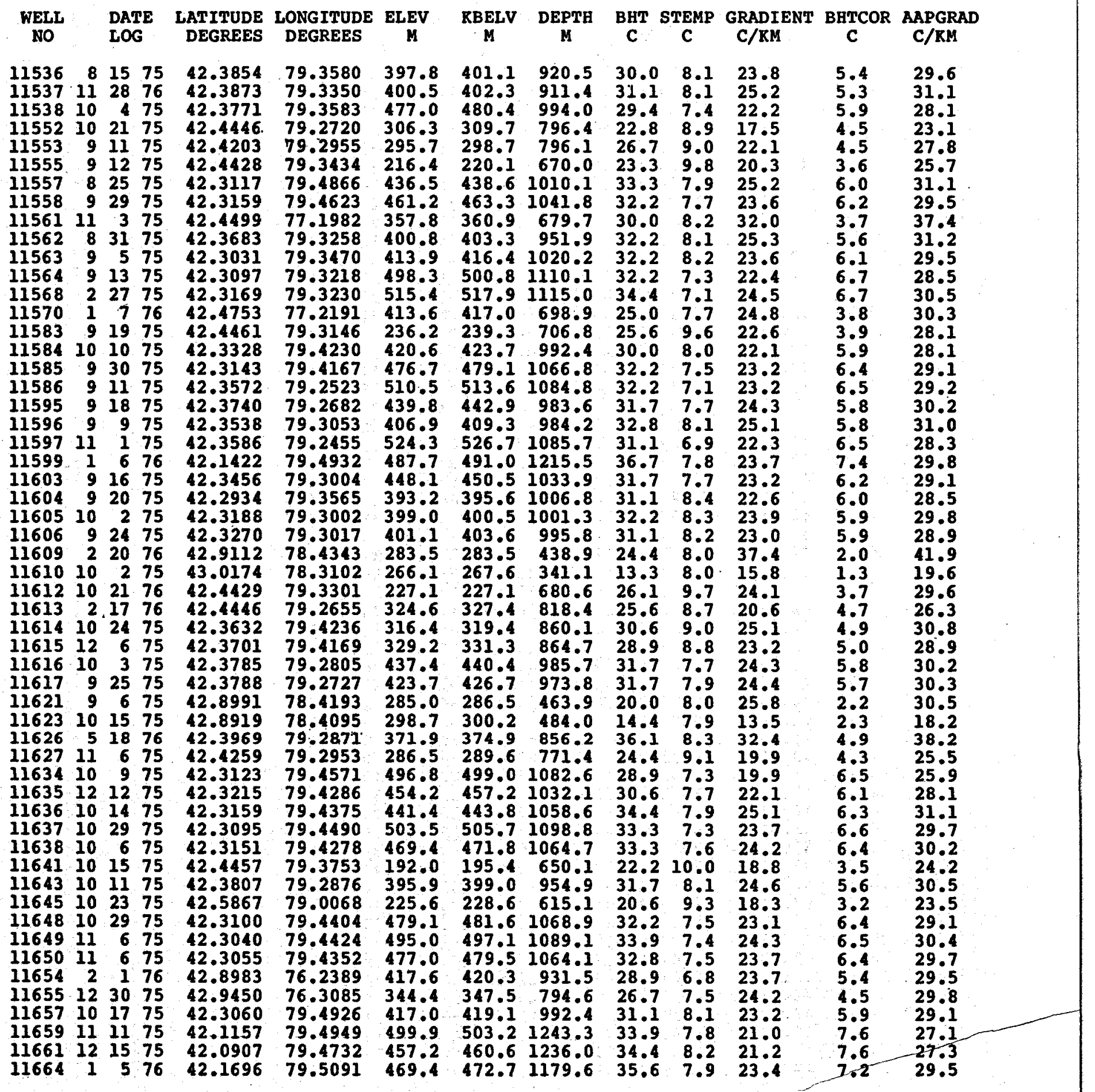


APPENDIX A (Cont)

\begin{tabular}{|c|c|c|c|c|c|c|c|c|c|c|c|c|}
\hline $\begin{array}{l}\text { WELL } \\
\text { NO }\end{array}$ & & D & $\begin{array}{c}\text { LATITUDE } \\
\text { DEGREES }\end{array}$ & $\begin{array}{l}\text { LONGITUDE } \\
\text { DEGREES }\end{array}$ & $\underset{M}{\text { ELEV }}$ & $\underset{M}{\text { RBELV }}$ & $\underset{M}{\text { DEPTH }}$ & $\begin{array}{c}\text { BHT } \\
\text { C }\end{array}$ & $\underset{C}{\text { STEMP }}$ & $\begin{array}{c}\text { GRADIENT } \\
\text { C/KM }\end{array}$ & $\underset{\mathrm{C}}{\mathrm{HTCOR}}$ & $\begin{array}{l}\text { AAPGRAD } \\
\text { C/KM }\end{array}$ \\
\hline $\begin{array}{l}11666 \\
11667 \\
11671 \\
11672 \\
11676 \\
11678 \\
11679 \\
11680 \\
11681 \\
11682 \\
11683 \\
11684 \\
11686 \\
11687 \\
11691 \\
11696 \\
11697 \\
11698 \\
11699 \\
11701 \\
11702 \\
11705 \\
11706 \\
11708 \\
11710 \\
11711 \\
11712 \\
11713 \\
11714 \\
11715 \\
11716 \\
11717 \\
11718 \\
11720 \\
11721 \\
11723 \\
11724 \\
11728 \\
11730 \\
11734 \\
11742 \\
11748 \\
11751 \\
11752 \\
11753 \\
11754 \\
11762 \\
11764 \\
11765 \\
11766 \\
11774 \\
11776 \\
11777 \\
11778 \\
11781 \\
\end{array}$ & $\begin{array}{r}5 \\
5 \\
6 \\
11 \\
12 \\
12 \\
11 \\
11 \\
11 \\
1 \\
11 \\
8 \\
11 \\
2 \\
12 \\
11 \\
1 \\
11 \\
11 \\
11 \\
11 \\
11 \\
11 \\
11 \\
11\end{array}$ & $\begin{array}{r}20 \\
30 \\
22 \\
22 \\
17 \\
5 \\
9 \\
17 \\
12 \\
24 \\
20 \\
14 \\
7 \\
2 \\
11 \\
5 \\
4 \\
13 \\
3 \\
2 \\
5 \\
17 \\
12 \\
27 \\
2 \\
13 \\
26 \\
14 \\
9 \\
27 \\
28 \\
19 \\
15 \\
20 \\
23 \\
23\end{array}$ & $\begin{array}{l}42.8452 \\
42.0663 \\
42.3103 \\
42.1615 \\
42.4505 \\
42.4356 \\
42.4539 \\
42.4273 \\
42.4342 \\
42.4302 \\
42.4458 \\
42.4402 \\
42.4417 \\
42.4461 \\
42.4561 \\
42.3041 \\
42.3207 \\
42.3165 \\
42.8784 \\
42.3745 \\
42.3884 \\
42.0316 \\
42.4500 \\
42.8386 \\
42.4859 \\
42.3593 \\
42.3459 \\
42.3341 \\
42.3679 \\
42.2971 \\
42.3033 \\
42.3053 \\
42.2900 \\
42.3544 \\
42.4446 \\
42.4690 \\
42.3884 \\
42.5695 \\
42.7142 \\
42.1161 \\
42.8870 \\
41.9458 \\
42.3384 \\
42.2944 \\
42.3847 \\
42.3668 \\
42.1599 \\
42.3807 \\
42.2985 \\
42.3036 \\
42.4415 \\
42.8968 \\
42.9007 \\
42.8992 \\
42.3056\end{array}$ & $\begin{array}{l}76.7878 \\
79.7366 \\
79.4237 \\
79.4705 \\
79.3678 \\
79.3623 \\
79.3656 \\
79.3719 \\
79.3737 \\
79.3538 \\
79.2331 \\
79.2335 \\
79.1992 \\
79.1941 \\
79.2597 \\
79.4615 \\
79.4425 \\
79.4475 \\
78.4251 \\
79.2864 \\
79.2949 \\
79.6815 \\
78.7951 \\
76.7793 \\
79.3261 \\
79.4172 \\
79.4239 \\
79.3139 \\
79.3061 \\
79.4040 \\
79.3896 \\
79.3329 \\
79.3474 \\
79.2604 \\
79.3497 \\
78.6807 \\
79.3688 \\
78.7350 \\
78.5182 \\
79.5186 \\
78.4210 \\
78.4047 \\
79.3071 \\
79.4161 \\
79.3754 \\
77.7259 \\
78.0128 \\
79.3881 \\
79.4424 \\
79.4519 \\
79.3580 \\
78.4080 \\
78.4038 \\
78.3975 \\
77.1636\end{array}$ & $\begin{array}{l}172.8 \\
429.8 \\
484.6 \\
477.9 \\
196.6 \\
207.3 \\
192.0 \\
220.1 \\
208.8 \\
228.6 \\
381.6 \\
402.3 \\
342.9 \\
336.8 \\
277.4 \\
481.3 \\
486.8 \\
503.5 \\
289.6 \\
430.7 \\
323.1 \\
435.9 \\
341.4 \\
175.0 \\
182.9 \\
345.9 \\
418.2 \\
395.9 \\
393.2 \\
504.4 \\
449.0 \\
463.6 \\
399.9 \\
484.9 \\
204.2 \\
417.6 \\
369.4 \\
506.3 \\
428.9 \\
554.7 \\
320.0 \\
478.5 \\
404.8 \\
499.9 \\
393.2 \\
545.6 \\
611.7 \\
390.8 \\
498.3 \\
509.0 \\
205.7 \\
295.7 \\
303.3 \\
294.1 \\
533.4\end{array}$ & $\begin{array}{l}176.2 \\
433.4 \\
487.1 \\
481.3 \\
199.9 \\
210.6 \\
195.4 \\
223.1 \\
211.2 \\
231.6 \\
384.7 \\
405.4 \\
345.9 \\
339.9 \\
280.7 \\
483.7 \\
489.8 \\
506.0 \\
291.4 \\
433.7 \\
326.1 \\
439.2 \\
341.4 \\
178.3 \\
185.9 \\
349.0 \\
420.3 \\
398.7 \\
396.2 \\
506.9 \\
450.8 \\
466.6 \\
402.0 \\
486.8 \\
207.6 \\
420.6 \\
371.6 \\
509.6 \\
431.9 \\
558.1 \\
321.9 \\
478.5 \\
406.9 \\
504.7 \\
395.6 \\
548.9 \\
615.1 \\
393.5 \\
500.5 \\
511.1 \\
208.8 \\
295.7 \\
304.5 \\
295.7 \\
536.8\end{array}$ & $\begin{array}{r}641.3 \\
1176.8 \\
1080.5 \\
1196.3 \\
654.4 \\
672.7 \\
652.0 \\
706.5 \\
673.0 \\
700.4 \\
857.4 \\
885.1 \\
834.2 \\
848.9 \\
730.6 \\
1066.5 \\
1054.9 \\
1082.3 \\
511.5 \\
991.2 \\
851.3 \\
1249.4 \\
872.0 \\
661.4 \\
613.0 \\
905.3 \\
975.4 \\
986.9 \\
947.0 \\
1112.8 \\
1056.4 \\
1081.7 \\
1016.2 \\
1075.9 \\
668.4 \\
1001.6 \\
893.4 \\
962.3 \\
1688.9 \\
1293.6 \\
501.4 \\
195.1 \\
987.9 \\
1106.4 \\
911.4 \\
1056.1 \\
1361.8 \\
918.7 \\
1095.1 \\
1097.9 \\
671.2 \\
488.3 \\
490.1 \\
504.4 \\
991.8\end{array}$ & $\begin{array}{l}27.8 \\
38.9 \\
31.1 \\
28.9 \\
20.0 \\
26.7 \\
28.9 \\
28.3 \\
28.3 \\
32.2 \\
27.2 \\
31.1 \\
28.9 \\
27.8 \\
28.9 \\
31.7 \\
31.1 \\
31.7 \\
21.1 \\
30.0 \\
27.2 \\
37.8 \\
27.8 \\
30.0 \\
26.1 \\
29.4 \\
31.1 \\
30.0 \\
31.1 \\
34.4 \\
32.2 \\
31.7 \\
30.0 \\
32.8 \\
21.1 \\
32.8 \\
30.6 \\
32.2 \\
42.8 \\
37.8 \\
20.0 \\
16.1 \\
29.4 \\
33.9 \\
30.0 \\
30.6 \\
36.1 \\
30.0 \\
30.0 \\
30.6 \\
26.7 \\
21.7 \\
13.9 \\
13.3 \\
35.0\end{array}$ & $\begin{array}{r}9.3 \\
8.6 \\
7.5 \\
7.9 \\
9.9 \\
9.9 \\
10.0 \\
9.8 \\
9.9 \\
9.7 \\
8.1 \\
7.9 \\
8.5 \\
8.6 \\
9.1 \\
7.5 \\
7.4 \\
7.3 \\
8.0 \\
7.8 \\
8.8 \\
8.6 \\
8.5 \\
9.3 \\
10.0 \\
8.7 \\
8.0 \\
8.3 \\
8.2 \\
7.3 \\
7.8 \\
7.7 \\
8.3 \\
7.4 \\
9.9 \\
7.7 \\
8.4 \\
6.6 \\
7.1 \\
7.2 \\
7.7 \\
8.2 \\
8.2 \\
7.3 \\
8.2 \\
6.6 \\
6.4 \\
8.2 \\
7\end{array}$ & $\begin{array}{l}28.8 \\
25.8 \\
21.9 \\
17.6 \\
15.4 \\
25.0 \\
29.0 \\
26.3 \\
27.5 \\
32.2 \\
22.3 \\
26.2 \\
24.4 \\
22.6 \\
27.0 \\
22.7 \\
22.5 \\
22.6 \\
25.5 \\
22.4 \\
21.6 \\
23.4 \\
22.1 \\
31.3 \\
26.3 \\
22.9 \\
23.7 \\
22.0 \\
24.2 \\
24.4 \\
23.1 \\
22.2 \\
21.3 \\
23.6 \\
16.8 \\
25.0 \\
24.8 \\
26.6 \\
21.2 \\
23.6 \\
24.5 \\
40.7 \\
21.5 \\
24.0 \\
24.0 \\
22.7 \\
21.8 \\
23.7 \\
20.7 \\
21.2 \\
25.0 \\
28.1 \\
12.3 \\
10.7 \\
28.5\end{array}$ & $\begin{array}{r}3.4 \\
7.2 \\
6.5 \\
7.3 \\
3.5 \\
3.6 \\
3.5 \\
3.99 \\
3.6 \\
3.8 \\
4.9 \\
5.1 \\
4.8 \\
4.9 \\
4.0 \\
6.4 \\
6.3 \\
6.5 \\
2.55 \\
5.9 \\
4.9 \\
7.7 \\
5.0 \\
3.6 \\
3.62 \\
5.3 \\
5.8 \\
5.8 \\
5.6 \\
6.7 \\
6.3 \\
6.5 \\
6.00 \\
6.5 \\
3.6 \\
5.9 \\
5.2 \\
5.7 \\
10.7 \\
8.0 \\
2.4 \\
5.3 \\
5.8 \\
6.7 \\
5.3 \\
6.3 \\
8.5 \\
5.44 \\
6.6 \\
6.6 \\
3.6 \\
2.3 \\
2.4 \\
2.5 \\
5.9\end{array}$ & $\begin{array}{l}4.1 \\
1.8 \\
7.9 \\
3.7 \\
0.7 \\
0.4 \\
4.4 \\
1.8 \\
2.9 \\
7.7 \\
8.0 \\
2.0 \\
0.1 \\
8.4 \\
2.6 \\
8.6 \\
8.5 \\
8.6 \\
0.4 \\
8.3 \\
7.3\end{array}$ \\
\hline
\end{tabular}


APPENDIX A (Cont)

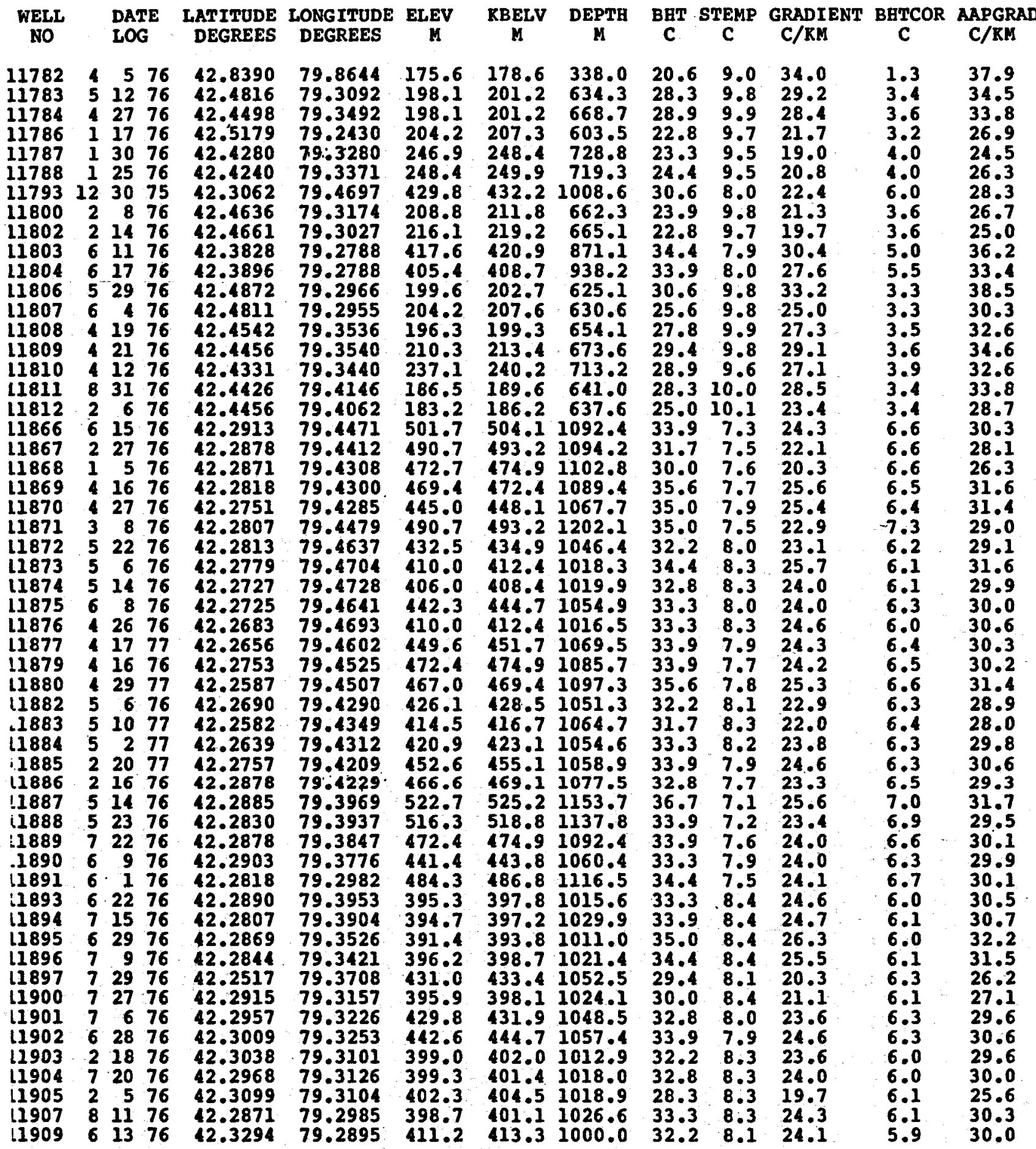


APPENDIX A (Cont)

\begin{tabular}{|c|c|c|c|c|c|c|c|c|c|c|c|c|c|}
\hline $\begin{array}{l}\text { ELL } \\
\text { NO }\end{array}$ & & & & $\begin{array}{l}\text { TTITUDE } \\
\text { EGREES }\end{array}$ & $\begin{array}{l}\text { LONGITUDE } \\
\text { DEGREES }\end{array}$ & $\begin{array}{l}\text { LEV } \\
\mathbf{M}\end{array}$ & $\begin{array}{c}\text { BELV } \\
M\end{array}$ & EPTH & $\begin{array}{c}\mathrm{BHT} \\
\mathrm{C}\end{array}$ & CEMP & $\begin{array}{l}\text { GRADIENT } \\
\text { C/KM }\end{array}$ & $\begin{array}{l}\mathrm{HTCOR} \\
\mathrm{C}\end{array}$ & $\begin{array}{l}\text { AAPGR } \\
\text { C/KM }\end{array}$ \\
\hline $\begin{array}{l}934 \\
939 \\
940 \\
941 \\
946 \\
950 \\
951 \\
952 \\
956 \\
961 \\
975 \\
977 \\
978 \\
983 \\
984\end{array}$ & & $\begin{array}{l}2 \\
2 \\
2\end{array}$ & & $\begin{array}{l}42.3269 \\
42.3225 \\
42.3165 \\
42.3089 \\
42.3115 \\
42.3063 \\
42.4427 \\
42.4516 \\
42.3328 \\
42.4419 \\
42.4405 \\
42.4656 \\
42.4654 \\
42.0748 \\
42.9398 \\
42.3003 \\
42.2057 \\
42.3009 \\
42.2937 \\
42.9642 \\
43.2928 \\
42.5511 \\
42.4484 \\
42.5031 \\
42.4490 \\
42.5578 \\
42.2114 \\
42.3123 \\
42.2235 \\
42.4567 \\
42.4606 \\
42.4606 \\
42.6775 \\
42.1362 \\
42.1667 \\
42.4342 \\
42.8481 \\
42.8545 \\
42.8580 \\
42.9318 \\
42.4494 \\
42.4880 \\
42.4891 \\
42.0593 \\
42.4836 \\
42.4846 \\
42.4786 \\
42.4786 \\
42.4772 \\
42.4732 \\
42.4556 \\
42.4502 \\
42.4495 \\
42.3617 \\
42.4655\end{array}$ & 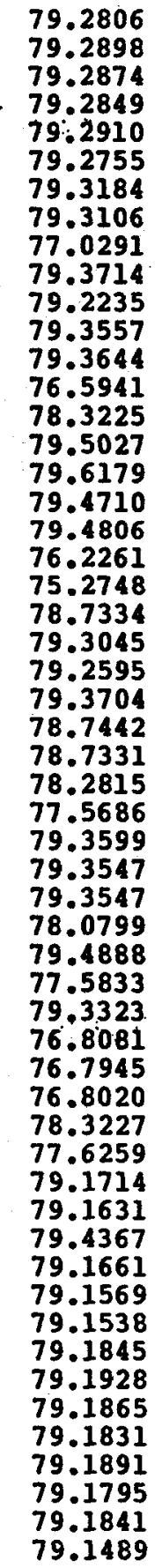 & $\begin{array}{l}411.8 \\
436.5 \\
421.2 \\
467.0 \\
237.7 \\
230.1 \\
446.5 \\
197.5 \\
408.4 \\
190.5 \\
182.9 \\
393.2 \\
287.4 \\
406.9 \\
512.1 \\
415.1 \\
409.3 \\
291.1\end{array}$ & $\begin{array}{l}453.5 \\
410.6 \\
413.9 \\
439.5 \\
423.4 \\
470.0 \\
240.5 \\
233.02 \\
449.9 \\
200.6 \\
411.5 \\
199 . .6 \\
185.9 \\
396.5 \\
-289.0 \\
409.3 \\
515.4 \\
417.6 \\
411.8 \\
293.8 \\
266.4 \\
465.7 \\
240.8 \\
204.2 \\
196.3 \\
451.1 \\
434.3 \\
652.6 \\
444.7 \\
194.8 \\
193.2 \\
193.2 \\
477.9 \\
512.4 \\
705.0 \\
234.7 \\
167.3 \\
240.2 \\
170.4 \\
282.2 \\
535.8 \\
257.9 \\
253.9 \\
216.4 \\
261.2 \\
264.9 \\
316.4 \\
296.3 \\
297.2 \\
321.6 \\
327.1 \\
300.8 \\
365.8 \\
362.9\end{array}$ & $\begin{array}{r}101 \\
122 \\
59 \\
70 \\
81 \\
68 \\
67 \\
43 \\
97 \\
70 \\
71 \\
72 \\
71 \\
72 \\
78 \\
74 \\
73 \\
78 \\
81 \\
79 \\
85 \\
86 \\
85\end{array}$ & $\begin{array}{l}32.8 \\
33.9 \\
32.2 \\
33.3 \\
31.7 \\
33.9 \\
23.9 \\
28.9 \\
28.3 \\
30.6 \\
30.0 \\
27.8 \\
28.3 \\
40.6 \\
17.2 \\
32.2 \\
33.3 \\
30.0 \\
33.9 \\
24.4 \\
14.4 \\
22.8 \\
28.3 \\
21.1 \\
30.6 \\
22.2 \\
32.2 \\
36.1 \\
31.7\end{array}$ & $\begin{array}{rr}8 & 7.7 \\
9 & 8.2 \\
2 & 8.2 \\
3 & 7.9 \\
7 & 8.1 \\
9 & 7.6 \\
9 & 9.6 \\
3 & 9.6 \\
3 & 7.5 \\
6 & 9.9 \\
0 & 7.9 \\
8 & 9.9 \\
3 & 10.0 \\
6 & 8.3\end{array}$ & $\begin{array}{l}23 \\
24 \\
22 \\
24 \\
20 \\
27 \\
23 \\
31 \\
24 \\
28 \\
29 \\
24 \\
22 \\
24 \\
22 \\
21 \\
25 \\
23 \\
30\end{array}$ & . & \\
\hline
\end{tabular}


APPENDIX A (Cont)

\begin{tabular}{|c|c|c|c|c|c|c|c|c|c|c|c|c|c|}
\hline $\begin{array}{l}\text { WELL } \\
\text { NO }\end{array}$ & & & & $\begin{array}{l}\text { TITUDE } \\
\text { EGREES }\end{array}$ & $\begin{array}{l}\text { LONG ITUDE } \\
\text { DEGREES }\end{array}$ & $\underset{\mathbf{M}}{\text { ELEV }}$ & $\underset{M}{\text { KBELV }}$ & $\underset{M}{\text { DEPTH }}$ & $\underset{\mathrm{C}}{\mathrm{BHT}}$ & $\underset{\mathrm{C}}{\text { STEMP }}$ & $\begin{array}{l}\text { GRADIENT } \\
\text { /KM }\end{array}$ & $\underset{\mathrm{C}}{\mathrm{BHTCOR}}$ & $\begin{array}{l}\text { AAPGRAD } \\
\text { C/RM }\end{array}$ \\
\hline $\begin{array}{l}12105 \\
12106 \\
12130 \\
12131 \\
12132 \\
12133 \\
12134 \\
12135 \\
12148 \\
12149 \\
12151 \\
12152 \\
12163 \\
12167 \\
12168 \\
12169 \\
12171 \\
12173 \\
12174 \\
12175 \\
12178 \\
12179 \\
12186 \\
12188 \\
12189 \\
12190 \\
12191 \\
12198 \\
12202 \\
12203 \\
12204 \\
12210 \\
12211 \\
12213 \\
12214 \\
12215 \\
12216 \\
12219 \\
12220 \\
12232 \\
12233 \\
12234 \\
12235 \\
12236 \\
12237 \\
12238 \\
12239 \\
12240 \\
12242 \\
12243 \\
12244 \\
12246 \\
12258 \\
12259 \\
12262 \\
\end{array}$ & $\begin{array}{l}8 \\
7 \\
9 \\
9 \\
8 \\
8 \\
8 \\
8\end{array}$ & $\begin{array}{r}1 \\
9 \\
3 \\
23 \\
14 \\
90\end{array}$ & $\begin{array}{l}76 \\
76 \\
76 \\
76 \\
76 \\
76 \\
76 \\
76 \\
76 \\
76 \\
76 \\
76 \\
76 \\
76 \\
76 \\
76 \\
76 \\
76 \\
76 \\
76 \\
76 \\
76 \\
76 \\
76 \\
76 \\
76 \\
76 \\
76 \\
76 \\
76 \\
76 \\
76 \\
76 \\
76 \\
76 \\
77 \\
76 \\
76 \\
76 \\
76 \\
76 \\
76 \\
76 \\
76 \\
76 \\
76 \\
76 \\
76 \\
76 \\
76 \\
76 \\
76 \\
76 \\
76 \\
76\end{array}$ & $\begin{array}{l}42.4732 \\
42.4734 \\
42.3864 \\
42.3836 \\
42.3892 \\
42.3849 \\
42.4848 \\
42.9452 \\
42.7894 \\
42.5978 \\
42.0413 \\
42.4565 \\
42.9369 \\
42.4630 \\
42.4628 \\
42.4591 \\
42.4584 \\
42.4723 \\
42.4661 \\
42.4638 \\
42.7908 \\
42.2545 \\
42.5130 \\
42.5203 \\
42.5169 \\
42.0856 \\
42.4977 \\
42.4774 \\
42.4963 \\
42.0413 \\
42.2968 \\
42.4512 \\
42.4520 \\
42.4391 \\
42.4333 \\
42.4288 \\
42.4284 \\
42.5171 \\
42.4997 \\
42.3984 \\
42.3920 \\
42.4156 \\
42.4095 \\
42.4091 \\
42.4001 \\
42.4001 \\
42.3999 \\
42.4008 \\
42.3951 \\
42.3848 \\
42.4080 \\
42.4009 \\
42.4954 \\
42.5124 \\
42.4542\end{array}$ & $\begin{array}{l}79.1502 \\
79.1578 \\
79.4070 \\
79.4984 \\
79.4167 \\
79.4223 \\
79.3023 \\
78.3227 \\
76.1742 \\
76.2958 \\
79.4684 \\
79.3174 \\
76.3460 \\
79.3833 \\
79.3756 \\
79.3892 \\
79.3702 \\
79.3561 \\
79.3416 \\
79.3480 \\
78.1754 \\
77.5033 \\
79.0892 \\
79.0839 \\
79.0633 \\
79.6419 \\
79.1009 \\
79.3564 \\
79.1543 \\
79.4583 \\
79.3343 \\
79.4028 \\
79.3769 \\
79.4003 \\
79.3855 \\
79.3907 \\
79.3991 \\
79.0746 \\
79.0894 \\
79.2068 \\
79.2086 \\
79.2398 \\
79.2485 \\
79.2390 \\
79.2355 \\
79.2483 \\
79.2562 \\
79.2633 \\
79.2526 \\
79.2553 \\
79.2733 \\
79.2797 \\
79.0752 \\
79.0684 \\
79.3960\end{array}$ & $\begin{array}{l}338.9 \\
318.5 \\
294.1 \\
278.9 \\
247.5 \\
243.2 \\
606.6 \\
279.5 \\
556.9 \\
483.1 \\
495.3 \\
216.4 \\
303.9 \\
182.9 \\
185.9 \\
183.8 \\
190.8 \\
187.5 \\
194.8 \\
195.1 \\
457.8 \\
536.8 \\
263.7 \\
267.6 \\
268.5 \\
531.6 \\
281.9 \\
183.2 \\
248.4 \\
516.6 \\
460.6 \\
180.1 \\
185.9 \\
189.0 \\
202.7 \\
210.9 \\
202.1 \\
264.6 \\
266.7 \\
429.8 \\
461.8 \\
524.9 \\
494.4 \\
518.8 \\
527.3 \\
499.3 \\
479.1 \\
446.8 \\
481.6 \\
458.7 \\
408.1 \\
392.0 \\
279.8 \\
269.7 \\
181.7\end{array}$ & $\begin{array}{l}341.4 \\
320.6 \\
297.5 \\
282.5 \\
250.9 \\
246.3 \\
609.9 \\
279.5 \\
559.6 \\
486.8 \\
499.0 \\
217.6 \\
306.6 \\
185.9 \\
189.0 \\
185.9 \\
191.4 \\
190.5 \\
197.8 \\
198.01 \\
461.2 \\
540.4 \\
266.1 \\
270.7 \\
271.6 \\
534.9 \\
284.01 \\
185.6 \\
249.3 \\
520.3 \\
463.0 \\
181.7 \\
189.00 \\
192.0 \\
204.2 \\
214.0 \\
205.1 \\
266.7 \\
269.1 \\
432.2 \\
464.2 \\
527.3 \\
496.5 \\
520.3 \\
529.7 \\
501.4 \\
481.03 \\
449.00 \\
483.7 \\
460.9 \\
410.6 \\
394.4 \\
282.9 \\
272.8 \\
184.7 \\
\end{array}$ & $\begin{array}{r}816.3 \\
791.6 \\
807.7 \\
796.7 \\
768.1 \\
769.0 \\
623.6 \\
409.7 \\
1268.9 \\
1386.8 \\
1312.2 \\
669.6 \\
1274.4 \\
623.9 \\
641.6 \\
635.8 \\
640.4 \\
625.8 \\
643.1 \\
650.4 \\
1625.2 \\
1216.2 \\
725.7 \\
732.1 \\
723.3 \\
1290.2 \\
745.2 \\
630.6 \\
698.9 \\
1351.2 \\
1079.3 \\
628.2 \\
650.7 \\
652.0 \\
677.9 \\
690.1 \\
681.2 \\
719.3 \\
735.8 \\
9014.8 \\
1035.4 \\
1009.5 \\
1060.4 \\
1023.8 \\
1001.3 \\
970.8 \\
1000.4 \\
984.8 \\
920.8 \\
914.4 \\
764.7 \\
736.7 \\
627.9\end{array}$ & $\begin{array}{l}30.6 \\
28.9 \\
31.7 \\
36.1 \\
28.3 \\
28.3 \\
26.1 \\
15.0 \\
38.9 \\
41.1 \\
35.6 \\
26.1 \\
37.2 \\
26.7 \\
26.7 \\
26.7 \\
26.7 \\
27.7 \\
26.7 \\
27.8 \\
58.3 \\
37.8 \\
29.4 \\
30.0 \\
28.3 \\
31.1 \\
28.9 \\
26.7 \\
28.9 \\
36.7 \\
30.0 \\
27.2 \\
26.7 \\
25.6 \\
27.8 \\
26.7 \\
26.7 \\
28.9 \\
28.9 \\
31.1 \\
31.1 \\
34.4 \\
34.4 \\
30.6 \\
34.4 \\
33.3 \\
33.3 \\
27.5 \\
31.1 \\
31.1 \\
28.3 \\
32.8 \\
26.7 \\
27.8 \\
22.8 \\
\end{array}$ & $\begin{array}{rr}6 & 8.5 \\
9 & 8.7 \\
7 & 9.1 \\
1 & 9.3 \\
3 & 9.6 \\
3 & 9.6 \\
1 & 5.8 \\
0 & 8.0 \\
9 & 5.6 \\
1 & 6.7 \\
6 & 8.0 \\
1 & 9.7 \\
2 & 7.9 \\
7 & 10.0 \\
7 & 10.0 \\
7 & 10.0 \\
7 & 10.0 \\
2 & 10.0 \\
7 & 9.9 \\
8 & 9.9 \\
3 & 6.6 \\
8 & 6.9 \\
4 & 9.1 \\
0 & 9.1 \\
3 & 9.1 \\
1 & 7.5 \\
9 & 9.0 \\
7 & 10.0 \\
9 & 9.3 \\
7 & 7.8 \\
0 & 7.7 \\
2 & 10.1 \\
7 & 10.0 \\
6 & 10.0 \\
8 & 9.9 \\
7 & 9.8 \\
7 & 9.9 \\
9 & 9.1 \\
9 & 9.1 \\
1 & 7.8 \\
1 & 7.5 \\
4 & 6.8 \\
4 & 7.1 \\
6 & 6.9 \\
4 & 6.8 \\
3 & 7.1 \\
3 & 7.3 \\
2 & 7.6 \\
1 & 7.3 \\
1 & 7.5 \\
3 & 8.0 \\
8 & 8.1 \\
7 & 9.0 \\
8 & 9.1 \\
8 & 10.1\end{array}$ & $\begin{array}{l}27.0 \\
25.5 \\
27.9 \\
33.7 \\
24.4 \\
24.3 \\
32.5 \\
17.1 \\
26.2 \\
24.8 \\
21.0 \\
24.4 \\
23.0 \\
26.7 \\
26.0 \\
26.1 \\
26.0 \\
27.6 \\
26.1 \\
27.5 \\
31.8 \\
25.4 \\
28.0 \\
28.6 \\
26.6 \\
18.3 \\
26.7 \\
26.4 \\
28.0 \\
21.4 \\
20.6 \\
27.2 \\
25.6 \\
23.8 \\
26.3 \\
24.4 \\
24.6 \\
27.5 \\
26.8 \\
23.9 \\
23.3 \\
26.7 \\
27.1 \\
23.2 \\
26.1 \\
25.6 \\
26.0 \\
20.2 \\
23.8 \\
23.9 \\
22.1 \\
26.9 \\
23.1 \\
25.4 \\
20.2\end{array}$ & $\begin{array}{r}4.6 \\
4.5 \\
4.6 \\
4.5 \\
4.3 \\
4.3 \\
3.3 \\
1.8 \\
7.8 \\
8.6 \\
8.1 \\
3.6 \\
7.8 \\
3.3 \\
3.4 \\
3.4 \\
3.4 \\
3.3 \\
3.4 \\
3.5 \\
10.3 \\
7.4 \\
4.0 \\
4.1 \\
4.0 \\
7.9 \\
4.1 \\
3.3 \\
3.8 \\
8.4 \\
6.5 \\
3.3 \\
3.5 \\
3.5 \\
3.7 \\
3.8 \\
3.7 \\
4.0 \\
4.1 \\
5.7 \\
6.0 \\
6.2 \\
6.0 \\
6.1 \\
6.3 \\
6.1 \\
5.9 \\
5.7\end{array}$ & $\begin{array}{l}32.7 \\
31.2 \\
33.6 \\
39.3 \\
30.0 \\
29.9 \\
37.9 \\
21.4 \\
32.4 \\
31.1 \\
27.2 \\
29.8 \\
29.2 \\
31.9 \\
31.3 \\
31.5 \\
31.4 \\
32.9 \\
31.4 \\
32.8 \\
38.2 \\
31.5 \\
33.5 \\
34.1 \\
32.1 \\
24.4 \\
32.4\end{array}$ \\
\hline
\end{tabular}


APPENDIX A (Cont)

\begin{tabular}{|c|c|c|c|c|c|c|c|c|c|c|c|c|c|}
\hline $\begin{array}{l}\text { WELL } \\
\text { NO }\end{array}$ & & & & $\begin{array}{r}\text { LATITUDE } \\
\text { DEGREES }\end{array}$ & $\begin{array}{l}\text { LONG ITUDE } \\
\text { DEGREES }\end{array}$ & $\underset{M}{\text { ELEV }}$ & $\underset{M}{\text { KBELV }}$ & $\underset{M}{\text { DEPTH }}$ & $\underset{\text { C }}{\text { BHT }}$ & $\begin{array}{c}\text { STEMP } \\
\mathrm{C}\end{array}$ & $\begin{array}{l}\text { GRADI ENT } \\
\text { C/KM }\end{array}$ & $\underset{\text { C }}{\text { BHTCOR }}$ & $\begin{array}{l}\text { AAPGRAD } \\
\text { C/RM }\end{array}$ \\
\hline $\begin{array}{l}12264 \\
12265 \\
12266 \\
12267 \\
12273 \\
12274 \\
12275 \\
12276 \\
12277 \\
12278 \\
12286 \\
12287 \\
12288 \\
12289 \\
12290 \\
12292 \\
12294 \\
12296 \\
12297 \\
12298 \\
12299 \\
12300 \\
12301 \\
12302 \\
12305 \\
12306 \\
12307 \\
12309 \\
12310 \\
12329 \\
12332 \\
12339 \\
12350 \\
12351 \\
12359 \\
12366 \\
12367 \\
12383 \\
12385 \\
12386 \\
12387 \\
12388 \\
12390 \\
12391 \\
12393 \\
12397 \\
12397 \\
12398 \\
12399 \\
12399 \\
12402 \\
12403 \\
12406 \\
12408 \\
12411 \\
\end{array}$ & $\begin{array}{r}9 \\
9 \\
9 \\
9 \\
10 \\
9 \\
11 \\
9 \\
9 \\
12 \\
9 \\
11 \\
11 \\
10 \\
11 \\
10 \\
9 \\
9 \\
10 \\
10 \\
10 \\
9 \\
11 \\
11 \\
9 \\
10 \\
10 \\
10 \\
10 \\
9 \\
9 \\
10 \\
8 \\
11 \\
11 \\
11 \\
10 \\
11 \\
9 \\
10 \\
12 \\
11 \\
11 \\
1\end{array}$ & $\begin{array}{r}4 \\
9 \\
15 \\
22 \\
8 \\
29 \\
11 \\
21 \\
2 \\
16 \\
5 \\
21 \\
1 \\
18 \\
11 \\
24 \\
13 \\
30 \\
22 \\
6 \\
12 \\
27 \\
9 \\
1 \\
30 \\
7 \\
8 \\
1 \\
8 \\
20 \\
29 \\
16 \\
5 \\
23 \\
17 \\
3 \\
23 \\
12 \\
16 \\
21\end{array}$ & $\begin{array}{l}76 \\
76 \\
76 \\
76 \\
76 \\
76 \\
76 \\
76 \\
76 \\
76 \\
76 \\
76 \\
76 \\
76 \\
76 \\
76 \\
76 \\
76 \\
76 \\
76 \\
78 \\
76 \\
76 \\
76 \\
76 \\
76 \\
76 \\
76 \\
76 \\
76 \\
76 \\
76 \\
76 \\
77 \\
76 \\
76 \\
76 \\
76 \\
76 \\
76 \\
76 \\
76\end{array}$ & $\begin{array}{l}42.4912 \\
42.4739 \\
42.4679 \\
42.4650 \\
42.4233 \\
42.4199 \\
42.4143 \\
42.4093 \\
42.4065 \\
42.3988 \\
42.4064 \\
42.5929 \\
42.5921 \\
42.5884 \\
42.5870 \\
42.5929 \\
42.4067 \\
42.3994 \\
42.4347 \\
42.4295 \\
42.4339 \\
42.4301 \\
42.4202 \\
42.4186 \\
42.4879 \\
42.4819 \\
42.4774 \\
42.4754 \\
42.4800 \\
42.5000 \\
42.0478 \\
42.4162 \\
42.5910 \\
42.4139 \\
42.7160 \\
42.3943 \\
42.3893 \\
42.8273 \\
42.4032 \\
42.3954 \\
42.3970 \\
42.3918 \\
42.3902 \\
42.3861 \\
42.7715 \\
42.4217 \\
42.4616 \\
43.4868 \\
43.5087 \\
43.5087 \\
42.3167 \\
42.5354 \\
43.5019 \\
42.9613 \\
42.3902\end{array}$ & $\begin{array}{l}79.0829 \\
79.1256 \\
79.1284 \\
79.1398 \\
79.2089 \\
79.2155 \\
79.2121 \\
79.2084 \\
79.2037 \\
79.2151 \\
79.2831 \\
79.1061 \\
79.0975 \\
79.1142 \\
79.0542 \\
79.0653 \\
79.2176 \\
79.2886 \\
79.2333 \\
79.2280 \\
79.2186 \\
79.2385 \\
79.2686 \\
79.2775 \\
79.1491 \\
79.1470 \\
79.1415 \\
79.1333 \\
79.1279 \\
79.0687 \\
79.4994 \\
79.2845 \\
78.7154 \\
79.2555 \\
78.8788 \\
79.1830 \\
79.1848 \\
78.6394 \\
79.2417 \\
79.2299 \\
79.2420 \\
79.2369 \\
79.2441 \\
79.2394 \\
77.4962 \\
79.2859 \\
79.3384 \\
76.1890 \\
76.1958 \\
76.1958 \\
77.3822 \\
78.5111 \\
76.2002 \\
78.3170 \\
79.4244\end{array}$ & $\begin{array}{l}318.5 \\
369.4 \\
379.8 \\
377.3 \\
376.7 \\
410.0 \\
406.6 \\
396.2 \\
127.3 \\
457.2 \\
368.2 \\
189.6 \\
191.1 \\
181.7 \\
202.7 \\
197.5 \\
455.1 \\
362.7 \\
431.3 \\
445.3 \\
524.0 \\
473.0 \\
453.8 \\
367.3 \\
275.8 \\
313.3 \\
338.9 \\
353.6 \\
344.4 \\
292.3 \\
533.4 \\
332.4 \\
327.7 \\
488.3 \\
233.2 \\
121.2 \\
423.1 \\
248.4 \\
510.5 \\
533.4 \\
520.6 \\
543.2 \\
515.4 \\
505.4 \\
320.0 \\
329.5 \\
199.6 \\
119 . .2 \\
103.0 \\
103.0 \\
521.55 \\
424.3 \\
100.0 \\
281.6 \\
238.4\end{array}$ & $\begin{array}{l}320.6 \\
372.5 \\
382.8 \\
380.4 \\
376.7 \\
412.1 \\
408.7 \\
398.4 \\
427.4 \\
459.6 \\
370.6 \\
191.4 \\
192.9 \\
183.2 \\
204.5 \\
199.3 \\
457.4 \\
365.2 \\
433.7 \\
447.4 \\
526.4 \\
475.5 \\
456.3 \\
369.7 \\
278.9 \\
316.4 \\
342.0 \\
356.6 \\
346.3 \\
295.4 \\
536.4 \\
334.7 \\
330.7 \\
490.7 \\
234.7 \\
423.1 \\
425.1 \\
249.9 \\
512.7 \\
535.8 \\
523.0 \\
545.6 \\
517.9 \\
507.8 \\
322.8 \\
331.9 \\
202.7 \\
119.8 \\
103.6 \\
103.6 \\
525.8 \\
427.0 \\
100.6 \\
284.1 \\
240.8\end{array}$ & $\begin{array}{r}782.7 \\
872.0 \\
887.3 \\
872.0 \\
890.3 \\
926.9 \\
931.2 \\
932.4 \\
898.6 \\
989.7 \\
954.0 \\
556.0 \\
558.1 \\
554.4 \\
574.9 \\
586.7 \\
973.5 \\
892.5 \\
933.0 \\
939.4 \\
905.3 \\
961.6 \\
930.9 \\
882.4 \\
736.4 \\
785.2 \\
822.4 \\
841.9 \\
820.8 \\
773.3 \\
1358.2 \\
855.6 \\
763.5 \\
997.0 \\
506.0 \\
972.6 \\
981.8 \\
460.6 \\
1026.9 \\
1067.7 \\
1053.4 \\
1083.6 \\
1061.9 \\
1045.5 \\
347.2 \\
836.7 \\
656.8 \\
556.0 \\
544.1 \\
544.1 \\
1791.6 \\
980.5 \\
546.5 \\
396.2 \\
748.9\end{array}$ & $\begin{array}{l}30.6 \\
31.1 \\
32.2 \\
30.6 \\
30.0 \\
32.2 \\
27.8 \\
29.4 \\
32.2 \\
31.7 \\
33.9 \\
25.6 \\
26.7 \\
26.7 \\
24.4 \\
25.6 \\
31.1 \\
31.7 \\
27.8 \\
29.4 \\
28.9 \\
27.2 \\
28.9 \\
29.4 \\
30.0 \\
29.4 \\
30.6 \\
31.7 \\
28.3 \\
28.9 \\
37.8 \\
29.4 \\
26.1 \\
28.3 \\
18.3 \\
29.4 \\
30.0 \\
17.2 \\
29.4 \\
32.8 \\
26.7 \\
31.7 \\
32.2 \\
32.2 \\
17.8 \\
28.3 \\
27.8 \\
23.3 \\
18.3 \\
18.9 \\
47.2 \\
33.3 \\
22.8 \\
13.9 \\
28.3\end{array}$ & $\begin{array}{ll}6 & 8.7 \\
1 & 8.2 \\
2 & 8.1 \\
6 & 8.1 \\
0 & 8.3 \\
2 & 7.9 \\
8 & 8.0 \\
4 & 8.1 \\
2 & 7.8 \\
7 & 7.5 \\
9 & 8.4 \\
6 & 9.7 \\
7 & 9.7 \\
7 & 9.8 \\
4 & 9.6 \\
6 & 9.6 \\
1 & 7.5 \\
7 & 8.4 \\
8 & 7.7 \\
4 & 7.6 \\
9 & 6.8 \\
2 & 7.3 \\
9 & 7.5 \\
4 & 8.3 \\
0 & 9.1 \\
4 & 8.7 \\
6 & 8.5 \\
7 & 8.3 \\
3 & 8.4 \\
9 & 8.9 \\
8 & 7.6 \\
4 & 8.7 \\
1 & 8.3 \\
3 & 7.2 \\
3 & 9.0 \\
4 & 7.9 \\
0 & 7.9 \\
2 & 8.6\end{array}$ & $\begin{array}{l}28.0 \\
26.3 \\
27.2 \\
25.7 \\
24.4 \\
26.2 \\
21.3 \\
22.9 \\
27.2 \\
24.4 \\
26.8 \\
28.6 \\
30.5 \\
30.5 \\
25.9 \\
27.2 \\
24.2 \\
26.0 \\
21.5 \\
23.3 \\
24.4 \\
20.7 \\
23.0 \\
23.9 \\
28.4 \\
26.4 \\
26.8 \\
27.7 \\
24.2 \\
25.9 \\
22.2 \\
24.3 \\
23.3 \\
21.2 \\
18.5 \\
22.2 \\
22.5 \\
18.8 \\
21.9 \\
24.4 \\
18.8 \\
23.1 \\
23.8 \\
24.1 \\
28.1 \\
23.5 \\
27.3 \\
26.4 \\
17.6 \\
18.6 \\
22.5 \\
26.3 \\
25.5 \\
15.0 \\
24.9\end{array}$ & $\begin{array}{r}4.4 \\
5.0 \\
5.1 \\
5.0 \\
5.2 \\
5.4 \\
5.4 \\
5.4 \\
5.2 \\
5.9 \\
5.6 \\
2.8 \\
2.8 \\
2.8 \\
2.8 \\
3.0 \\
3.0 \\
5.7 \\
5.2 \\
5.5 \\
5.5 \\
5.3 \\
5.7 \\
5.4 \\
5.1 \\
4.1 \\
4.4 \\
4.7 \\
4.7 \\
4.7 \\
4.7 \\
4.3 \\
8.4 \\
4.9\end{array}$ & $\begin{array}{l}33.6 \\
32.1 \\
33.0 \\
31.5 \\
30.2 \\
32.1 \\
27.1 \\
28.8 \\
33.0 \\
30.3 \\
32.6 \\
33.6 \\
35.6 \\
35.6 \\
31.0 \\
32.4 \\
30.1 \\
31.8 \\
27.4 \\
29.2 \\
30.2 \\
26.6 \\
28.8 \\
29.7 \\
34.0 \\
32.0 \\
32.5 \\
33.4 \\
29.9 \\
31.5 \\
28.4 \\
30.0 \\
28.9 \\
27.1 \\
23.4 \\
28.1 \\
28.4 \\
23.5 \\
27.8 \\
30.3 \\
24.7 \\
29.1 \\
29.8 \\
30.0 \\
32.0 \\
29.2 \\
32.6 \\
31.4 \\
22.6 \\
23.6 \\
28.9 \\
32.2 \\
30.6 \\
19.3 \\
30.5\end{array}$ \\
\hline
\end{tabular}


APPENDIX A (Cont)

\begin{tabular}{|c|c|c|c|c|c|c|c|c|c|c|c|c|}
\hline $\begin{array}{l}\text { WELL } \\
\text { NO }\end{array}$ & & ITE & $\begin{array}{c}\text { LATITUDE } \\
\text { DEGREES }\end{array}$ & $\begin{array}{l}\text { LONG ITUDE } \\
\text { DEGREES }\end{array}$ & $\underset{M}{\text { ELEV }}$ & $\underset{M}{\text { RBELV }}$ & $\underset{\mathbf{M}}{\text { DEPTH }}$ & $\underset{\mathrm{C}}{\mathrm{BHT}} \mathrm{s}$ & $\underset{C}{\text { STEMP }}$ & $\begin{array}{l}\text { RADIENT } \\
C / \mathbb{R M}\end{array}$ & $\underset{\mathrm{C}}{\mathrm{HTCOR}}$ & $\begin{array}{l}\text { APGRAD } \\
\text { C/KM }\end{array}$ \\
\hline $\begin{array}{l}12411 \\
12413 \\
12414 \\
12418 \\
12419 \\
12421 \\
12423 \\
12426 \\
12427 \\
12445 \\
12447 \\
12450 \\
12452 \\
12458 \\
12459 \\
12461 \\
12465 \\
12480 \\
12481 \\
12482 \\
12505 \\
12550 \\
12553 \\
12564 \\
12578 \\
12579 \\
12580 \\
12581 \\
12582 \\
12583 \\
12584 \\
12585 \\
12587 \\
12588 \\
12589 \\
12591 \\
12592 \\
12593 \\
12594 \\
12597 \\
12598 \\
12599 \\
12600 \\
12601 \\
12602 \\
12603 \\
12604 \\
12605 \\
12606 \\
12607 \\
12609 \\
12610 \\
12611 \\
12612 \\
12614\end{array}$ & 1 & 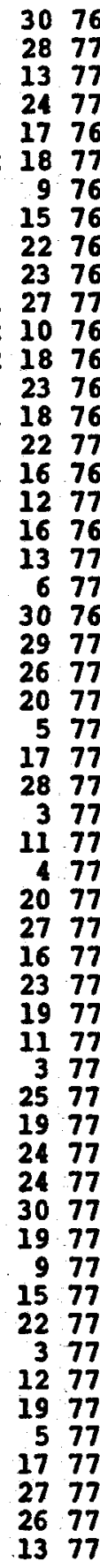 & $\begin{array}{l}42.3902 \\
42.3770 \\
42.3713 \\
42.4628 \\
42.4255 \\
42.3819 \\
42.4024 \\
42.4325 \\
42.4251 \\
42.3902 \\
42.5189 \\
42.7405 \\
42.7406 \\
42.2893 \\
42.4564 \\
42.4444 \\
42.5740 \\
42.4507 \\
42.2889 \\
42.2748 \\
42.4511 \\
42.4007 \\
42.4336 \\
42.8445 \\
42.0548 \\
42.0497 \\
42.0548 \\
42.0541 \\
42.0424 \\
42.0449 \\
42.0580 \\
42.0530 \\
42.0594 \\
42.0474 \\
42.0338 \\
42.0350 \\
42.0408 \\
42.0464 \\
42.0345 \\
42.2755 \\
42.2788 \\
42.2630 \\
42.2528 \\
42.2508 \\
42.2591 \\
42.2531 \\
42.2585 \\
42.2661 \\
42.2649 \\
42.2627 \\
42.2701 \\
42.2673 \\
42.2634 \\
42.2579 \\
42.2517\end{array}$ & $\begin{array}{l}79.4244 \\
79.3672 \\
79.3635 \\
79.1688 \\
79.1184 \\
79.2348 \\
79.2244 \\
79.2859 \\
79.2177 \\
79.2598 \\
76.1909 \\
78.5184 \\
78.5044 \\
79.4138 \\
78.8102 \\
78.8191 \\
79.0523 \\
78.8087 \\
79.4040 \\
79.3541 \\
78.8172 \\
79.2888 \\
79.1009 \\
78.4949 \\
79.4893 \\
79.4919 \\
79.4893 \\
79.4961 \\
79.4633 \\
79.5114 \\
79.5058 \\
79.4657 \\
79.4997 \\
79.4624 \\
79.4479 \\
79.1678 \\
79.1480 \\
79.4527 \\
79.4597 \\
79.5457 \\
79.4401 \\
79.4667 \\
79.4460 \\
79.4377 \\
79.4274 \\
79.4276 \\
79.4189 \\
79.4192 \\
79.4108 \\
79.4038 \\
79.3601 \\
79.3993 \\
79.3936 \\
79.3945 \\
79.3933\end{array}$ & $\begin{array}{l}238.4 \\
469.4 \\
466.3 \\
323.1 \\
189.0 \\
492.3 \\
484.9 \\
306.9 \\
402.0 \\
464.5 \\
94.8 \\
356.6 \\
326.1 \\
478.8 \\
289.0 \\
347.5 \\
198.1 \\
289.6 \\
508.4 \\
395.3 \\
289.6 \\
360.0 \\
193.5 \\
307.8 \\
533.1 \\
515.1 \\
524.3 \\
515.1 \\
522.7 \\
530.4 \\
550.2 \\
527.9 \\
527.3 \\
480.1 \\
531.9 \\
490.7 \\
518.2 \\
508.4 \\
521.2 \\
388.0 \\
499.9 \\
417.0 \\
438.9 \\
409.0 \\
424.3 \\
432.5 \\
451.1 \\
453.8 \\
465.7 \\
499.0 \\
523.3 \\
500.8 \\
522.4 \\
516.6 \\
521.2 \\
\\
\end{array}$ & 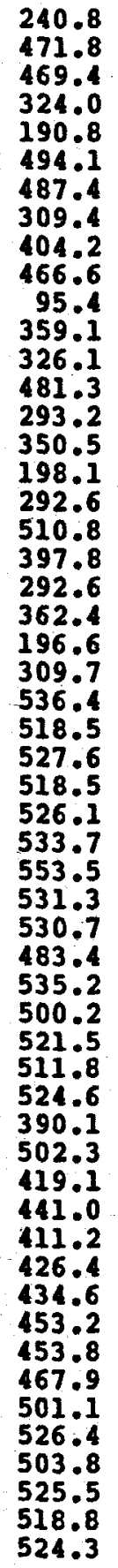 & $\begin{array}{r}748.9 \\
995.2 \\
1012.9 \\
792.5 \\
553.2 \\
1027.8 \\
1020.8 \\
803.8 \\
964.1 \\
996.1 \\
511.1 \\
678.2 \\
659.9 \\
1069.8 \\
876.3 \\
971.1 \\
585.5 \\
871.4 \\
1115.3 \\
1024.4 \\
864.1 \\
892.5 \\
665.1 \\
534.6 \\
1359.7 \\
1342.6 \\
1347.8 \\
1340.5 \\
1350.0 \\
1358.5 \\
1360.0 \\
1342.6 \\
1341.1 \\
1318.6 \\
1387.1 \\
1352.4 \\
1331.4 \\
1335.9 \\
1399.6 \\
1012.9 \\
1112.5 \\
1039.7 \\
1092.4 \\
1054.6 \\
1066.8 \\
1078.1 \\
1097.6 \\
1079.0 \\
1098.2 \\
1137.2 \\
1150.0 \\
1135.7 \\
1147.3\end{array}$ & $\begin{array}{l}27.8 \\
33.3 \\
30.6 \\
23.9 \\
25.6 \\
32.8 \\
30.6 \\
26.7 \\
30.6 \\
31.7 \\
18.9 \\
25.6 \\
26.7 \\
29.4 \\
30.6 \\
31.7 \\
21.1 \\
30.0 \\
26.7 \\
32.8 \\
30.0 \\
31.7 \\
22.2 \\
22.8 \\
37.2 \\
36.1 \\
36.7 \\
36.1 \\
38.9 \\
38.3 \\
33.3 \\
36.7 \\
36.1 \\
36.1 \\
37.8 \\
38.9 \\
40.0 \\
43.3 \\
38.3 \\
31.7 \\
32.8 \\
31.7 \\
33.3 \\
32.8 \\
31.1 \\
33.3 \\
33.3 \\
32.2 \\
33.3 \\
35.6 \\
33.3 \\
36.7 \\
37.2 \\
33.9 \\
30.0\end{array}$ & $\begin{array}{r}9.7 \\
7.4 \\
7.5 \\
8.7 \\
10.1 \\
7.2 \\
7.2 \\
8.9 \\
8.0 \\
7.5 \\
10.6 \\
7.7 \\
8.0 \\
7.6 \\
9.0 \\
8.5 \\
9.7 \\
9.0 \\
7.3 \\
8.4 \\
9.0 \\
8.5 \\
10.0 \\
7.9 \\
7.6 \\
7.8 \\
7.7 \\
7.7 \\
7.7 \\
7.6 \\
7.4 \\
7.6 \\
7.6 \\
8.1 \\
7.6 \\
8.0 \\
7.7 \\
7.8 \\
7.7 \\
8.5 \\
7.4 \\
8.2 \\
8.0 \\
8.3 \\
8.2 \\
8.1 \\
7.9 \\
7.9 \\
7.8 \\
7.4 \\
7.2 \\
7.4 \\
7.2\end{array}$ & $\begin{array}{l}24.2 \\
26.0 \\
22.8 \\
19.2 \\
28.0 \\
24.9 \\
22.9 \\
22.1 \\
23.4 \\
24.3 \\
16.2 \\
26.3 \\
28.2 \\
20.5 \\
24.6 \\
23.9 \\
19.6 \\
24.1 \\
17.4 \\
23.8 \\
24.3 \\
26.0 \\
18.4 \\
27.7 \\
21.8 \\
21.1 \\
21.5 \\
21.2 \\
23.1 \\
22.6 \\
19.1 \\
21.6 \\
21.2 \\
21.2 \\
21.7 \\
22.9 \\
24.2 \\
26.6 \\
21.9 \\
22.9 \\
22.8 \\
22.5 \\
23.1 \\
23.2 \\
21.5 \\
23.4 \\
23.2 \\
22.6 \\
23.3 \\
24.7 \\
22.8 \\
25.8 \\
26.2 \\
22.9 \\
19.5 \\
\end{array}$ & $\begin{array}{l}6 \\
4 \\
5 \\
5 \\
2 \\
3 \\
3 \\
6 \\
5\end{array}$ & $\begin{array}{l}29.1 \\
30.4 \\
32.8 \\
28.1 \\
28.8 \\
28.9 \\
28.9 \\
29.5 \\
29.1 \\
27.5 \\
29.5 \\
29.1 \\
28.6 \\
29.3 \\
30.8 \\
28.8 \\
31.8 \\
32.2 \\
28.9 \\
25.6\end{array}$ \\
\hline
\end{tabular}


APPENDIX A (Cont)

\begin{tabular}{|c|c|c|c|c|c|c|c|c|c|c|c|c|c|}
\hline $\begin{array}{c}\text { WELL } \\
\text { NO }\end{array}$ & & & & $\begin{array}{c}\text { LATITUDE } \\
\text { DEGREES }\end{array}$ & $\begin{array}{l}\text { LONG ITUDE } \\
\text { DEGREES }\end{array}$ & $\begin{array}{c}\text { ELEV } \\
M\end{array}$ & $\underset{M}{\text { KBELV }}$ & $\underset{M}{\text { DEPTH }}$ & $\underset{\mathrm{C}}{\mathrm{BHT}}$ & $\begin{array}{l}\text { PEMP } \\
\text { C }\end{array}$ & $\begin{array}{l}\text { GRADIENT } \\
\mathrm{C} / \mathrm{KM}\end{array}$ & $\underset{\text { C }}{\text { BHTCOR }}$ & $\begin{array}{c}\text { AAPGRA } \\
\text { C/KM }\end{array}$ \\
\hline $\begin{array}{l}12615 \\
12616 \\
12623 \\
12624 \\
12625 \\
12641 \\
12654 \\
12676 \\
12677 \\
12703 \\
12706 \\
12719 \\
12721 \\
12724 \\
12731 \\
12745 \\
12745 \\
12745 \\
12765 \\
12778 \\
12782 \\
12783 \\
12784 \\
12800 \\
12805 \\
12806 \\
12812 \\
12813 \\
12819 \\
12837 \\
12848 \\
12859 \\
12891 \\
12892 \\
12893 \\
12894 \\
12895 \\
12910 \\
12927 \\
12944 \\
12960 \\
12970 \\
12972 \\
12973 \\
12974 \\
12975 \\
12982 \\
12983 \\
12985 \\
13011 \\
13043 \\
13050 \\
13058 \\
13082 \\
13083 \\
\end{array}$ & $\begin{array}{r}5 \\
7 \\
1 \\
1 \\
12 \\
3 \\
2 \\
3 \\
3 \\
6 \\
11 \\
4 \\
5 \\
5 \\
5 \\
5 \\
6 \\
6 \\
6 \\
7 \\
7 \\
7 \\
7 \\
9 \\
7 \\
8 \\
8 \\
8 \\
6 \\
7 \\
10 \\
11 \\
9 \\
9\end{array}$ & $\begin{array}{r}29 \\
7 \\
5 \\
17 \\
22 \\
22 \\
25 \\
9 \\
17 \\
29 \\
2 \\
14 \\
16 \\
6 \\
5 \\
23 \\
7 \\
4 \\
18 \\
14 \\
15 \\
20 \\
21 \\
10 \\
7 \\
25 \\
16 \\
55 \\
30 \\
27 \\
10 \\
14 \\
27 \\
30 \\
30 \\
30\end{array}$ & $\begin{array}{l}77 \\
77 \\
77 \\
77 \\
77 \\
78 \\
77 \\
77 \\
77 \\
77 \\
77 \\
78 \\
77 \\
77\end{array}$ & $\begin{array}{l}42.2524 \\
42.2512 \\
42.3955 \\
42.3707 \\
42.3679 \\
42.8348 \\
42.4945 \\
42.3434 \\
42.3498 \\
42.1233 \\
42.7865 \\
42.8458 \\
42.8421 \\
42.8432 \\
42.4179 \\
42.5792 \\
42.5792 \\
42.5792 \\
42.1980 \\
42.8451 \\
42.8528 \\
42.8591 \\
42.8432 \\
42.9639 \\
42.0696 \\
42.5718 \\
42.5839 \\
42.5884 \\
42.8822 \\
42.4701 \\
42.8959 \\
42.4088 \\
42.9468 \\
42.9391 \\
42.9306 \\
42.9390 \\
42.9548 \\
42.4665 \\
42.1367 \\
42.0160 \\
42.3711 \\
42.5282 \\
42.2341 \\
42.0578 \\
42.0559 \\
42.0622 \\
42.0472 \\
42.5375 \\
42.5327 \\
42.0266 \\
42.0577 \\
42.4201 \\
42.4750 \\
42.7059 \\
42.6124\end{array}$ & $\begin{array}{l}79.4151 \\
79.3860 \\
79.2136 \\
79.2561 \\
79.2634 \\
78.7207 \\
79.3047 \\
79.5523 \\
79.5527 \\
79.6791 \\
77.8689 \\
78.6708 \\
78.1718 \\
78.1690 \\
79.3236 \\
78.7205 \\
78.7205 \\
78.7205 \\
77.6657 \\
78.6841 \\
78.6618 \\
78.6714 \\
78.6620 \\
78.6158 \\
75.5967 \\
78.9311 \\
76.9427 \\
76.9545 \\
78.6392 \\
79.3223 \\
78.3905 \\
76.8981 \\
78.3152 \\
78.3163 \\
78.3337 \\
78.3358 \\
78.3174 \\
78.8038 \\
77.9594 \\
79.3913 \\
77.3896 \\
78.7291 \\
77.3690 \\
79.4747 \\
79.4464 \\
79.4545 \\
79.4739 \\
78.7199 \\
78.71477 \\
77.5871 \\
79.4626 \\
79.3491 \\
79.3248 \\
78.3672 \\
78.4348\end{array}$ & $\begin{array}{l}473.7 \\
531.3 \\
466.3 \\
451.1 \\
475.5 \\
217.9 \\
189.3 \\
212.8 \\
205.4 \\
454.4 \\
177.4 \\
225.6 \\
342.3 \\
350.8 \\
273.4 \\
441.4 \\
441.4 \\
441.4 \\
668.1 \\
225.6 \\
230.1 \\
211.8 \\
227.1 \\
225.9 \\
570.0 \\
347.5 \\
223.4 \\
241.7 \\
178.0 \\
205.7 \\
285.6 \\
248.4 \\
280.1 \\
284.4 \\
279.8 \\
288.3 \\
276.8 \\
350.5 \\
467.9 \\
498.3 \\
404.2 \\
426.7 \\
586.7 \\
456.6 \\
499.9 \\
462.1 \\
464.8 \\
467.9 \\
454.2 \\
553.2 \\
454.2 \\
243.2 \\
198.1 \\
490.7 \\
461.8\end{array}$ & $\begin{array}{l}474.3 \\
533.4 \\
468.8 \\
453.8 \\
478.5 \\
220.1 \\
192.3 \\
215.8 \\
208.5 \\
457.2 \\
177.4 \\
227.1 \\
342.3 \\
350.8 \\
276.8 \\
444.4 \\
444.4 \\
444.4 \\
670.6 \\
227.1 \\
231.6 \\
213.4 \\
228.6 \\
228.6 \\
573.0 \\
349.0 \\
225.9 \\
244.1 \\
180.1 \\
205.7 \\
285.6 \\
248.4 \\
280.7 \\
286.8 \\
281.6 \\
288.3 \\
276.8 \\
353.6 \\
467.9 \\
502.3 \\
407.8 \\
429.8 \\
590.1 \\
460.6 \\
499.9 \\
466.0 \\
467.0 \\
470.9 \\
457.2 \\
557.2 \\
458.1 \\
245.1 \\
201.5 \\
493.8 \\
464.8\end{array}$ & $\begin{array}{r}1121.4 \\
1182.9 \\
1029.3 \\
1001.0 \\
1024.7 \\
394.7 \\
615.1 \\
740.4 \\
731.8 \\
1226.8 \\
199.9 \\
421.5 \\
422.5 \\
424.3 \\
773.3 \\
773.3 \\
1679.8 \\
1403.9 \\
1417.6 \\
424.9 \\
424.3 \\
394.1 \\
424.6 \\
340.8 \\
1349.0 \\
767.2 \\
419.7 \\
437.1 \\
338.3 \\
651.7 \\
483.1 \\
896.7 \\
408.7 \\
417.6 \\
417.6 \\
417.6 \\
394.1 \\
1777.6 \\
549.6 \\
1391.4 \\
911.0 \\
909.5 \\
1271.6 \\
1283.8 \\
1308.8 \\
1289.6 \\
1312.5 \\
967.1 \\
953.7 \\
1516.7 \\
1274.1 \\
736.1 \\
850.3 \\
950.7\end{array}$ & $\begin{array}{l}36.1 \\
33.9 \\
31.1 \\
30.6 \\
31.1 \\
23.3 \\
26.1 \\
27.2 \\
26.1 \\
36.1 \\
18.9 \\
16.1 \\
23.9 \\
19.4 \\
32.2 \\
28.3 \\
43.9 \\
41.7 \\
37.8 \\
17.8 \\
17.8 \\
16.7 \\
18.3 \\
15.6 \\
38.3 \\
23.3 \\
22.2 \\
20.6 \\
17.8 \\
27.2 \\
18.9 \\
27.8 \\
15.0 \\
15.0 \\
16.7 \\
13.9 \\
16.7 \\
48.9 \\
21.1 \\
38.3 \\
27.8 \\
30.0 \\
35.6 \\
36.7 \\
38.3 \\
34.4\end{array}$ & $\begin{array}{l}7.7 \\
7.1 \\
7.4 \\
7.6 \\
7.4 \\
8.8 \\
9.9 \\
10.0 \\
10.1 \\
8.2 \\
9.4 \\
8.7 \\
7.7 \\
7.6 \\
9.3 \\
7.2 \\
7.2 \\
7.2 \\
5.7 \\
8.7 \\
8.7 \\
8.8 \\
8.7 \\
8.4 \\
6.3 \\
8.2 \\
9.3 \\
9.1 \\
9.1 \\
9.8 \\
8.1 \\
9.4 \\
8.0 \\
8.0 \\
8.0 \\
7.9 \\
8.0 \\
8.4 \\
7.9 \\
8.0 \\
7.9 \\
7.5 \\
6.4 \\
8.4\end{array}$ & $\begin{array}{l}25.3 \\
22.6 \\
23.0 \\
22.9 \\
23.1 \\
36.8 \\
26.4 \\
23.2 \\
21.9 \\
22.7 \\
47.4 \\
17.5 \\
38.4 \\
28.0 \\
29.7 \\
27.3 \\
21.8 \\
24.5 \\
22.6 \\
21.3 \\
21.5 \\
19.9 \\
22.6 \\
20.9 \\
23.8 \\
19.7 \\
30.8 \\
26.1 \\
25.6 \\
26.7 \\
22.4 \\
20.5 \\
17.1 \\
16.9 \\
20.7 \\
14.2 \\
21.9 \\
22.8 \\
24.1 \\
21.8 \\
21.8 \\
24.7 \\
22.9 \\
22.1 \\
23.2 \\
20.3 \\
22.5 \\
21.4 \\
24.5 \\
23.5 \\
20.9 \\
27.8 \\
25.1 \\
20.5 \\
21.9\end{array}$ & $\begin{array}{r}6.8 \\
7.2 \\
6.1 \\
5.9 \\
6.1 \\
1.7 \\
3.2 \\
4.1 \\
4.0 \\
7.5 \\
.3 \\
1.9 \\
1.9 \\
1.9 \\
4.3 \\
4.3 \\
10.7 \\
8.7 \\
8.8 \\
1.9 \\
1.9 \\
1.7 \\
1.9 \\
1.3 \\
8.4 \\
4.3 \\
1.9 \\
2.0 \\
1.3 \\
3.5 \\
2.3 \\
5.2 \\
1.8 \\
1.9 \\
1.9 \\
1.9 \\
1.7 \\
11.4 \\
2.8 \\
8.7 \\
5.3 \\
5.3 \\
7.8\end{array}$ & $\begin{array}{l}31.4 \\
28.7 \\
29.0 \\
28.8 \\
29.1 \\
41.1 \\
31.6 \\
28.8 \\
27.4 \\
28.9 \\
49.0 \\
22.0 \\
42.9 \\
32.5 \\
35.3 \\
32.9 \\
28.2 \\
30.8 \\
28.9 \\
25.8 \\
25.9 \\
24.2 \\
27.1 \\
24.8 \\
30.0 \\
25.3 \\
35.2 \\
30.7 \\
29.5 \\
32.0 \\
27.2 \\
26.3\end{array}$ \\
\hline
\end{tabular}


APPENDIX A (Cont)

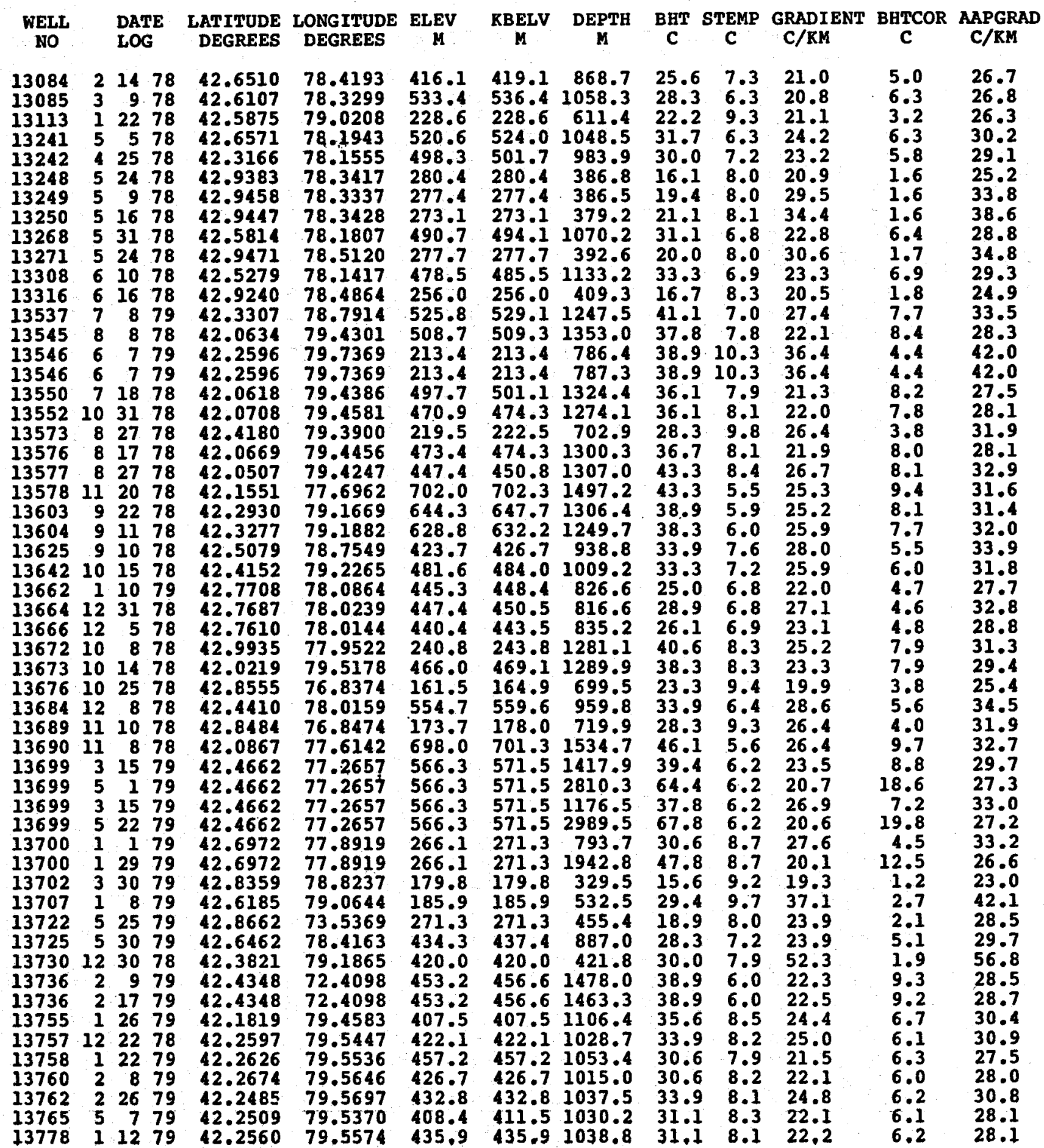


APPENDIX A (Cont)

\begin{tabular}{|c|c|c|c|c|c|c|c|c|c|c|c|}
\hline $\begin{array}{l}\text { WELL } \\
\text { NO }\end{array}$ & $\begin{array}{l}\text { DATE } \\
\text { LOG }\end{array}$ & $\begin{array}{r}\text { LATITUDE } \\
\text { DEGREES }\end{array}$ & $\begin{array}{c}\text { LONGIT } \\
\text { DEGRE }\end{array}$ & $\underset{\mathbf{M}}{\text { ELEV }}$ & BELV & $\underset{M}{\text { DEPTH }}$ & $\underset{\mathrm{C}}{\mathrm{BHT}}$ & $\underset{\mathrm{C}}{\text { STEMP }}$ & $\begin{array}{c}\text { GRADIENT } \\
\mathrm{C} / \mathrm{KM}\end{array}$ & $\underset{\mathrm{C}}{\mathrm{BHTCOR}}$ & $\begin{array}{c}\text { AAPGRAD } \\
\text { C/KM }\end{array}$ \\
\hline $\begin{array}{l}13782 \\
13790 \\
13796 \\
13812 \\
13841 \\
13854 \\
13859 \\
13862 \\
13887 \\
13891 \\
13892 \\
13893 \\
13907 \\
13919 \\
13982 \\
13983 \\
14178 \\
14250\end{array}$ & $\begin{array}{rrr}5 & 26 & 7 \\
3 & 15 & 7 \\
2 & 20 & 7 \\
1 & 13 & 7 \\
3 & 1 & 7 \\
2 & 2 & 7 \\
5 & 23 & 7 \\
2 & 10 & 7 \\
3 & 12 & 7 \\
3 & 16 & 7 \\
3 & 11 & 7 \\
3 & 1 & 7 \\
3 & 23 & 7 \\
4 & 21 & 7 \\
6 & 6 & 7 \\
5 & 24 & 7 \\
5 & 24 & 7 \\
6 & 14 & 7\end{array}$ & $\begin{array}{l}42.2468 \\
42.5988 \\
42.3888 \\
42.7983 \\
42.7350 \\
42.1905 \\
42.8288 \\
42.1858 \\
42.2147 \\
42.7797 \\
42.7587 \\
42.1844 \\
42.3080 \\
42.7587 \\
42.2720 \\
42.2746 \\
42.7239 \\
42.2786\end{array}$ & $\begin{array}{l}79.5471 \\
78.3792 \\
76.9328 \\
78.6156 \\
77.9534 \\
79.4558 \\
78.6629 \\
79.4546 \\
79.4818 \\
78.0483 \\
78.0845 \\
79.4639 \\
79.5961 \\
78.0777 \\
79.7075 \\
79.7124 \\
77.9726 \\
79.7057\end{array}$ & $\begin{array}{l}451.1 \\
512.4 \\
422.8 \\
275.8 \\
326.1 \\
403.9 \\
237.7 \\
402.9 \\
413.0 \\
463.3 \\
451.7 \\
408.4 \\
230.1 \\
455.7 \\
214.9 \\
207.3 \\
329.2 \\
207.0\end{array}$ & $\begin{array}{l}451.1 \\
515.4 \\
426.1 \\
275.8 \\
326.1 \\
403.9 \\
239.3 \\
402.9 \\
413.0 \\
463.3 \\
451.7 \\
408.4 \\
230.1 \\
456.3 \\
214.9 \\
208.2 \\
332.5 \\
207.0\end{array}$ & $\begin{array}{r}1073.2 \\
1043.0 \\
740.7 \\
525.8 \\
804.7 \\
1098.2 \\
448.1 \\
1101.2 \\
1085.7 \\
818.7 \\
822.7 \\
1099.7 \\
784.6 \\
830.9 \\
715.4 \\
763.2 \\
788.8 \\
761.7\end{array}$ & $\begin{array}{l}32.2 \\
29.4 \\
24.4 \\
31.1 \\
29.4 \\
35.6 \\
18.3 \\
35.0 \\
35.0 \\
26.7 \\
28.3 \\
38.9 \\
35.6 \\
25.0 \\
38.9 \\
32.2 \\
26.1 \\
28.3\end{array}$ & $\begin{array}{r}8.0 \\
6.5 \\
7.7 \\
8.4 \\
8.1 \\
8.6 \\
8.7 \\
8.6 \\
8.4 \\
6.6 \\
6.8 \\
8.5 \\
10.0 \\
6.7\end{array}$ & $\begin{array}{l}22.6 \\
22.0 \\
22.6 \\
43.2 \\
26.6 \\
24.6 \\
21.6 \\
24.0 \\
24.5 \\
24.5 \\
26.5 \\
27.6 \\
32.6 \\
22.0 \\
40.1 \\
28.7 \\
22.9 \\
23.7\end{array}$ & $\begin{array}{l}1.7 \\
3.9 \\
4.3 \\
4.4 \\
4.3\end{array}$ & $\begin{array}{l}48.2 \\
32.2 \\
30.6 \\
26.2 \\
30.2 \\
30.5 \\
30.5 \\
31.2 \\
33.6 \\
38.2 \\
27.7 \\
45.6 \\
34.3 \\
28.6 \\
29.3\end{array}$ \\
\hline
\end{tabular}




\section{APPENDIX B}

TEMPERATURE-DEPTH MEASUREMENTS AND TEMPERATURE-DEPTH PROFILES FOR WELLS MEASURED BY PERSONNEL OF THE STATE UNIVERSITY OF NEW YORK AT BUFFALO

TABLE B-I

WELL NO. 13738

PATTERSON-SPRINGPORT CAYUGA CO. NEW YORK 9-11-79

LATITUDE 14620. FEET $N$ of 42.50 .0$.

LONGITUDE 15100. FEET $W$ of 76. 37. 30.

ELEVATION 510. FEET

DEPIH TEPP

$M$ C

0.018 .01

$61.0 \quad 4.46$

$121.9 \quad 11.62$

$182.9 \quad 14.05$

$243.8 \quad 16.09$

$304.8 \quad 18.48$

$329.2 \quad 19.16$

$353.6 \quad 20.23$

$378.0 \quad 21.12$

$402.3 \quad 22.07$

$426.7 \quad 23.21$

451.124 .44

$475.5 \quad 25.55$

499.926 .04

$524.3 \quad 26.53$

$548.6 \quad 26.98$

$573.0 \quad 27.69$
DEPTH TERP

II C

$15.2 \quad 8.05$

76.26 .58

$\begin{array}{lll}137.2 & 12.47\end{array}$

$198.1 \quad 14.61$

$259.1 \quad 16.85$

310.918 .57

$\begin{array}{lll}335.3 & 19.38\end{array}$

$\begin{array}{ll}359.7 & 20.47\end{array}$

$384.0 \quad 21.32$

$408.4 \quad 22.55$

$432.8 \quad 23.53$

- 457.224 .85

$481.6 \quad 25.72$

$506.0 \quad 26.39$

$530.4 \quad 26.61$

554.727 .10
DEPIH TERP

$30.5 \quad 4.35$

$91.4 \quad 10.07$

$152.4 \quad 12.93$

$213.4 \quad 15.28$

$274.3 \quad 17.51$

$317.0 \quad 18.92$

$341.4 \quad 19.78$

$365.8 \quad 20.87$

$390.1 \quad 21.57$

$414.5 \quad 22.59$

$438.9 \quad 23.80$

$463.3 \quad 25.22$

487.725 .85

512.126 .74

$536.4 \quad 26.73$

$560.8 \quad 27.18$
DEPIH TE.PP

$\begin{array}{rr}45.7 & 4.37 \\ 106.7 & 10.15 \\ 167.6 & 13.52 \\ 228.6 & 15.53 \\ 289.6 & 17.94 \\ 323.1 & 18.98 \\ 347.5 & 19.95 \\ 371.9 & 20.97 \\ 396.2 & 21.80 \\ 420.6 & 22.90 \\ 445.0 & 24.07 \\ 469.4 & 25.41 \\ 493.8 & 26.01 \\ 518.2 & 26.70 \\ 542.5 & 27.09 \\ 566.9 & 27.41\end{array}$


APPENDIX B (Cont)

TABLE B-II

WELL NO. 13000 BLAKELY - N COLLINS ERIE C0., NEW YORK 7-7-79

LATITUDE 13200. FEET $S$ of 42.40 .0$.

LONGITUDE 10750. FEET W Of 78. 52. 30.

ELEVATION 856. FEET

\begin{tabular}{|c|c|c|c|c|c|c|}
\hline $\begin{array}{cc}\text { DEPTH } & \text { TEIP } \\
\mathbf{M} & \mathbf{C}\end{array}$ & $\underset{N}{\text { DEPIH }}$ & TERP & $\underset{11}{\text { DEPIH }}$ & $\underset{\mathrm{C}}{\mathrm{TEP}}$ & $\underset{\mathfrak{M}}{\mathrm{DEPTH}}$ & TENP \\
\hline $\begin{array}{rr}6.1 & 10.40 \\
18.3 & 10.54 \\
30.5 & 10.64 \\
42.7 & 10.79 \\
54.9 & 11.00 \\
67.1 & 11.27 \\
79.2 & 11.59 \\
91.4 & 12.03 \\
103.6 & 12.48 \\
115.8 & 12.92 \\
128.0 & 13.35 \\
140.2 & 13.61 \\
152.4 & 14.07 \\
164.6 & 14.53 \\
176.8 & 15.00 \\
189.0 & 15.45 \\
201.2 & 15.91 \\
213.4 & 16.38 \\
225.6 & 16.85 \\
237.7 & 17.32 \\
249.9 & 17.74 \\
262.1 & 18.15 \\
274.3 & 18.71 \\
286.5 & 18.96 \\
298.7 & 19.18 \\
310.9 & 19.40 \\
323.1 & 19.61 \\
335.3 & 19.80 \\
347.5 & 20.02 \\
359.7 & 20.19 \\
371.9 & 20.33 \\
384.0 & 20.48 \\
395.2 & 20.65 \\
408.4 & 20.91 \\
420.6 & 21.15 \\
432.8 & 21.38 \\
445.0 & 21.61\end{array}$ & $\begin{array}{r}9.1 \\
21.3 \\
33.5 \\
45.7 \\
57.9 \\
70.1 \\
82.3 \\
94.5 \\
106.7 \\
118.9 \\
131.1 \\
143.3 \\
155.4 \\
167.6 \\
179.8 \\
192.0 \\
204.2 \\
216.4 \\
228.6 \\
240.8 \\
253.0 \\
265.2 \\
277.4 \\
289.6 \\
301.8 \\
313.9 \\
326.1 \\
338.3 \\
350.5 \\
362.7 \\
374.9 \\
387.1 \\
399.3 \\
411.5 \\
423.7 \\
435.9 \\
448.1\end{array}$ & $\begin{array}{l}10.44 \\
10.56 \\
10.68 \\
10.84 \\
11.06 \\
11.34 \\
11.69 \\
12.15 \\
12.58 \\
13.02 \\
13.45 \\
13.71 \\
14.18 \\
14.66 \\
15.10 \\
15.56 \\
16.03 \\
16.49 \\
16.97 \\
17.43 \\
17.85 \\
18.29 \\
18.79 \\
19.01 \\
19.23 \\
19.46 \\
19.66 \\
19.85 \\
20.06 \\
20.23 \\
20.38 \\
20.52 \\
20.70 \\
20.98 \\
21.20 \\
21.42 \\
21.68\end{array}$ & $\begin{array}{l}12.2 \\
24.4 \\
36.6 \\
48.8 \\
61.0 \\
73.2 \\
85.3 \\
97.5 \\
109.7 \\
121.9 \\
134.1 \\
146.3 \\
158.5 \\
170.7 \\
132.9 \\
195.1 \\
207.3 \\
219.5 \\
231.6 \\
243.8 \\
256.0 \\
268.2 \\
280.4 \\
292.6 \\
304.8 \\
317.0 \\
329.2 \\
341.4 \\
353.6 \\
365.8 \\
378.0 \\
390.1 \\
402.3 \\
414.5 \\
426.7 \\
438.9 \\
451.1\end{array}$ & $\begin{array}{l}10.48 \\
10.59 \\
10.71 \\
10.89 \\
11.13 \\
11.42 \\
11.80 \\
12.26 \\
12.70 \\
13.12 \\
13.52 \\
13.83 \\
14.30 \\
14.77 \\
15.22 \\
15.68 \\
16.14 \\
16.61 \\
17.09 \\
17.52 \\
17.95 \\
18.44 \\
18.85 \\
19.06 \\
19.29 \\
19.51 \\
19.71 \\
19.92 \\
20.10 \\
20.26 \\
20.42 \\
20.56 \\
20.76 \\
21.02 \\
21.26 \\
21.49 \\
21.72\end{array}$ & $\begin{array}{r}15.2 \\
27.4 \\
39.6 \\
51.8 \\
64.0 \\
76.2 \\
88.4 \\
100.6 \\
112.8 \\
125.0 \\
137.2 \\
149.4 \\
161.5 \\
173.7 \\
185.9 \\
198.1 \\
210.3 \\
222.5 \\
234.7 \\
246.9 \\
259.1 \\
271.3 \\
283.5 \\
295.7 \\
307.8 \\
320.0 \\
332.2 \\
344.4 \\
356.6 \\
368.8 \\
381.0 \\
393.2 \\
405.4 \\
417.6 \\
429.8 \\
442.0 \\
454.2\end{array}$ & $\begin{array}{l}10.50 \\
10.61 \\
10.75 \\
10.94 \\
11.20 \\
11.50 \\
11.91 \\
12.37 \\
12.81 \\
13.22 \\
13.55 \\
13.94 \\
14.41 \\
14.89 \\
15.33 \\
15.80 \\
16.26 \\
16.73 \\
17.21 \\
17.63 \\
18.05 \\
18.59 \\
18.90 \\
19.12 \\
19.35 \\
19.56 \\
19.75 \\
19.97 \\
20.15 \\
20.30 \\
20.45 \\
20.60 \\
20.83 \\
21.08 \\
21.32 \\
21.55 \\
21.76\end{array}$ \\
\hline
\end{tabular}




\section{APPENDIX B}

TABLE B-II (Cont)

\begin{tabular}{|c|c|c|c|c|c|c|c|}
\hline$\underset{N}{\text { DEPIH }}$ & TERP & DEPIH & $\underset{C}{\text { TEP }}$ & $\begin{array}{c}\text { DEPTH } \\
M\end{array}$ & $\underset{C}{\text { TERP }}$ & $\begin{array}{c}\text { DEPIH } \\
\mathfrak{M}\end{array}$ & TEP \\
\hline $\begin{array}{l}603.5 \\
615.7 \\
457.2 \\
469.4 \\
481.6 \\
493.8 \\
506.0 \\
518.2 \\
530.4 \\
542.5 \\
554.7 \\
566.9 \\
579.1 \\
591.3\end{array}$ & $\begin{array}{l}24.57 \\
24.83 \\
21.82 \\
22.00 \\
22.14 \\
22.26 \\
22.39 \\
22.54 \\
22.81 \\
23.20 \\
23.49 \\
23.74 \\
23.91 \\
24.17\end{array}$ & $\begin{array}{l}606.6 \\
618.7 \\
460.2 \\
472.4 \\
484.6 \\
496.8 \\
509.0 \\
521.2 \\
533.4 \\
545.6 \\
557.8 \\
570.0 \\
582.2 \\
594.4\end{array}$ & $\begin{array}{l}24.63 \\
24.84 \\
21.87 \\
22.05 \\
22.16 \\
22.30 \\
22.44 \\
22.59 \\
22.90 \\
23.29 \\
23.57 \\
23.79 \\
23.94 \\
24.20\end{array}$ & $\begin{array}{l}609.6 \\
463.3 \\
475.5 \\
487.7 \\
499.9 \\
512.1 \\
524.3 \\
536.4 \\
548.6 \\
560.8 \\
573.0 \\
585.2 \\
597.4\end{array}$ & $\begin{array}{l}24.66 \\
21.92 \\
22.07 \\
22.19 \\
22.33 \\
22.46 \\
22.65 \\
23.01 \\
23.37 \\
23.63 \\
23.83 \\
24.00 \\
24.29\end{array}$ & $\begin{array}{l}612.6 \\
466.3 \\
478.5 \\
490.7 \\
502.9 \\
515.1 \\
527.3 \\
539.5 \\
551.7 \\
563.9 \\
576.1 \\
588.3 \\
600.5\end{array}$ & $\begin{array}{l}24.86 \\
21.97 \\
22.10 \\
22.22 \\
22.36 \\
22.50 \\
22.71 \\
23.10 \\
23.43 \\
23.68 \\
23.87 \\
24.10 \\
24.37\end{array}$ \\
\hline
\end{tabular}




\section{APPENDIX B (Cont) \\ TABLE B-III}

WELL NO. 14270 WILLIAMS 1 - EDEN ERIE CO., NEW YORK 7-10-79

LATITUDE 7600. FEET $S$ of 42.40 .0$.

LONGITUDE 5200. FEET $W$ of 78.55 .0 .

ELEVATION 765. FEET

\begin{tabular}{|c|c|c|c|c|c|c|c|}
\hline$\underset{\mathrm{M}}{\mathrm{DEPTH}}$ & $\underset{\mathrm{C}}{\mathrm{TER} P \mathrm{P}}$ & $\underset{H}{D E P I H}$ & $\underset{\mathrm{C}}{\mathrm{TEP}}$ & $\underset{\mathbb{I}}{\mathrm{DEPIH}}$ & $\underset{\mathrm{C}}{\mathrm{TEP}}$ & $\underset{\mathrm{H}}{\mathrm{DEPTH}}$ & TETP \\
\hline $\begin{array}{r}15.5 \\
76.5 \\
137.5 \\
198.4 \\
259.4 \\
320.3 \\
381.3 \\
442.3 \\
503.2 \\
564.2 \\
625.1 \\
686.1\end{array}$ & $\begin{array}{l}10.83 \\
11.81 \\
13.36 \\
15.47 \\
17.54 \\
18.42 \\
19.42 \\
20.31 \\
21.66 \\
21.96 \\
22.62 \\
22.93\end{array}$ & $\begin{array}{r}30.8 \\
91.7 \\
152.7 \\
213.7 \\
274.6 \\
335.6 \\
396.5 \\
457.5 \\
518.5 \\
579.4 \\
640.4\end{array}$ & $\begin{array}{l}11.00 \\
11.99 \\
14.34 \\
16.38 \\
17.77 \\
18.60 \\
19.72 \\
20.47 \\
21.71 \\
22.13 \\
22.75\end{array}$ & $\begin{array}{r}46.0 \\
107.0 \\
167.9 \\
228.9 \\
289.9 \\
350.8 \\
411.8 \\
472.7 \\
533.7 \\
594.7 \\
655.6\end{array}$ & $\begin{array}{l}11.39 \\
12.58 \\
14.66 \\
17.06 \\
17.98 \\
18.83 \\
19.95 \\
20.70 \\
21.80 \\
22.24 \\
22.86\end{array}$ & $\begin{array}{r}61.3 \\
122.2 \\
183.2 \\
244.1 \\
305.1 \\
366.1 \\
427.0 \\
488.0 \\
548.9 \\
609.9 \\
670.9\end{array}$ & $\begin{array}{l}11.66 \\
13.04 \\
14.99 \\
17.32 \\
18.24 \\
19.14 \\
20.13 \\
21.17 \\
21.90 \\
22.50 \\
22.91\end{array}$ \\
\hline
\end{tabular}


APPENDIX B (Cont)

TABLE B-IV

WELL NO. 14269 LARDO 1 - EDEN ERIE CO., NEW YORK 7-9-79

LATITUDE 3160. FEET S of 42. 40. 0. LONGITUDE 2200. FEET $W$ of 78. 55. 0. ELEVATION 782. FEET

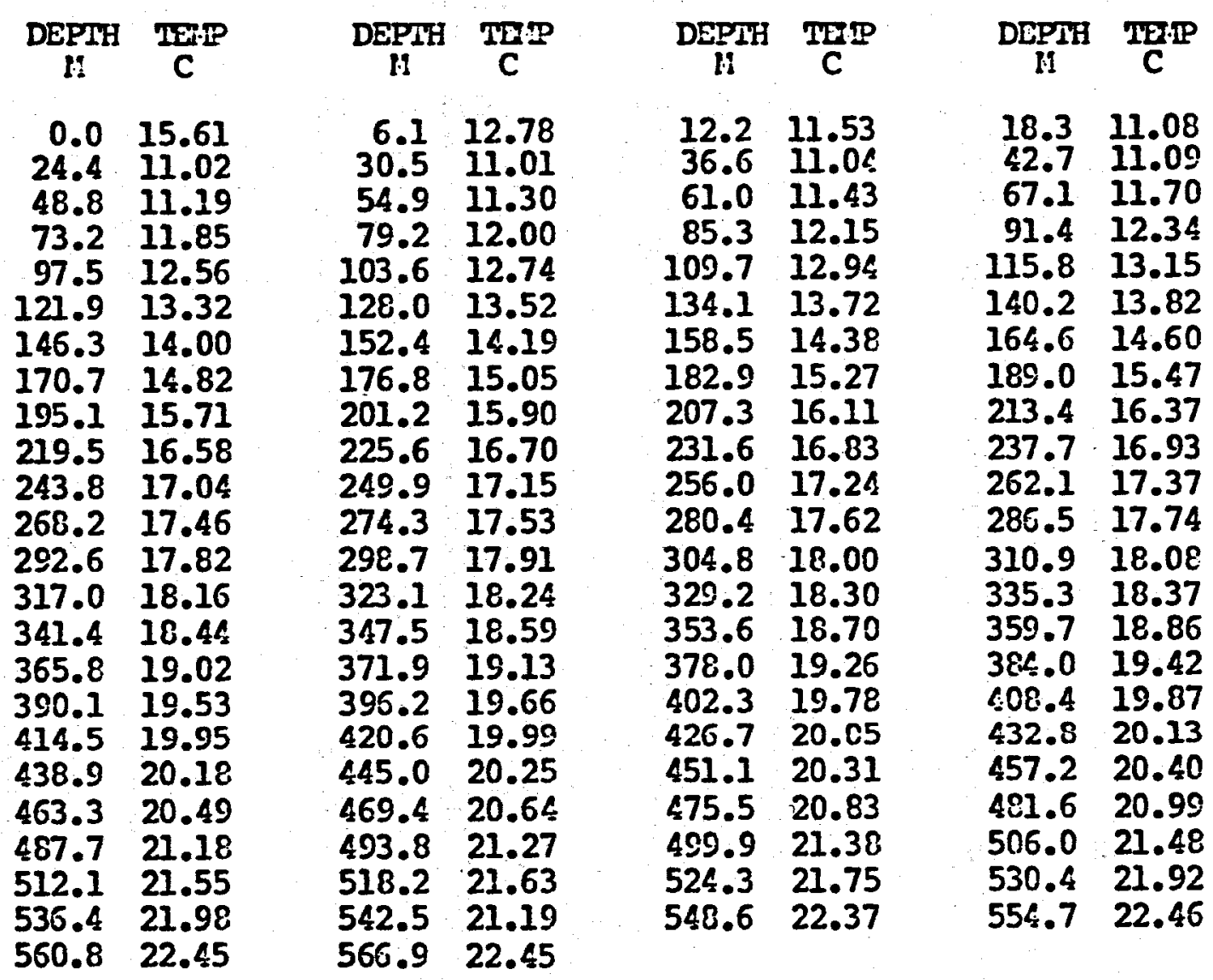


APPENDIX B (Cont)

TABLE B-V

WELL NO. 14310 ANDERSON 1 - BUSTI CHAUTAUQUA CO., NEW YORK 8-24-79

LATITUDE 8760. FEET $S$ of 42. 5. 0.

LONGITUDE 4350 . FEET $W$ of 79. 17. 30.

ELEVATION 1510. FEET

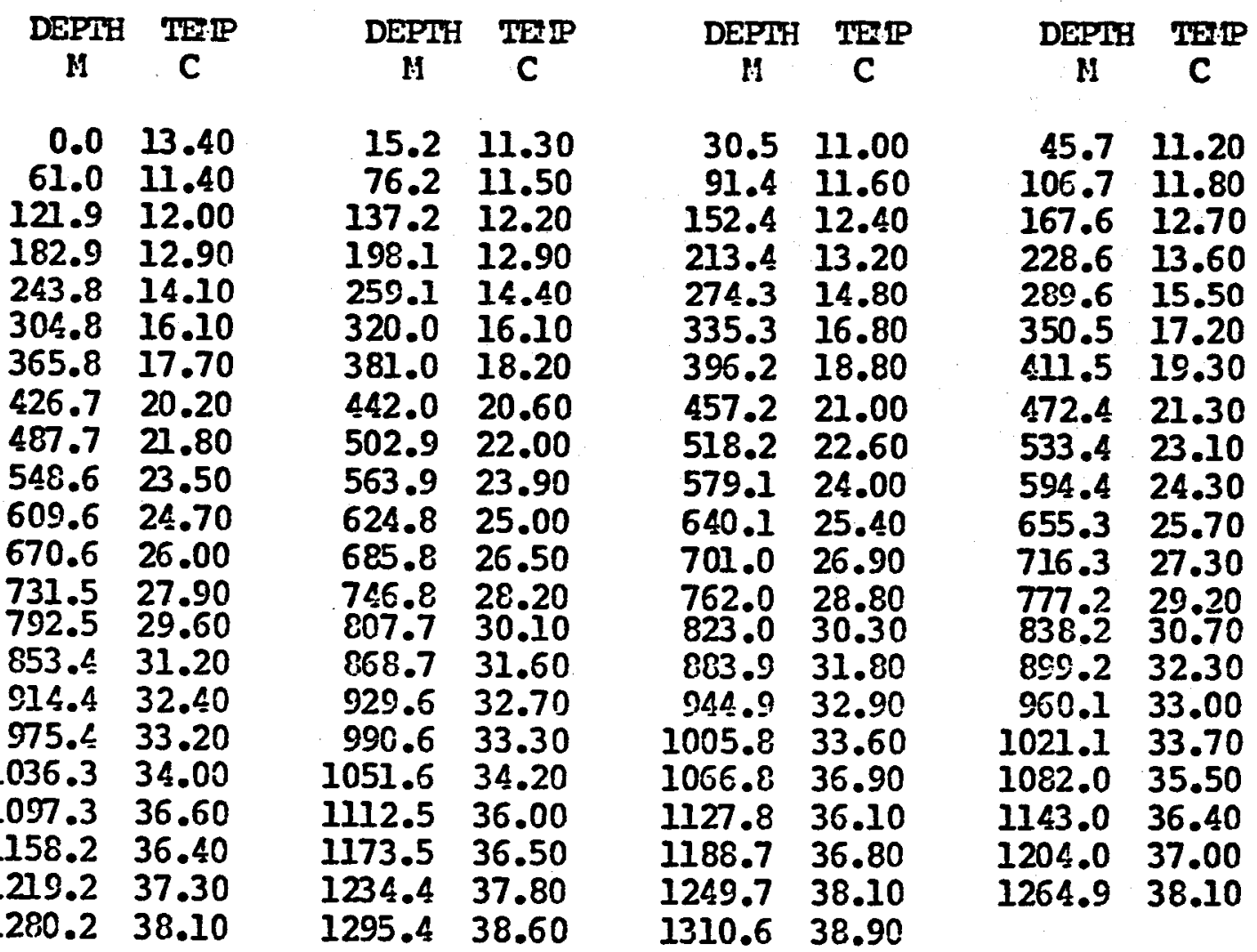


APPENDIX B (Cont)

TABLE B-VI

WELL NO. 14324 E AURORA HIGH SCHOOL ERIE CO., NEW YORK 8-9-79

LATITUDE 6800. FEET $S$ of 42.45 .0 . LONGITUDE 10875. FEET W of 78. 35. 0. ELEVATION .1140. FEET

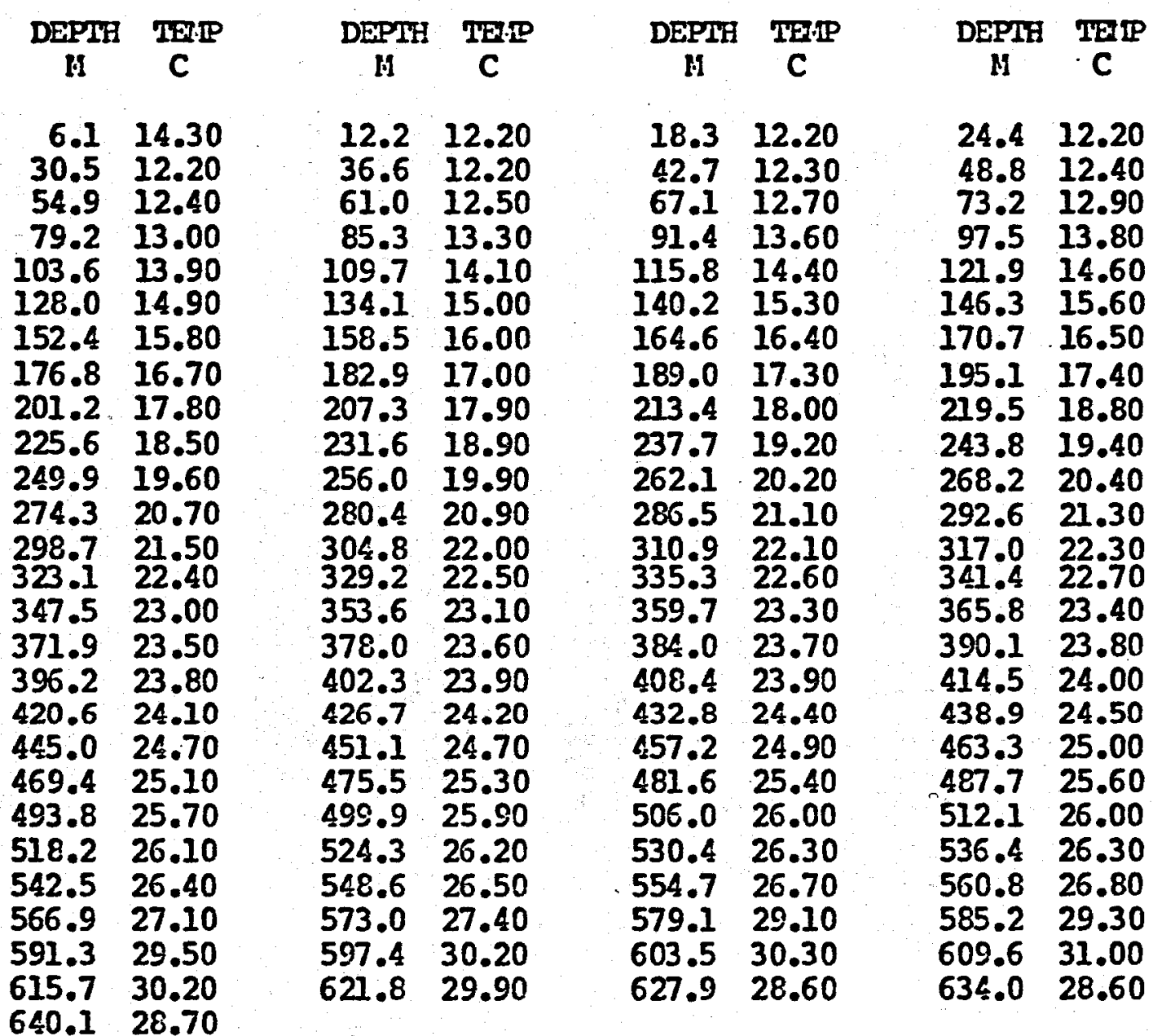


APPENDIX B (Cont)

TABLE B-VII

WELL N0. 14365 NELSON L-A - BUSTI CHAUTAUQUA CO., NEW YORK 8-6-79

LATITUDE 1300. FEET S of 42. 5.0 .

LONGITUDE 5500. FEET $W$ of 79. 20. 0.

ELEVATION 1395. FEET

\begin{tabular}{|c|c|c|c|c|c|c|c|}
\hline $\begin{array}{c}\text { DEPTH } \\
M\end{array}$ & $\begin{array}{c}\text { TER.PP } \\
\mathrm{C}\end{array}$ & $\begin{array}{c}\text { DEPIH } \\
\mathrm{H}\end{array}$ & $\begin{array}{c}\text { TEXPP } \\
\text { C }\end{array}$ & $\begin{array}{c}\text { DEPIH } \\
\text { HI }\end{array}$ & $\underset{\mathrm{C}}{\text { TYETP }}$ & $\underset{M}{\text { DEPTH }}$ & $\underset{C}{\text { IFPP }}$ \\
\hline $\begin{array}{r}15.2 \\
76.2 \\
137.2 \\
198.1 \\
259.1 \\
320.0 \\
381.0 \\
442.0 \\
502.9 \\
563.9 \\
624.8 \\
685.8 \\
746.8 \\
807.7 \\
868.7 \\
929.6 \\
990.6 \\
1051.6 \\
1112.5 \\
1173.5\end{array}$ & $\begin{array}{l}18.10 \\
24.30 \\
20.80 \\
22.10 \\
23.90 \\
25.60 \\
26.90 \\
28.10 \\
29.30 \\
29.80 \\
30.90 \\
31.40 \\
32.90 \\
34.10 \\
35.80 \\
37.00 \\
37.00 \\
38.10 \\
38.60 \\
38.30 \\
38.50\end{array}$ & $\begin{array}{r}30.5 \\
91.4 \\
152.4 \\
213.4 \\
274.3 \\
335.3 \\
396.2 \\
457.2 \\
518.2 \\
579.1 \\
640.1 \\
701.0 \\
762.0 \\
823.0 \\
883.9 \\
944.9 \\
1005.8 \\
1066.8 \\
1127.8 \\
1188.7 \\
1249.7\end{array}$ & $\begin{array}{l}12.20 \\
22.00 \\
20.70 \\
22.80 \\
24.20 \\
25.80 \\
27.10 \\
28.20 \\
29.50 \\
30.00 \\
31.20 \\
32.00 \\
33.30 \\
34.70 \\
36.00 \\
37.20 \\
37.50 \\
38.10 \\
38.60 \\
38.50 \\
38.90\end{array}$ & $\begin{array}{r}45.7 \\
106.7 \\
167.6 \\
228.6 \\
269.6 \\
350.5 \\
411.5 \\
472.4 \\
533.4 \\
594.4 \\
655.3 \\
716.3 \\
777.2 \\
838.2 \\
899.2 \\
960.1 \\
1021.1 \\
1082.0 \\
1143.0 \\
1204.0\end{array}$ & $\begin{array}{l}23.60 \\
20.50 \\
20.90 \\
23.30 \\
24.70 \\
26.60 \\
27.30 \\
28.60 \\
29.40 \\
30.20 \\
31.00 \\
32.50 \\
33.60 \\
35.10 \\
35.70 \\
37.30 \\
37.50 \\
38.20 \\
38.50 \\
38.10\end{array}$ & $\begin{array}{r}61.0 \\
121.9 \\
182.9 \\
243.8 \\
304.8 \\
365.8 \\
426.7 \\
487.7 \\
548.6 \\
609.6 \\
670.6 \\
731.5 \\
792.5 \\
853.4 \\
914.4 \\
975.4 \\
1036.3 \\
1097.3 \\
1158.2 \\
1219.2\end{array}$ & $\begin{array}{l}25.30 \\
20.60 \\
21.10 \\
24.10 \\
25.10 \\
26.70 \\
27.70 \\
28.80 \\
29.80 \\
30.70 \\
31.40 \\
32.80 \\
34.00 \\
35.50 \\
37.00 \\
37.40 \\
38.10 \\
38.40 \\
38.40 \\
38.40\end{array}$ \\
\hline
\end{tabular}




\section{APPENDIX B (Cont)}

TABLE B-VIII

WELL NO. 14172 HONEOYE - BRISTOL ONTARIO CO., NEW YORK 7-7-79

LATITUDE 12400. FEET $S$ of 42.50 .0 .

LONGITUDE 1100. FEET W Of 77. 27. 30.

ELEVATION 1477. FEET

\begin{tabular}{|c|c|c|c|c|c|c|c|}
\hline$\underset{M}{\text { DEPIH }}$ & $\frac{\text { TEIPP }}{\mathrm{C}}$ & $\begin{array}{c}\text { DEPTH } \\
\mathrm{H}\end{array}$ & $\begin{array}{c}\text { TEESP } \\
\mathrm{C}\end{array}$ & $\begin{array}{c}\text { DEPTH } \\
M\end{array}$ & ${ }_{\mathrm{C}}^{\mathrm{TEX} P}$ & $\underset{N}{\text { DEPTH }}$ & $\underset{\mathrm{CE}}{\mathrm{C}}$ \\
\hline $\begin{array}{r}6.1 \\
30.5 \\
54.9 \\
79.2 \\
103.6 \\
128.0 \\
152.4 \\
176.8 \\
201.2 \\
225.6 \\
249.9 \\
274.3 \\
298.7 \\
323.1 \\
347.5 \\
371.9 \\
396.2 \\
420.6\end{array}$ & $\begin{array}{r}10.11 \\
9.77 \\
10.10 \\
10.67 \\
11.08 \\
11.91 \\
12.47 \\
13.00 \\
13.63 \\
14.16 \\
14.65 \\
15.33 \\
15.77 \\
16.36 \\
16.97 \\
17.60 \\
18.35 \\
18.66\end{array}$ & $\begin{array}{r}12.2 \\
36.6 \\
61.0 \\
85.3 \\
109.7 \\
134.1 \\
158.5 \\
182.9 \\
207.3 \\
231.6 \\
256.0 \\
280.4 \\
304.8 \\
329.2 \\
353.6 \\
378.0 \\
402.3 \\
426.7\end{array}$ & $\begin{array}{r}9.42 \\
9.86 \\
10.24 \\
10.79 \\
11.20 \\
12.05 \\
12.60 \\
13.17 \\
13.77 \\
14.23 \\
14.80 \\
15.45 \\
15.92 \\
16.50 \\
17.13 \\
17.82 \\
18.45 \\
18.72\end{array}$ & $\begin{array}{r}18.3 \\
42.7 \\
67.1 \\
91.4 \\
115.8 \\
140.2 \\
164.6 \\
189.0 \\
213.4 \\
237.7 \\
262.1 \\
286.5 \\
310.9 \\
335.3 \\
359.7 \\
384.0 \\
408.4\end{array}$ & $\begin{array}{r}9.54 \\
9.93 \\
10.37 \\
10.90 \\
11.28 \\
12.19 \\
12.74 \\
13.37 \\
13.88 \\
14.37 \\
14.99 \\
15.54 \\
16.07 \\
16.67 \\
17.28 \\
18.00 \\
18.54\end{array}$ & $\begin{array}{r}24.4 \\
48.8 \\
73.2 \\
97.5 \\
121.9 \\
146.3 \\
170.7 \\
195.1 \\
219.5 \\
243.8 \\
268.2 \\
292.6 \\
317.0 \\
341.4 \\
365.8 \\
390.1 \\
414.5\end{array}$ & $\begin{array}{r}9.65 \\
10.04 \\
10.52 \\
10.97 \\
11.41 \\
12.30 \\
12.88 \\
13.49 \\
14.02 \\
14.53 \\
15.13 \\
15.61 \\
16.22 \\
16.82 \\
17.44 \\
18.20 \\
18.61\end{array}$ \\
\hline
\end{tabular}


APPENDIX B (Cont)

TABLE B-IX

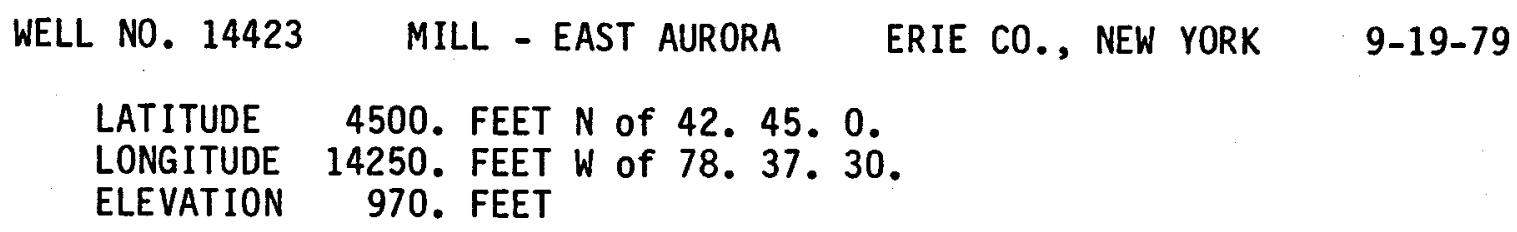

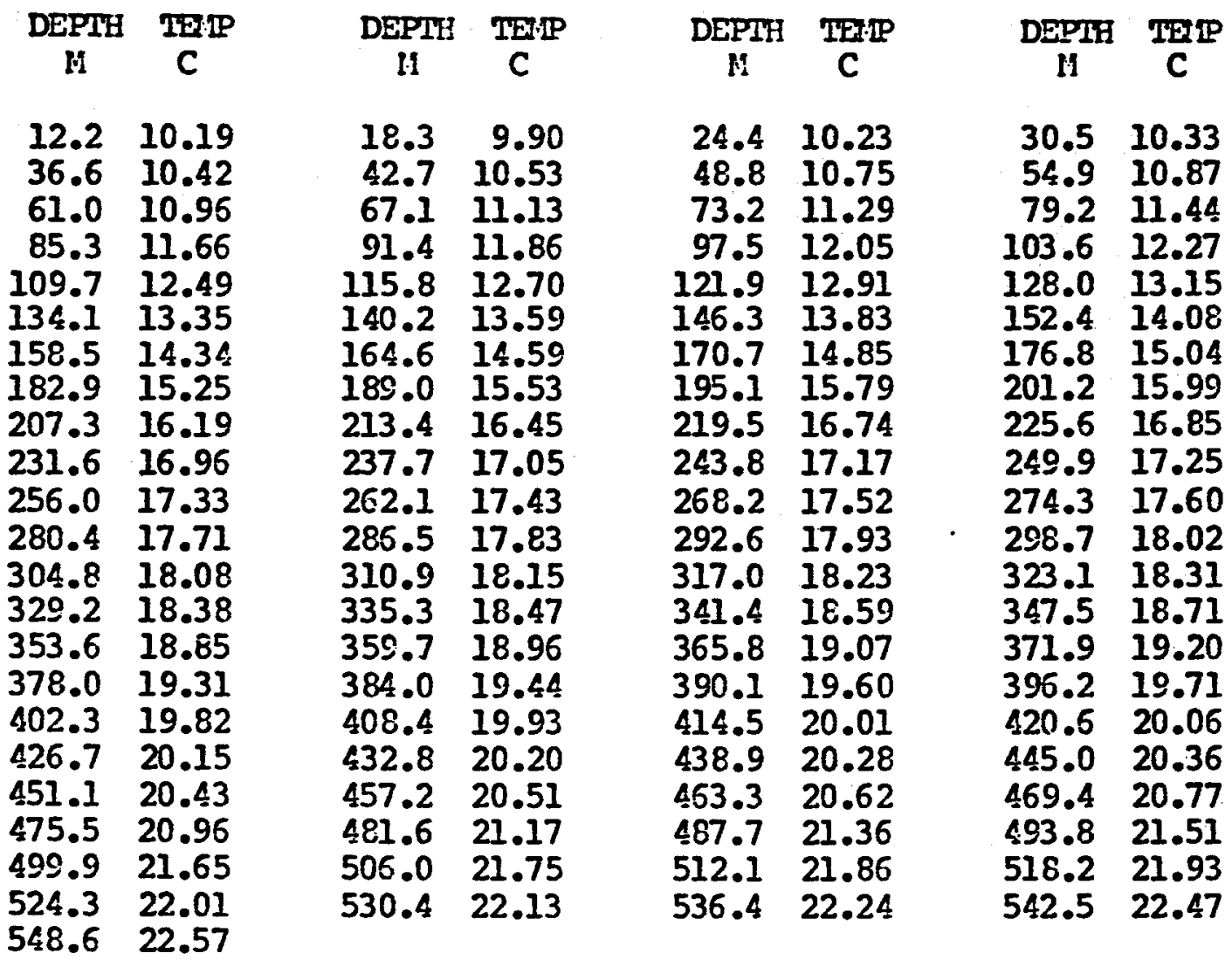




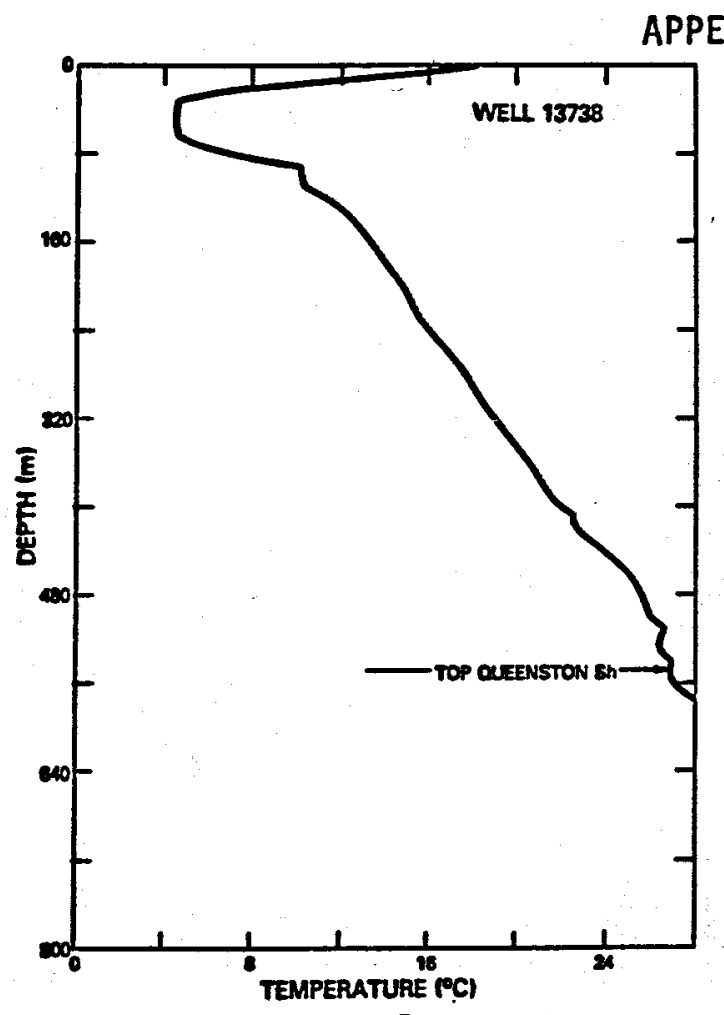

Fig. B-1 .

Temperature-depth profile for Wel1 13738.

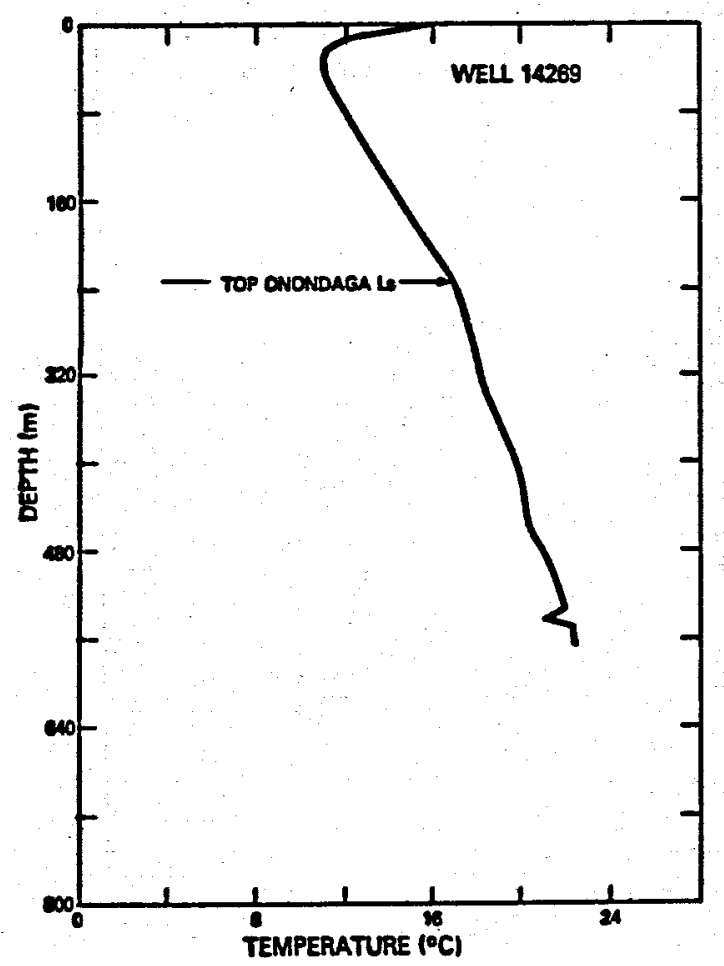

Fig. B-3.

Temperature-depth profile for Well 14269.

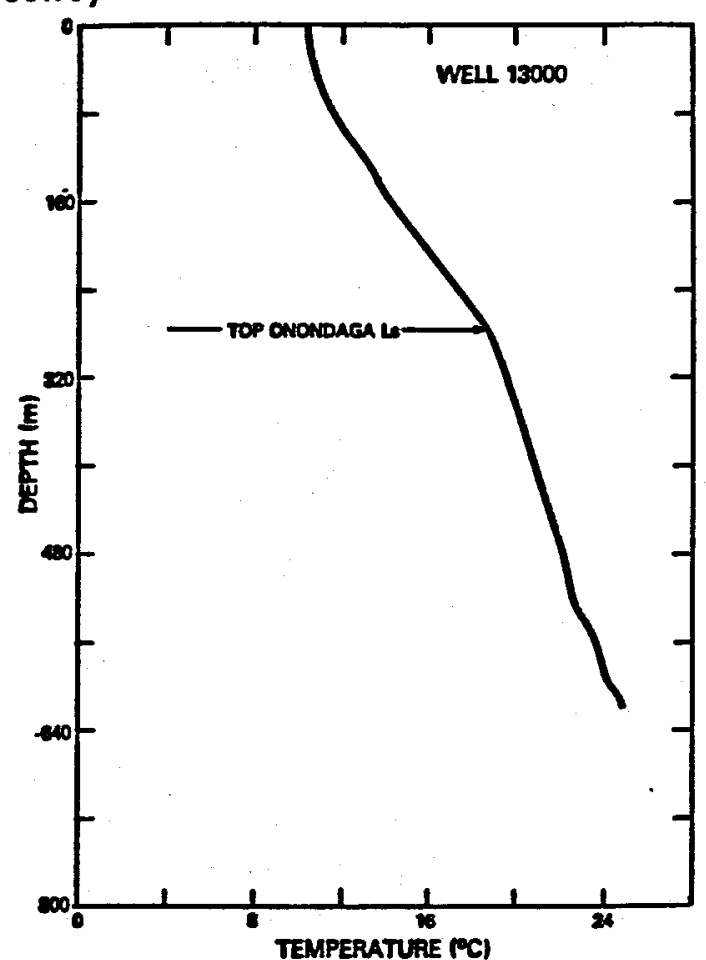

Fig. B-2.

Temperature-depth profile for We11 13000.

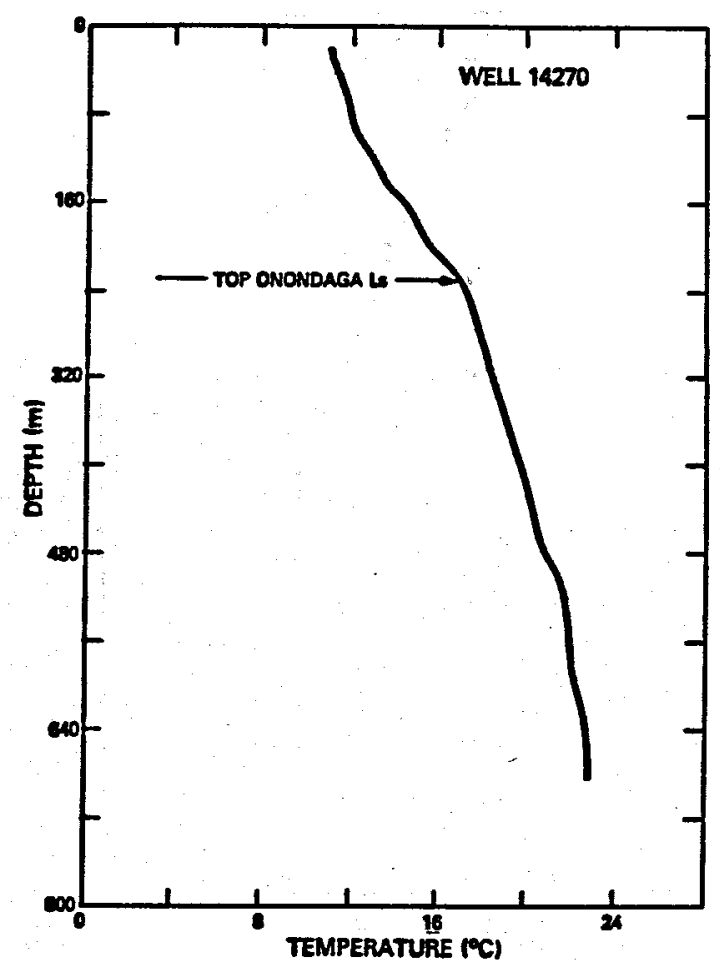

Fig. B-4.

Temperature-depth profile for Wel1 14270. 
APPENDIX B (Cont)

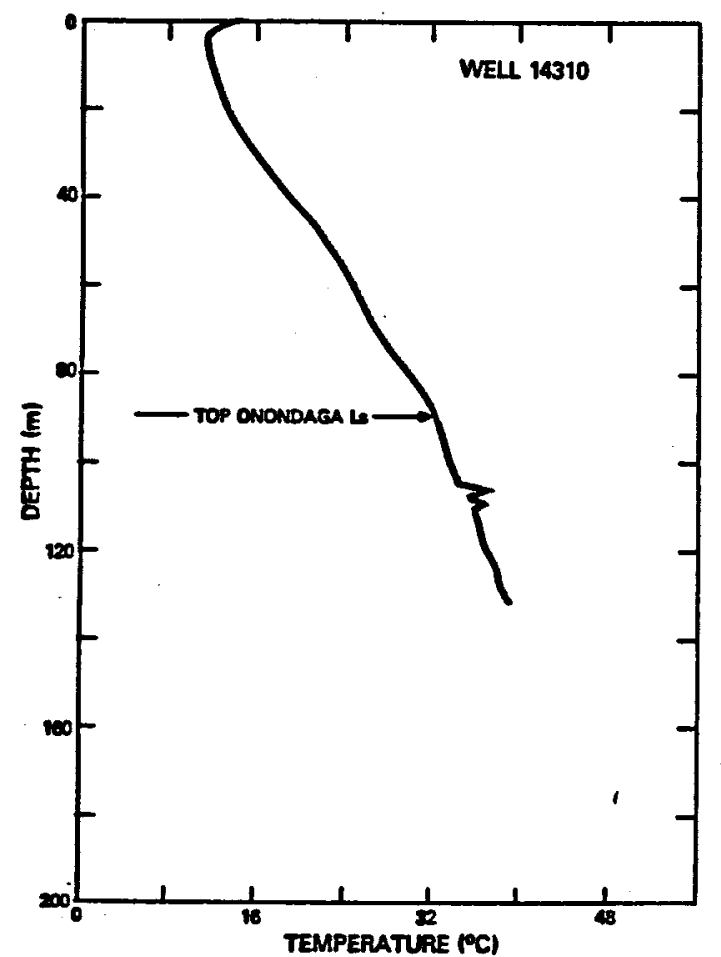

Fig. B-5.

Temperature-depth profile for Well 14310.

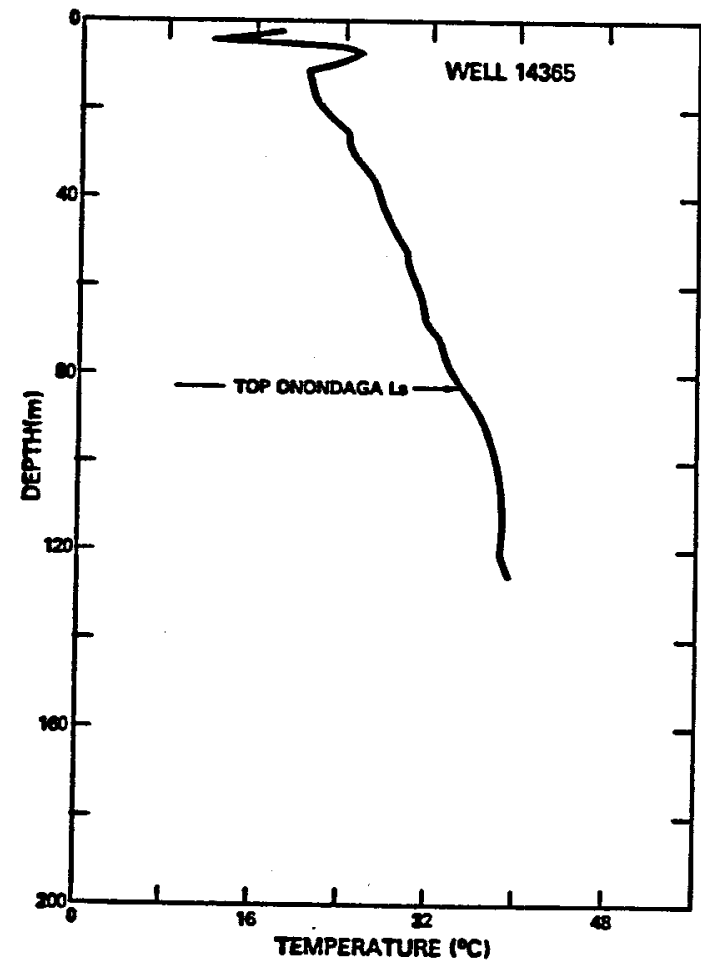

Fig. B-7.

Temperature-depth profile for We11 14365.

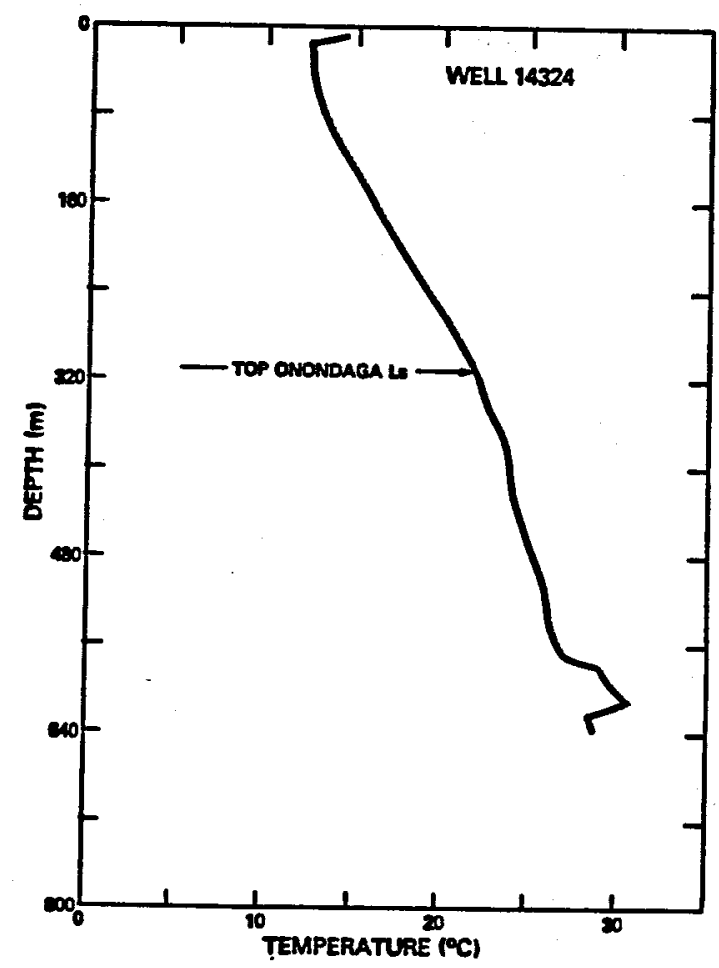

Fig. B-6.

Temperature-depth profile for Well 14324.

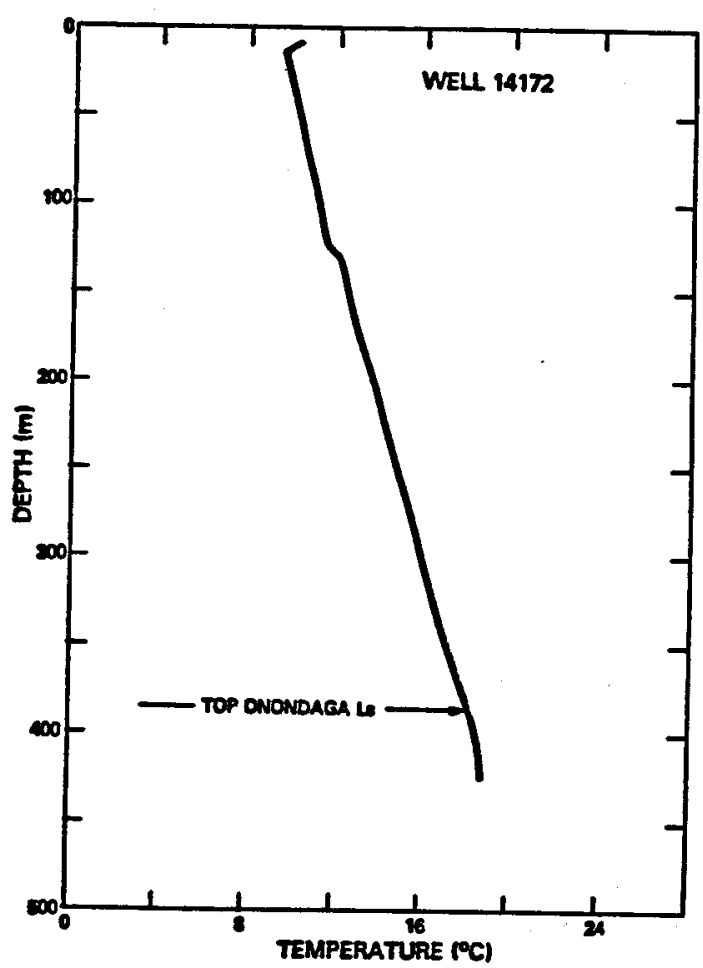

Fig. B-8.

Temperature-depth profile for Well 14172. 


\section{APPENDIX B (Cont)}

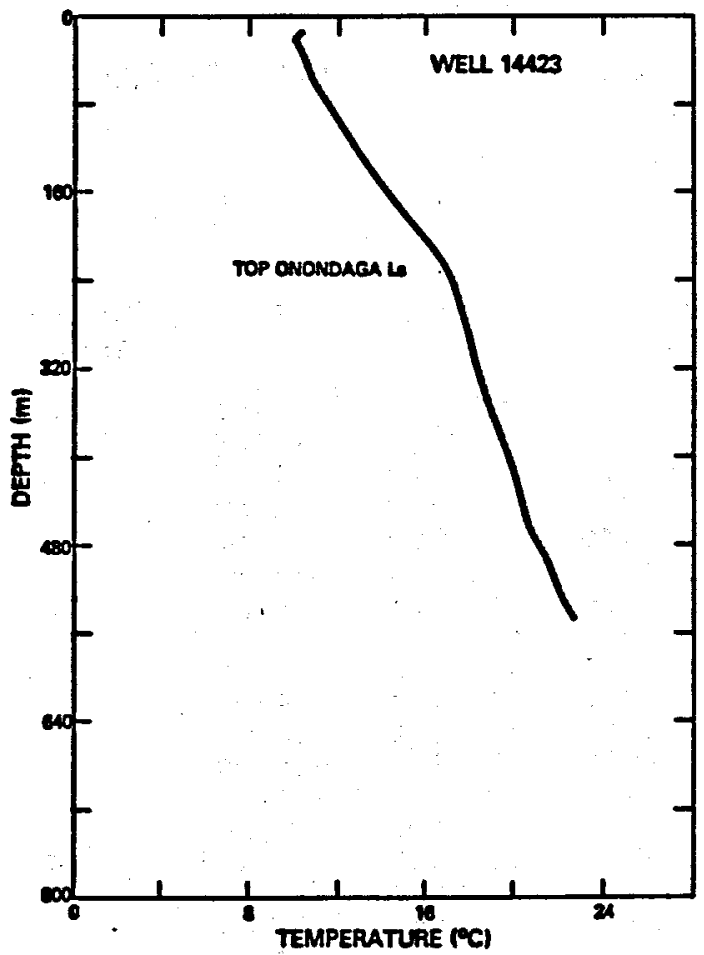

Fig. B-9.

Temperature-depth profile for Wel1 14423. 
APPENDIX C

TOPS OF FORMATION DATA

WELL DATA FOR STRUCTURE CONTOUR MAPS

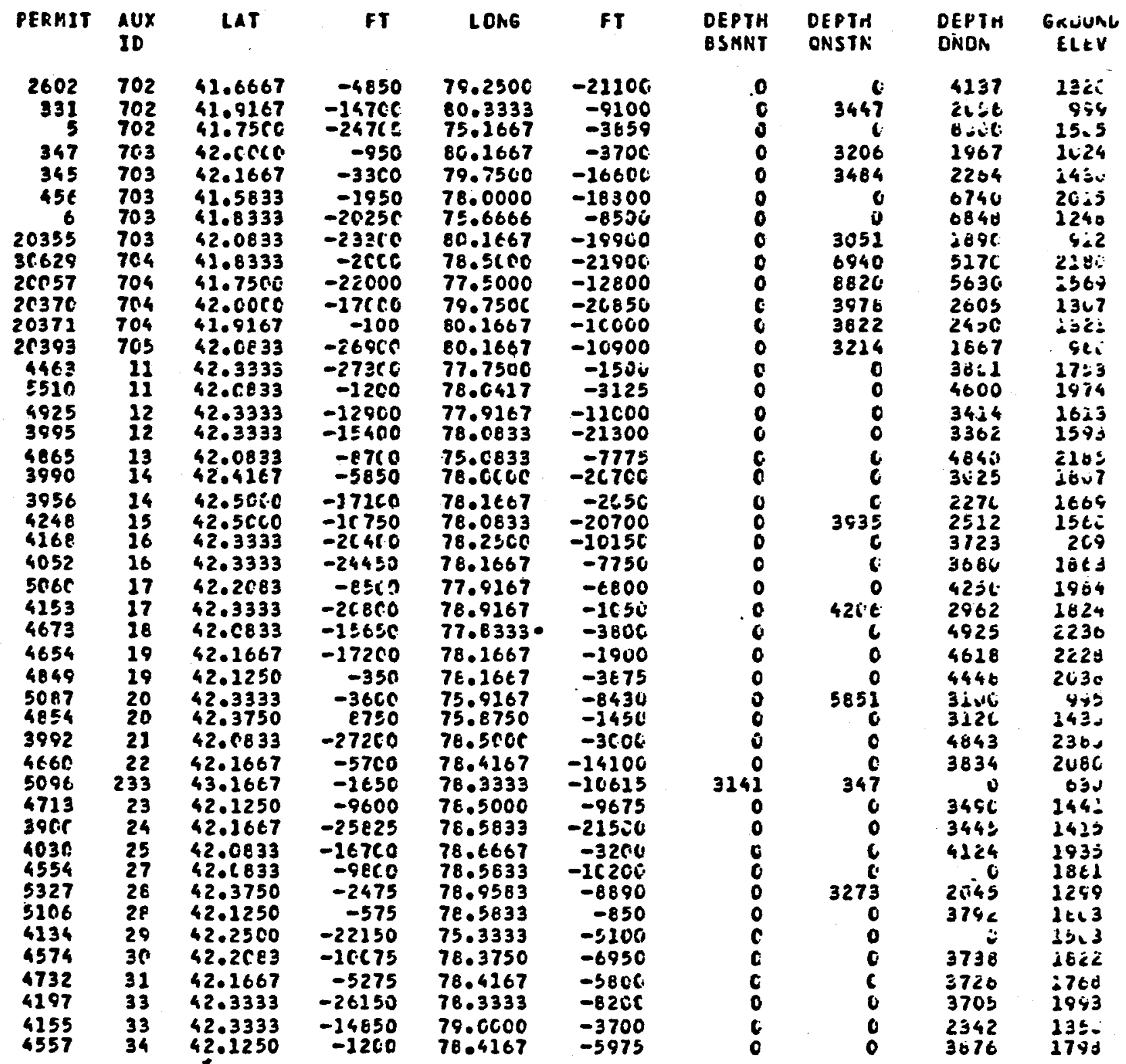

LAT,LONG ARE GIVEN AS DECIKAL DEGREES AND FEET.

POSITIVE VALUES INOICATE A NCRTHERLY UR EASTERLY DISPLACEMENT.

NEGATIVE VALUES IHOICATE SCUTHERLY DR WESTERLY DISFLACERENT. AUXILIILRY IO'S BEGINNIAG WITH 7 INEICATE PENNA KELLS. 
APPENDIX C (Cont)

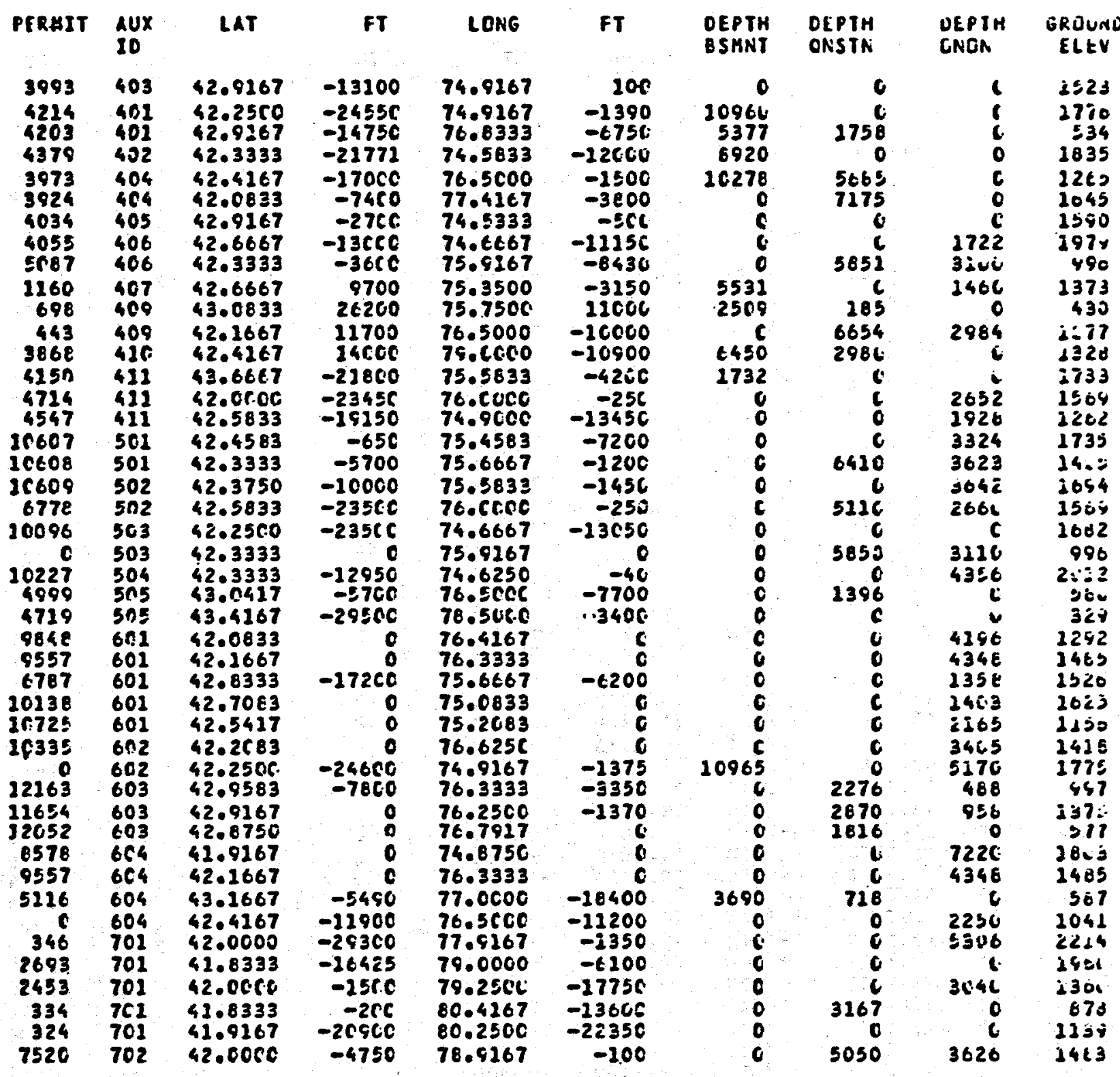

LAT,LONG ARE GIVEN AS DECIRAL DEGREES AND FETT. POSITIVE VALUES INOICATE NCRTHERLY DR EASTERLY DISPLACEMENT. NEgATIVE VALUES INOICATE SCUTHERLY OR MESTERLY DISPLACERENT. AUXILLIARY IOES BEGINNIAG WITH 7 INOICATE PENNA NELLS: 
APPENDIX C (Cont)

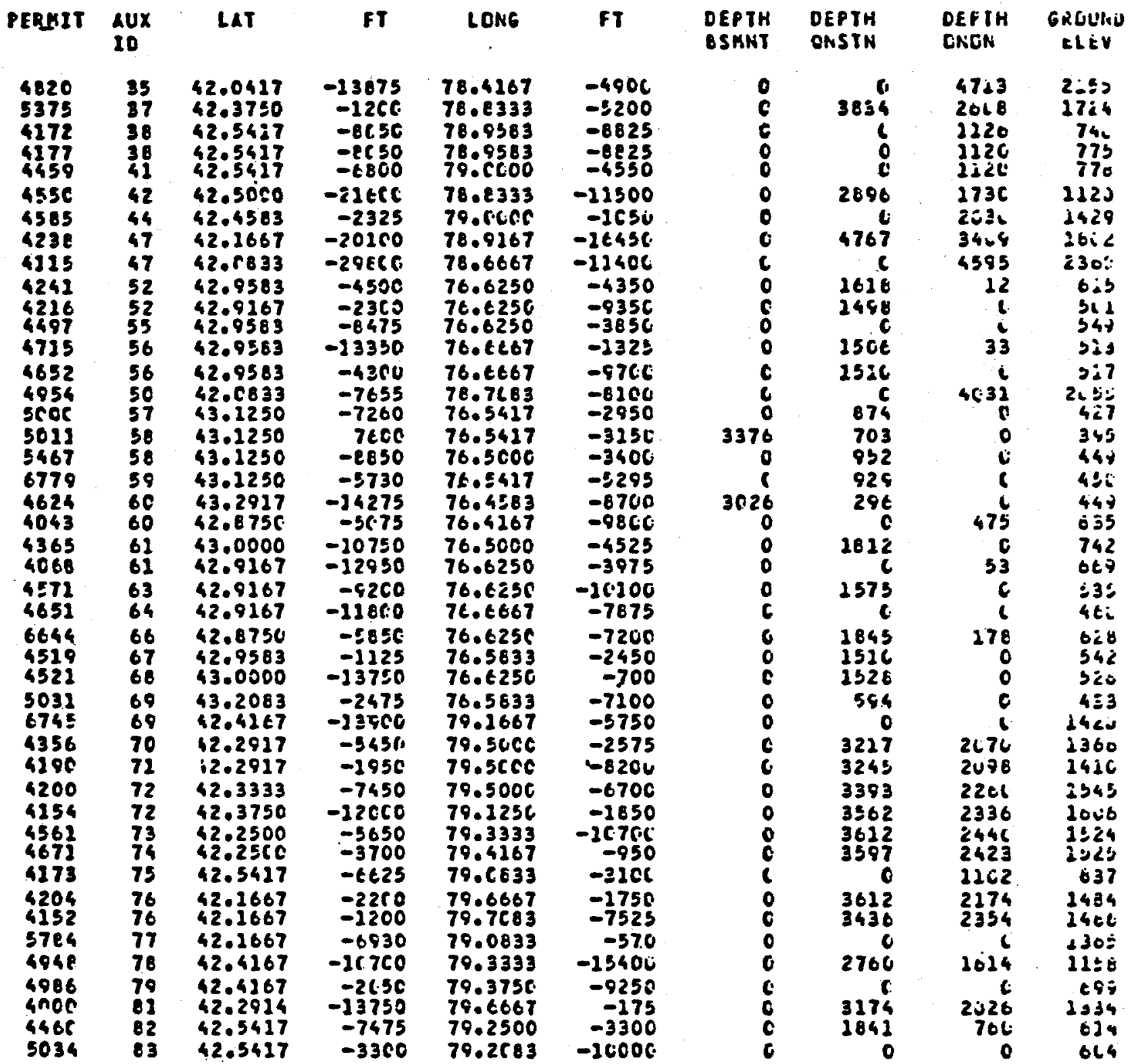

LAT,LCNG RRE GIVEN AS DECIYAL DEGREES AND FEET.

POSITIVE VALUES JNOICATE A NORTHERLY OR EASTERLY DISPLACEMENT. REGATIVE VALUTS INDICATE SCUTHERLY OR YESTERLY DISPLACEMERT. IUXILLILRY IO IS BEGINKING KITH 7 INOICATE PENWA NELLS. 
APPENDIX C (Cont)

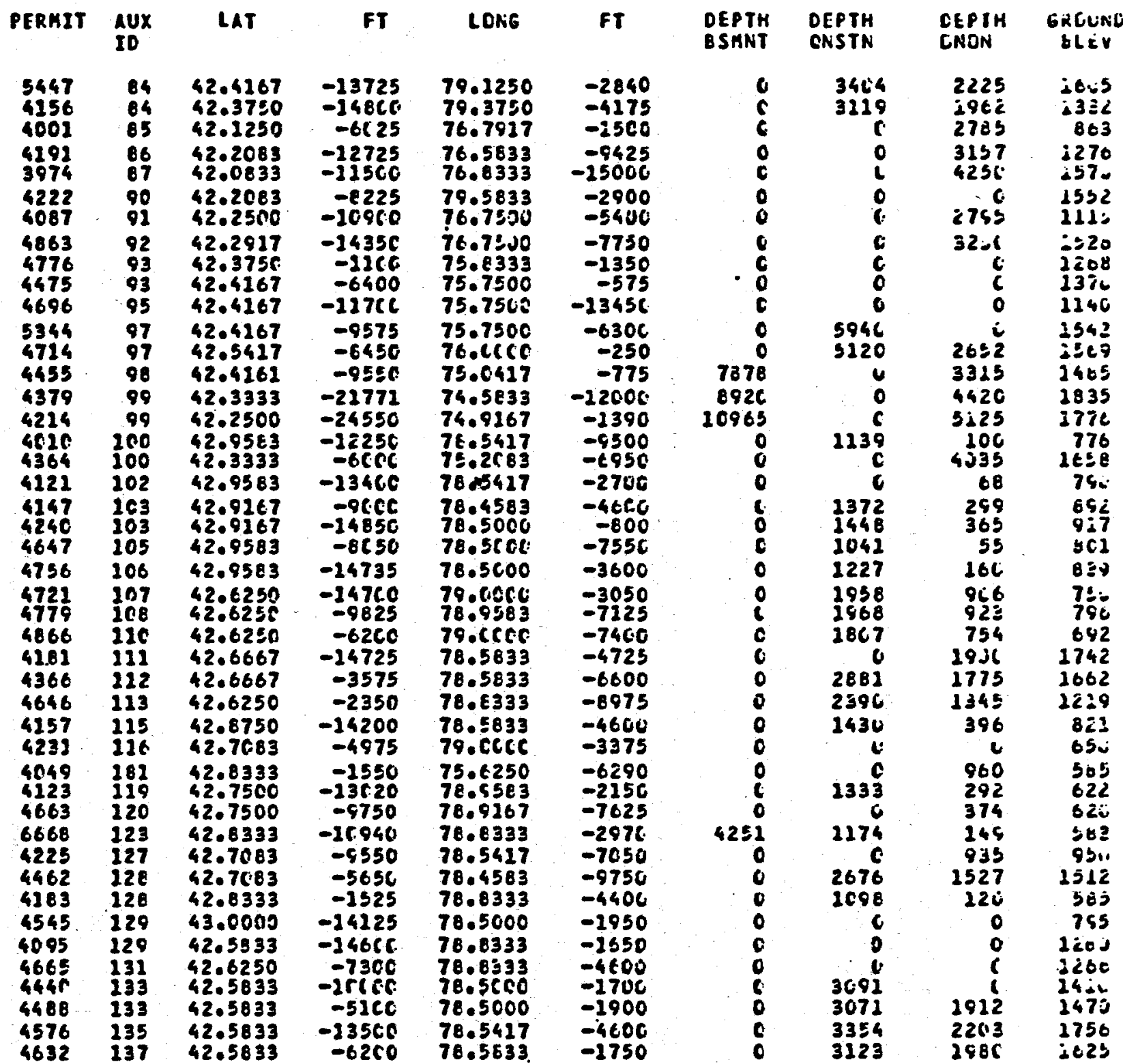

LAT,LONG ARE GIVEN AS DECIMAL OEGREES AND FEET:

POSITIVE VALUES INDICATE A NORTHERLY OR EASTERIY DISPLACERENT. NEGATIVE VALLES INDICATE SCUTHERLY DR WESTERLY DISPLACEMENT. AUXILLIARY IO'S BEGINNING WITH 7 INOICATE PENAA MELLS. 
APPENDIX C (Cont)

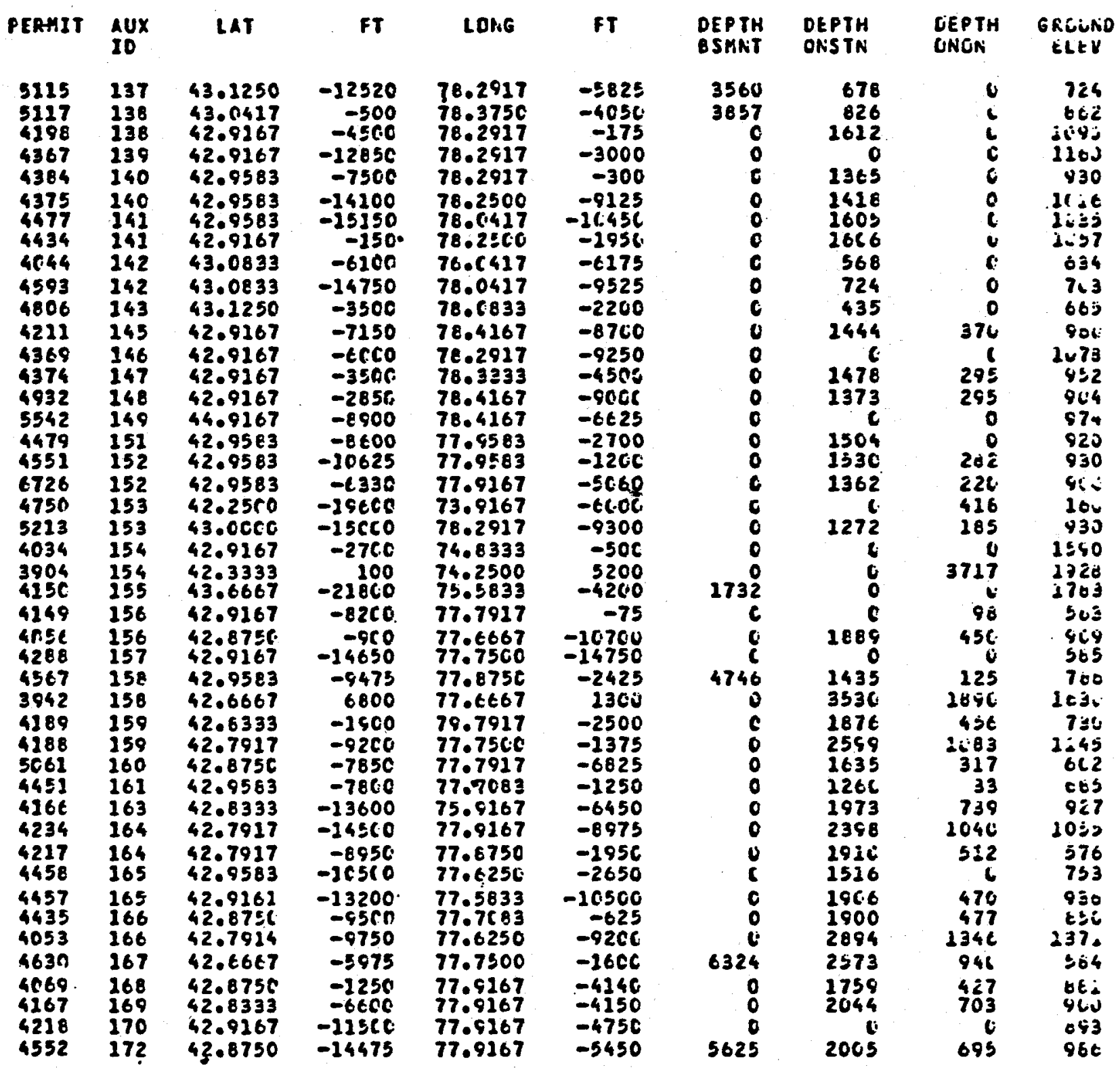

LAT,LONG ARE GIVEN AS DECIYAL OEGREES AND FEET. POSITIVE VALUES INDICATE A NORTHERLY OR EASTERLY OISPLACERENT. NFGATIVE VALUES INDICATE SCUTHERLY OR MESTERLY OISPLACEMENT. AUXILLIARY IC'S BEGINNING WITH 7 INDICATE FENNA WELLS. 
APPENDIX C (Cont)

)

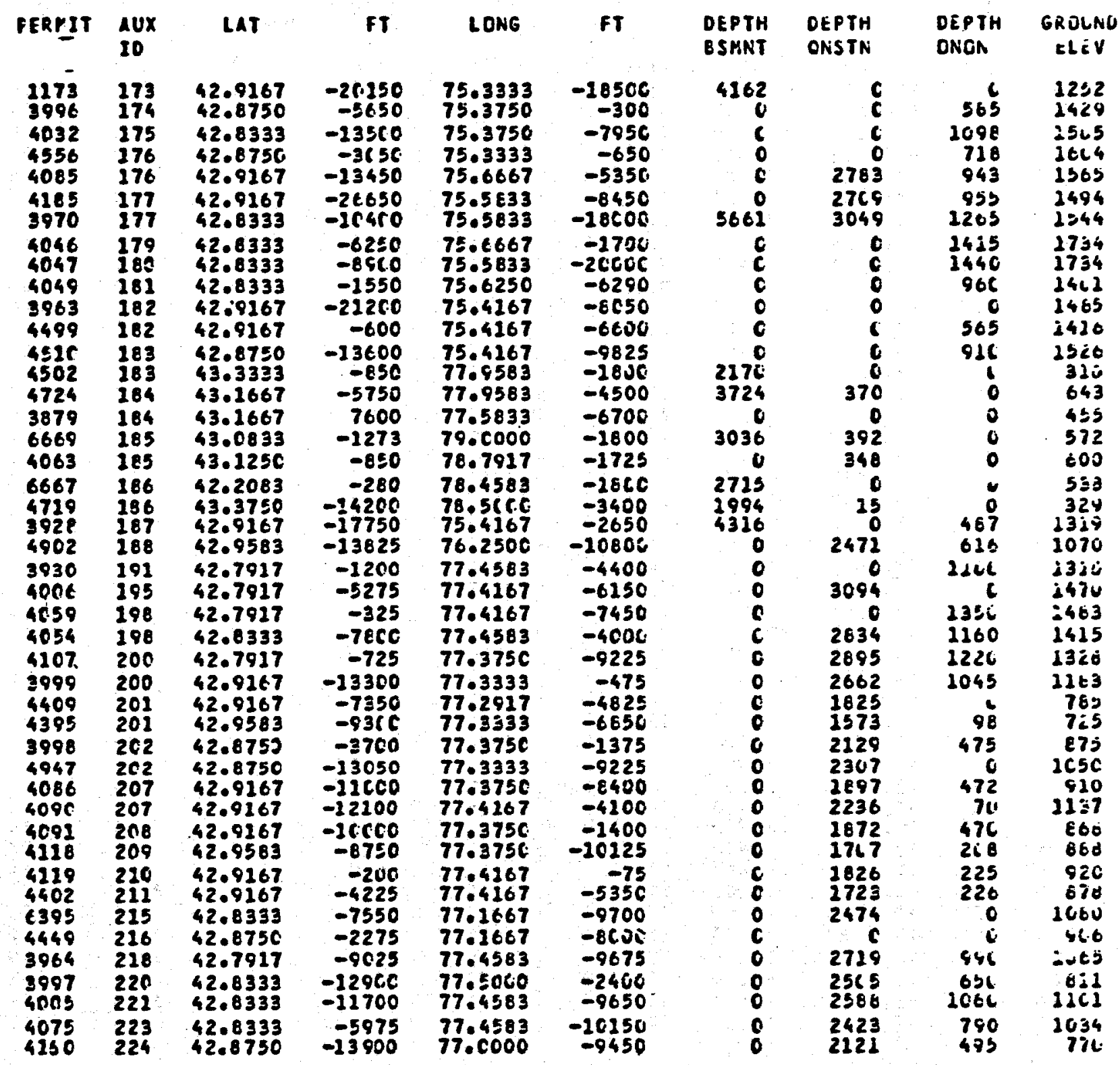

LAT.IDNE ARE GIVEN AS DECIMAL DEGREES ANO FEET.

PESITIVE VALLIES INOICATE A NORTHEKLY OR EASTERLY OISPLACEAENT. NEGATIVE VALUES INDICATE SCUTHERLY OR NESTERLY OISPLACEMERT. AUXILLIART IO'S BEGINNIRG KITH 7 INDICATE PENRA WELLS. 
APPENDIX C (Cont)

\begin{tabular}{|c|c|c|c|c|c|c|c|c|c|}
\hline ERMH & $\operatorname{lu}_{10}$ & LAT & FT & LONG & FT & $\begin{array}{l}\text { DEPTH } \\
\text { BSHNT }\end{array}$ & $\begin{array}{l}\text { DEPTH } \\
\text { CASIN }\end{array}$ & $\begin{array}{l}\text { EP.TH } \\
\text { NON }\end{array}$ & $\begin{array}{l}\text { GROUNU } \\
\text { ELEV }\end{array}$ \\
\hline $\begin{array}{l}5056 \\
3866 \\
4394 \\
4450 \\
4611 \\
4730 \\
4873 \\
5007 \\
4912 \\
5091 \\
8669 \\
5008 \\
4489 \\
4752 \\
4764 \\
4208 \\
5012 \\
4209 \\
4201 \\
4245 \\
4357 \\
4547\end{array}$ & $\begin{array}{l}225 \\
225 \\
226 \\
226 \\
227 \\
227 \\
228 \\
229 \\
229 \\
230 \\
232 \\
232 \\
233 \\
234 \\
235 \\
235 \\
236 \\
236 \\
237 \\
238 \\
238 \\
239\end{array}$ & $\begin{array}{l}42.8333 \\
42.6667 \\
42.9167 \\
42.9167 \\
43.2083 \\
43.2083 \\
43.3750 \\
43.3756 \\
43.2917 \\
43.3333 \\
43.3333 \\
43.2560 \\
43.3780 \\
43.3333 \\
43.3333 \\
43.3333 \\
43.3750 \\
43.3333 \\
43.6667 \\
42.5833 \\
43.5000 \\
42.5417\end{array}$ & $\begin{array}{r}-3280 \\
4300 \\
-2350 \\
-12 C 50 \\
-6350 \\
-16225 \\
-36 C 0 \\
-6250 \\
-3400 \\
-9950 \\
-5260 \\
-2100 \\
-9450 \\
-97 C 0 \\
-27 C C \\
-2600 \\
-2080 \\
5360 \\
-9960 \\
-126.50 \\
-20400 \\
-4 c 50\end{array}$ & $\begin{array}{l}77.0427 \\
77.4167 \\
77.5030 \\
77.4583 \\
78.2500 \\
78.1250 \\
78.2667 \\
78.1250 \\
78.2667 \\
78.2083 \\
78.0417 \\
78.2917 \\
78.3750 \\
75.4167 \\
78.2917 \\
76.0833 \\
76.5833 \\
76.3333 \\
76.6833 \\
74.8333 \\
75.8333 \\
74.6750\end{array}$ & $\begin{array}{r}-4200 \\
-7100 \\
-6430 \\
-6245 \\
-2200 \\
-7375 \\
-16300 \\
-6300 \\
-2000 \\
-4300 \\
-16826 \\
-21.660 \\
-1600 \\
-9625 \\
-16700 \\
-5306 \\
-4875 \\
3806 \\
-6760 \\
-13956 \\
-11600 \\
-2250\end{array}$ & $\begin{array}{r}C \\
0 \\
0 \\
0 \\
3020 \\
0 \\
2985 \\
C \\
2528 \\
2318 \\
2366 \\
2640 \\
2655 \\
2268 \\
2165 \\
2195 \\
2501 \\
2559 \\
0 \\
0 \\
1654 \\
0\end{array}$ & $\begin{array}{r}0 \\
3671 \\
2631 \\
1921 \\
200 \\
415 \\
0 \\
0 \\
c \\
0 \\
1 \\
0 \\
0 \\
c \\
50 \\
0 \\
0 \\
0 \\
c \\
c \\
0 \\
0\end{array}$ & 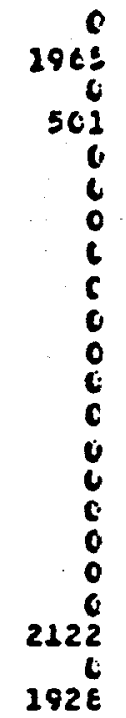 & $\begin{array}{l}858 \\
3739 \\
943 \\
914 \\
656 \\
684 \\
237 \\
247 \\
434 \\
364 \\
354 \\
561 \\
315 \\
35 \\
332 \\
305 \\
321 \\
462 \\
428 \\
1627 \\
730 \\
1262\end{array}$ \\
\hline $\begin{array}{l}4050 \\
4055 \\
4406\end{array}$ & $\begin{array}{l}240 \\
240 \\
241\end{array}$ & $\begin{array}{l}42.6667 \\
42.6667 \\
42.37 .50\end{array}$ & $\begin{array}{l}-22650 \\
-13000 \\
-2675\end{array}$ & $\begin{array}{l}75.1667 \\
74.0667 \\
76.8333\end{array}$ & $\begin{array}{l}-20000 \\
-12150 \\
-8251\end{array}$ & $\begin{array}{l}0 \\
0 \\
c\end{array}$ & c & $\begin{array}{r}2062 \\
6\end{array}$ & $\begin{array}{r}2532 \\
1899 \\
448\end{array}$ \\
\hline $\begin{array}{l}4287 \\
3946 \\
4120 \\
3899 \\
3940 \\
4682 \\
4111 \\
4203 \\
4244 \\
4606 \\
4596 \\
5095 \\
4768 \\
4428 \\
4814 \\
4378 \\
9984 \\
4524 \\
2966 \\
4224\end{array}$ & $\begin{array}{l}241 \\
242 \\
243 \\
244 \\
245 \\
245 \\
246 \\
246 \\
248 \\
249 \\
249 \\
250 \\
250 \\
257 \\
251 \\
252 \\
367 \\
253 \\
254 \\
255\end{array}$ & $\begin{array}{l}42.4167 \\
42.6667 \\
42.2917 \\
42.4167 \\
42.4583 \\
42.8750 \\
42.8750 \\
42.9167 \\
42.8333 \\
42.8750 \\
42.8750 \\
43.0427 \\
42.9167 \\
42.3333 \\
42.9167 \\
42.9583 \\
42.6667 \\
42.9583 \\
42.3333 \\
42.3333\end{array}$ & $\begin{array}{r}-24 C 0 \\
-20200 \\
-4050 \\
-5 C 0 \\
-13675 \\
-525 \\
-6300 \\
-14750 \\
-3000 \\
-50 \\
-6775 \\
-2450 \\
-7600 \\
-10075 \\
-8600 \\
-12550 \\
-13300 \\
-6600 \\
-12806 \\
-10300\end{array}$ & $\begin{array}{l}76.7683 \\
77.6 C 60 \\
77.0417 \\
76.9767 \\
76.8333 \\
76.8333 \\
76.8333 \\
76.8333 \\
76.8333 \\
76.0167 \\
76.8750 \\
76.9167 \\
76.7560 \\
77.1250 \\
76.7506 \\
76.8333 \\
77.05 C 0 \\
76.8750 \\
77.2667 \\
77.2667\end{array}$ & $\begin{array}{r}-3675 \\
-18250 \\
-5000 \\
7100 \\
-16600 \\
-1775 \\
-5800 \\
-6750 \\
-8650 \\
-1425 \\
-2050 \\
-16050 \\
-6650 \\
-6800 \\
-16000 \\
-9406 \\
-5000 \\
-3025 \\
-5300 \\
-1750\end{array}$ & $\begin{array}{l}0 \\
0 \\
0 \\
c \\
0 \\
0 \\
0 \\
c \\
0 \\
6 \\
0 \\
0 \\
0 \\
0 \\
0 \\
0 \\
c \\
0 \\
0 \\
0\end{array}$ & $\begin{array}{r}0 \\
0 \\
0 \\
0 \\
0 \\
1678 \\
1624 \\
1665 \\
1976 \\
1662 \\
2749 \\
1105 \\
1444 \\
0 \\
1556 \\
1536 \\
1427 \\
0 \\
0 \\
0\end{array}$ & $\begin{array}{r}2667 \\
0 \\
3360 \\
0 \\
1402 \\
25 \\
70 \\
75 \\
384 \\
C \\
165 \\
0 \\
6 \\
3343 \\
350 \\
46 \\
1226 \\
0 \\
2663 \\
3324\end{array}$ & $\begin{array}{l}1576 \\
1733 \\
2023 \\
470 \\
665 \\
469 \\
539 \\
534 \\
666 \\
564 \\
544 \\
400 \\
464 \\
1052 \\
478 \\
563 \\
2229 \\
403 \\
1111 \\
2327\end{array}$ \\
\hline
\end{tabular}

LAT,LONG ARE GIVEN AS DECIMAL DEGREES AND FEET.

POSITIVE VALUES INDICATE A NORTHERLY OR EASIERLY DISPLACEMENT. MEGATIVE VALUES INDICATE SCUTHERLY OR MESTERLY DISPLACEHENT. AUXILLIARY IO'S BEGINNING MITH 7 INDICATE FENNR HELLS. 
APPENDIX C (Cont)

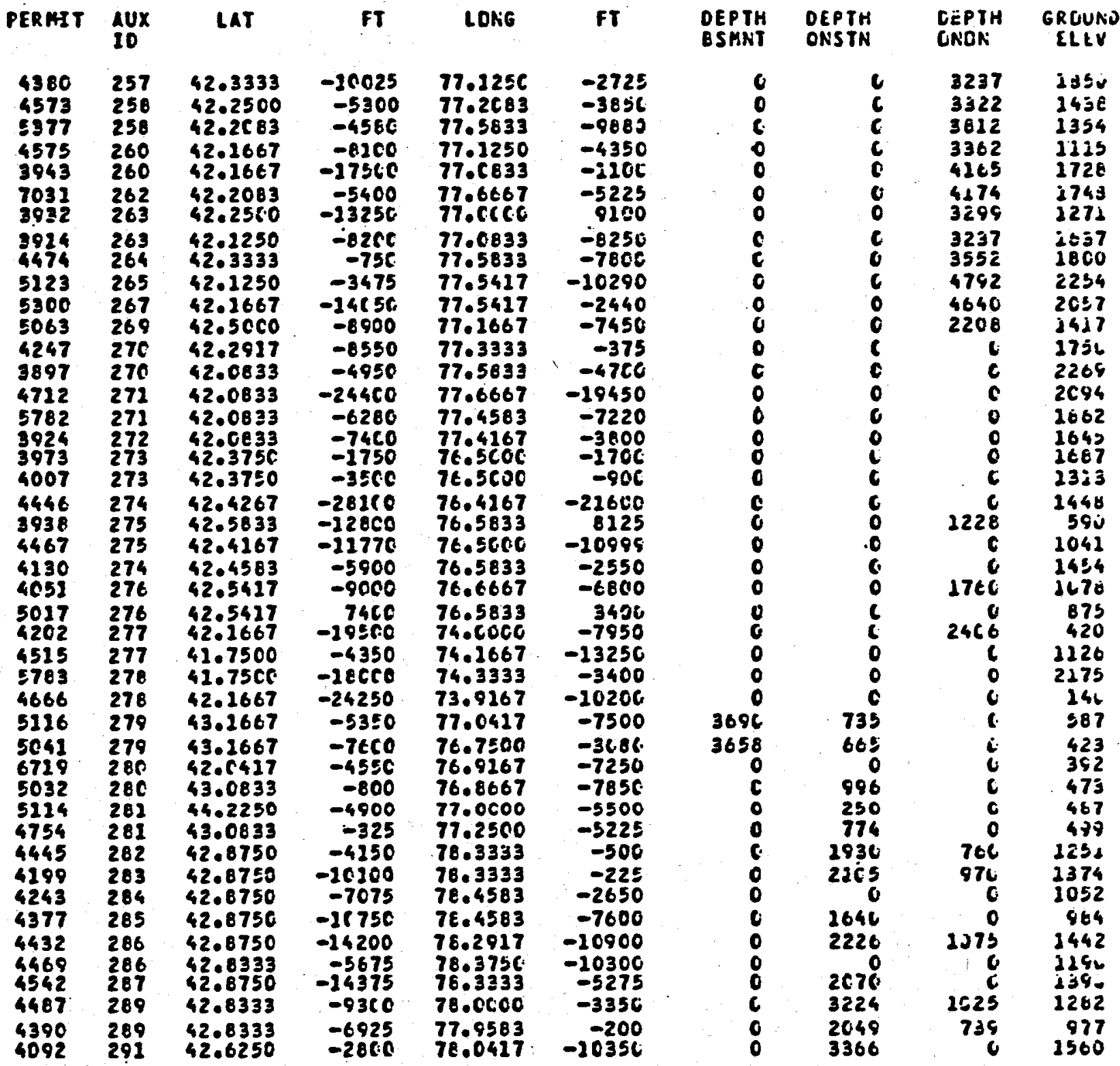

LATALONG ARE GIVEN AS DECIMAL DEgREES AND FEET. POSITIVE VALUES INDICATE A NCRTHERLY OR EASTERLY DISPLACEMENT. NEGATIVE VALUES INDICATE SCUTHERLY OR MESTERLY DISPLACEMENT. AUXILLIARY ID IS BEGINNING WITH 7 INDICATE PENAA HELLS. 
APPENDIX C (Cont)

\begin{tabular}{|c|c|c|c|c|c|c|c|c|c|}
\hline PERKIT & $\underset{I D}{A U X}$ & LAT & FT & LONG & FT & $\begin{array}{l}\text { OEPTH } \\
\text { BSMNT }\end{array}$ & $\begin{array}{l}\text { OEPTH } \\
\text { ONSTA }\end{array}$ & $\begin{array}{l}\text { DEPTH } \\
\text { GNON }\end{array}$ & GKOLPO \\
\hline $\begin{array}{l}4385 \\
4233 \\
4447 \\
4536 \\
4546 \\
4449 \\
4392 \\
4468 \\
4212 \\
6073 \\
4342\end{array}$ & $\begin{array}{l}294 \\
295 \\
296 \\
297 \\
298 \\
300 \\
301 \\
302 \\
302 \\
304 \\
304\end{array}$ & $\begin{array}{l}42.6667 \\
42.8333 \\
42.8333 \\
42.8333 \\
42.8750 \\
42.7917 \\
42.7500 \\
42.8333 \\
42.7566 \\
42.7560 \\
42.7500\end{array}$ & $\begin{array}{r}-13350 \\
-16 C 0 \\
-12275 \\
-2465 \\
-8200 \\
-1850 \\
-850 \\
-22360 \\
-1800 \\
.1910 \\
-3250\end{array}$ & $\begin{array}{l}76.0917 \\
78.0833 \\
78.1250 \\
78.1250 \\
78.1667 \\
78.1250 \\
78.1667 \\
77.9583 \\
78.6333 \\
78.1250 \\
78.3333\end{array}$ & $\begin{array}{r}-7750 \\
-9000 \\
-6725 \\
-3600 \\
-6600 \\
-2850 \\
-8350 \\
-6625 \\
-6656 \\
7340 \\
-7600\end{array}$ & $\begin{array}{l}0 \\
0 \\
0 \\
0 \\
0 \\
0 \\
0 \\
0 \\
0 \\
0 \\
0\end{array}$ & $\begin{array}{r}3368 \\
2382 \\
6 \\
2425 \\
6 \\
2592 \\
2802 \\
2319 \\
0 \\
266 C \\
2360\end{array}$ & $\begin{array}{r}0 \\
0 \\
0 \\
0 \\
0 \\
0 \\
0 \\
1630 \\
1220 \\
1380 \\
1177\end{array}$ & $\begin{array}{l}1760 \\
2510 \\
3556 \\
1501 \\
1581 \\
1580 \\
1660 \\
1224 \\
1328 \\
1561 \\
1265\end{array}$ \\
\hline $\begin{array}{l}4795 \\
4796 \\
4797 \\
4410 \\
3994 \\
3994\end{array}$ & $\begin{array}{l}305 \\
306 \\
306 \\
307 \\
307 \\
307\end{array}$ & $\begin{array}{l}42.7500 \\
42.7683 \\
42.7560 \\
42.5060 \\
42.6667 \\
42.6667\end{array}$ & $\begin{array}{r}-11650 \\
-8950 \\
-215 \\
-95 C 0 \\
-13300 \\
-133 c 0\end{array}$ & $\begin{array}{l}77.0000 \\
77.6000 \\
77.0 .100 \\
76.9267 \\
77.0000 \\
77.0000\end{array}$ & $\begin{array}{r}-5975 \\
-5975 \\
-26.36 \\
-16450 \\
-5000 \\
-5000\end{array}$ & $\begin{array}{l}0 \\
0 \\
0 \\
0 \\
0 \\
0\end{array}$ & $\begin{array}{r}2728 \\
2876 \\
2536 \\
0 \\
6 \\
0\end{array}$ & $\begin{array}{r}2032 \\
2164 \\
846 \\
0 \\
2220 \\
1226\end{array}$ & $\begin{array}{r}684 \\
960 \\
628 \\
1160 \\
990 \\
940\end{array}$ \\
\hline
\end{tabular}


APrENUIX U

SAMPLE LOCATIONS AND WATER GEOCHEMISTRY DATA

Sample Locations - Cayuga Anomaly (July, 1978)

NY001 - Baldwinville, NY depth $25 \mathrm{~m}, T=11.0^{\circ} \mathrm{C}$. Sample from doan well-tap at wellhead. Perfect collection site - well pumping. Sample about $100 \mathrm{~m}$ from river. Probably river water. Wells used because of pollution in river.

NY002 - Fulton 3. Depth $20 \mathrm{~m}, \mathrm{~T}=11.4^{\circ} \mathrm{C}$. Sample from valve at wellhead maybe $100 \mathrm{~m}$ from river alluvium.

NY003 - Fulton 6. Depth $20 \mathrm{~m}$. Sample from valve at wellhead - maybe $100 \mathrm{~m}$ from river. Just about 1 mile from NY002 the bear wells are $37 \mathrm{~m}$ but cannot be sampled.

NY004 - Bear well - still at Fulton - their deepest well may be $200 \mathrm{~m}$ from river. Over $22 \mathrm{~m}$ deep, 120 sample baled from casing - small lead.

NY005 - Near Fleming. Dug well about $9 \mathrm{~m}$ deep, sample taken from tap at farm house. $T=16.2^{\circ} \mathrm{C}$. Temp is not valid due to sampling conditions. About $1.6 \mathrm{~km}$ west of Fleming on $34 \mathrm{~B}$.

NY006 - $3.2 \mathrm{~km}$ out of Fleming on 34B. T-14.6 $6^{\circ} \mathrm{C}$. May be bad water from $62 \mathrm{~m}$ well to pressure tank - sample from tap at house - Guliver Ranch.

Nr007 - Scipioville - well about $62 \mathrm{~m}$ deep. Sample from house tap - well to tank to tap. $T=17.0^{\circ} \mathrm{C}$ but probably bad temp. About $1.6 \mathrm{~km}$ out of Scipioville - shale.

NY008 - About $4.8 \mathrm{~km}$ south of Genoa at Baun Egg Farm. Well $85 \mathrm{~m}$ deep. Sample from valve in bldg. $\mathrm{T}=12.8^{\circ} \mathrm{C}$ suspension - well goes to pipe - sample from pipe.

NY009 - City of Homer - Newton pump station 3. Well $25 \mathrm{~m}$ deep behind city water works. Well pumping and sample from tap - good temp a $8.8^{\circ} \mathrm{C}$.

NY010 - Sempronius - Well $39 \mathrm{~m}$ deep at pump house. Sample from tap at wellhead. Hopkins farm.

NY011 - Spring at Sempronius - dug well $2 \mathrm{~m}$ deep. Then siphoned down to lake by rubber tube. $T=13.0^{\circ} \mathrm{C}$. Spring \& tube flow continuously. Hopkins lumber

NY012 - On hill about $3.2 \mathrm{~km}$ east of rd jct to Owasco on $38 \mathrm{~A}$ above the lake $17.0^{\circ} \mathrm{C}$. Questionable. Well $49 \mathrm{~m}$ deep to pressure pump to lawn tap for samples. E. Niles.

NY013 - $1.6 \mathrm{~km}$ east on U.S. 20 from junction with 174 to Navarino - on top of hill - depth of well not known - iron \& hard water rumored. T $12.0^{\circ} \mathrm{C}$. Water from tap, but run long enough to drain pipe. Marcellus. 
NY014 - At intersection of county line with Hwy 79 at Mecklenburg. Well $23 \mathrm{~m}$ deep. Pressure pump \& tap in barn. $18.4^{\circ} \mathrm{C}$.

Nro15 - $3.2 \mathrm{~km}$ west of Interlaken on 96A. Dug well to $6 \mathrm{~m}$. Standing water in well pumped to tank to garden hose for sample. $T=22.8^{\circ} \mathrm{C}$. High even though hot water from hose was drained.

NY016 - Just north of Romulus at dairy farm. Dug well $8 \mathrm{~m}$ deep. Water table at $4 \mathrm{~m}$ depth. $T=12.8^{\circ} \mathrm{C}$. Standing water in well - pumped from depth - hose drained.

NY017 - $3.2 \mathrm{~km}$ north of Waterloo on 96 . Well $32 \mathrm{~m}$ deep and reported to be very hard water. $T=14^{\circ} \mathrm{C}$. Water pumped to tank to garden hose for our sample.

NY018 $-3.2 \mathrm{~km}$ west of Flint on U.S. 20. Pumped well depth unknown. Sample at tap at side of house. $T=17.8^{\circ}$.

NY019 - $1.6 \mathrm{~km}$ south of Townsend on 329. Well shallow-hand dug maybe $10 \mathrm{~m}$. Well pumped to lawn hose for sample $14^{\circ} \mathrm{C}$. In mountains shaleoutcrops. Precisely $5.6 \mathrm{~km}$ from Townsend.

NY021 - Just east of Prattsburg on unnamed highway; Well $37 \mathrm{~m}$ deep - sample from stock tank by lifting float valve \& getting water. 


\section{APPENDIX D (Cont)}

EAST AURORA ANOMALY: COMPARISON OF LABORATORY ANALYSES

Three samples were analyzed by the State University of New York at Buffalo Geology Lab and New Mexico State University Lab for comparison of results as shown below:

\begin{tabular}{|c|c|c|c|c|c|c|}
\hline & $\begin{array}{l}\text { New Mexico } \\
\text { Sample } 781\end{array}$ & $\begin{array}{l}\text { SUNY } \\
\text { NYOOI }\end{array}$ & $\begin{array}{l}\text { New Mexico } \\
\text { Sample } 782\end{array}$ & $\begin{array}{l}\text { SUNYY } \\
\text { NYO05 }\end{array}$ & $\begin{array}{l}\text { New Mexico } \\
\text { Sample } 783\end{array}$ & $\begin{array}{l}\text { SUNY } \\
\text { NYOIO }\end{array}$ \\
\hline ph & 8.09 & 7.6 & 8.16 & 7.5 & 7.92 & 7.7 \\
\hline Ca & 59.3 & 57.0 & 85.0 & 87.2 & 42.3 & 41.7 \\
\hline $\mathrm{Mg}$ & 25.3 & 26.4 & 23.2 & 24.4 & 8.1 & 8.0 \\
\hline $\mathrm{Na}$ & 5.3 & 5.0 & 44.1 & 40.7 & 3.7 & 3.6 \\
\hline$K$ & 0.8 & 0.7 & 0.8 & 0.7 & 0.4 & 0.4 \\
\hline Cl & 15.2 & 11 & 77.3 & 78 & 4.2 & 2.7 \\
\hline $\mathrm{CO}_{3}$ & 0 & 0 & 0 & 0 & 0 & 0 \\
\hline $\mathrm{HCO}_{3}$ & 242.8 & 252 & 322.2 & 322 & 150.1 & 149 \\
\hline $\mathrm{SO}_{4}$ & 31.2 & 25 & 37.0 & 35 & 20.2 & ig \\
\hline $\mathbf{F}$ & $<.20$ & .09 & $<.20$ & .08 & $<.20$ & .07 \\
\hline $\mathrm{Fe}$ & $<.10$ & .08 & $<.10$ & .04 & $<.10$ & .04 \\
\hline $\mathrm{SiO}_{2}$ & 9.75 & 9.25 & 6.5 & 5.95 & 7.0 & 6.85 \\
\hline $\mathrm{NO}_{3}$ & 16.5 & 14 & 17.5 & 16 & 11.0 & 3.5 \\
\hline
\end{tabular}


APPENDIX D (Cont)

RESULTS OF CHEMICAL ANALYSIS

\begin{tabular}{|c|c|c|c|c|c|c|c|c|c|}
\hline & mo01 & $\mathbf{x + 0 0 2}$ & mroo3 & arood & moos & $17 \times 006$ & Eroo7 & rroos & iroog \\
\hline $\mathrm{suO}_{2}$ & $\begin{array}{l}0.342 \\
0.2 \times 2\end{array}$ & $\begin{array}{l}10.012 \\
20.3 \times 2\end{array}$ & $\begin{array}{l}10.5 \mathrm{n} 2 \\
20.2 \mathrm{n} 2\end{array}$ & $\begin{array}{l}0.022 \\
0.112\end{array}$ & $\begin{array}{l}6 . \cos 2 \\
5.2012\end{array}$ & $\begin{array}{l}8.022 \\
7.0002\end{array}$ & $\begin{array}{l}16.6 \mathrm{ng} \\
16.1112\end{array}$ & $\begin{array}{l}9.6 \times 2 \\
7.7 \mathrm{w2}\end{array}$ & $\begin{array}{l}3.1122 \\
5.1 \mathrm{2} 2\end{array}$ \\
\hline $\begin{array}{l}\text { Soen } 1500 \\
\left(\sin 1 \cos ^{\prime}\right.\end{array}$ & 90 & 10 & 60 & 10 & 10 & 60 & 600 & 90 & 30 \\
\hline $\begin{array}{r}100 \\
102 \\
\end{array}$ & $\begin{array}{l}43.9 \\
43.8\end{array}$ & $\begin{array}{l}31.7 \\
31.5\end{array}$ & $\begin{array}{l}26.1 \\
25.9\end{array}$ & $\begin{array}{l}33.2 \\
32.8\end{array}$ & $\begin{array}{l}40.5 \\
40.6\end{array}$ & $\begin{array}{l}3.0 \\
2.5\end{array}$ & $\begin{array}{l}36.3 \\
55.8\end{array}$ & $\begin{array}{l}7.9 \\
7.0\end{array}$ & $\begin{array}{l}17.3 \\
17.3\end{array}$ \\
\hline $\begin{array}{r}210 \quad 12 \\
12\end{array}$ & $\begin{array}{l}79.5 \\
10.0\end{array}$ & $\begin{array}{l}232 . \\
133 .\end{array}$ & $\begin{array}{l}79.7 \\
78.2\end{array}$ & $\begin{array}{l}53.0 \\
53.9\end{array}$ & $\begin{array}{l}122 . \\
222 .\end{array}$ & $\begin{array}{l}6.9 \\
6.6\end{array}$ & $\begin{array}{l}73.6 \\
74.1\end{array}$ & $\begin{array}{l}22.7 \\
20.9\end{array}$ & $\begin{array}{l}76.1 \\
25.2\end{array}$ \\
\hline$\frac{10}{120}$ & $\begin{array}{l}6.6 \\
6.0\end{array}$ & $\begin{array}{l}87.5 \\
87.4\end{array}$ & $\begin{array}{l}26.5 \\
25.0\end{array}$ & 6.4 & $\begin{array}{l}54.9 \\
55.0\end{array}$ & $\begin{array}{l}155 . \\
155 .\end{array}$ & $\begin{array}{l}27.0 \\
26.8\end{array}$ & $\begin{array}{l}229 . \\
233\end{array}$ & $\begin{array}{l}9.7 \\
9.6\end{array}$ \\
\hline $\begin{array}{r}Z_{2}^{0} \\
\therefore\end{array}$ & $\begin{array}{l}0.8 \\
2.2\end{array}$ & $\begin{array}{l}4.4 \\
4.7\end{array}$ & $\begin{array}{l}2.0 \\
2.1\end{array}$ & $\begin{array}{l}1.6 \\
1.5\end{array}$ & $\begin{array}{l}0.9 \\
1.0\end{array}$ & $\begin{array}{l}1.7 \\
1.6\end{array}$ & $\begin{array}{l}1.6 \\
1.6\end{array}$ & $\begin{array}{l}2.0 \\
2.0\end{array}$ & $\begin{array}{l}0.8 \\
0.8\end{array}$ \\
\hline $\begin{array}{c}\text { Carboenta } \\
\text { un }\end{array}$ & 0 & 0 & 0 & 0 & - & 0.7 & 0 & 0 & e \\
\hline $\begin{array}{c}\text { Irearbonate } \\
\text { mI }\end{array}$ & 252 & 253 & 219 & 260 & 322 & 210 & 331 & 312 & 192 \\
\hline $\begin{array}{r}\text { Flooride } \\
\mathrm{mr}_{2}\end{array}$ & 0.09 & 0.11 & 0.08 & 0.07 & 0.08 & 0.27 & 0.82 & 0.64 & 0.05 \\
\hline $\begin{array}{r}\text { Drortida } \\
12\end{array}$ & 11. & 193 & 41. & 4. & 78. & 3. & 1.4 & 220. & 6.1 \\
\hline Exphate & $\begin{array}{ll}26 \times 2 & 2412 \\
37 m 2 & 25 \times 2\end{array}$ & $\begin{array}{l}34 \mathrm{N2} \\
33992\end{array}$ & $\begin{array}{l}19252 \\
1792\end{array}$ & $\begin{array}{c}372 \\
37 \times 23922\end{array}$ & $\begin{array}{l}35: 22 \\
39: 2\end{array}$ & 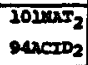 & $\begin{array}{l}46 \times 2 \\
55 \times 2\end{array}$ & $12 x=2$ & 1ha 2 \\
\hline itrate & 14 & 4.0 & 0.2 & 2.4 & 16 & 1.9 & 2.3 & 2.4 & 14 \\
\hline $\begin{array}{c}\text { Initinil } \\
\text { sottie ph } \\
\text { nn }\end{array}$ & 7.6 & 7.4 & 7.5 & 7.5 & 7.5 & 2.6 & 7.4 & 7.6 & 7.6 \\
\hline
\end{tabular}

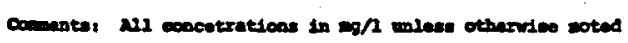

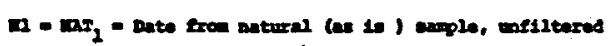

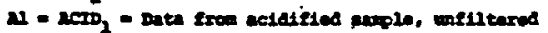

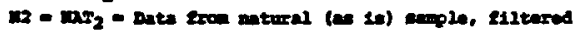

\begin{tabular}{|c|c|c|c|c|c|c|c|c|c|}
\hline & rropo & mo11 & rro12 & mros3 & mo14 & rro13 & aro216 & irro17 & tyo18 \\
\hline$=10_{2}$ & $\begin{array}{l}6.5 \times 2 \\
7.2 \times 2\end{array}$ & $\begin{array}{l}7.012 \\
7.712\end{array}$ & $\begin{array}{l}22.2 \times 2 \\
22.3 \times 2\end{array}$ & $\begin{array}{l}12.122 \\
11.622\end{array}$ & 20.212 & $\begin{array}{l}0.212 \\
0.0022\end{array}$ & $\begin{array}{l}12.2122 \\
12.3192\end{array}$ & $\begin{array}{l}10.7 \times 2 \\
10.5 \mathrm{n} 2\end{array}$ & $\begin{array}{l}9.1 \times 2 \\
9.3 \times 2\end{array}$ \\
\hline $\begin{array}{l}\text { rotan } \operatorname{lron} \\
(\mathrm{g} / \mathrm{ma}) \max \end{array}$ & 40 & 100 & 70 & 400 & 70 & 10 & 70 & 210 & 220 \\
\hline $\begin{array}{rl}20 & 22 \\
n\end{array}$ & $\begin{array}{l}13.3 \\
13.2\end{array}$ & $\begin{array}{l}14.2 \\
14.0\end{array}$ & $\begin{array}{l}33.9 \\
33.7\end{array}$ & $\begin{array}{l}55.855 .0 \\
55.554 .3\end{array}$ & $\begin{array}{l}.2 \\
e .2\end{array}$ & $\begin{array}{l}29.6 \\
29.1\end{array}$ & $\begin{array}{l}56.955 .9 \\
58.457 .6\end{array}$ & $\begin{array}{l}159 \\
160\end{array}$ & $\begin{array}{l}44.6 \\
45.5\end{array}$ \\
\hline $\begin{array}{l}2 \\
m 2\end{array}$ & $\begin{array}{l}38.3 \\
38.3\end{array}$ & $\begin{array}{l}74.4 \\
74.6\end{array}$ & $\begin{array}{l}67.1 \\
66.7\end{array}$ & $\begin{array}{ll}123 & 122 \\
200 & 102\end{array}$ & 30.5 & $\begin{array}{l}214 \\
211\end{array}$ & $\begin{array}{ll}156 & 152 \\
163 & 149\end{array}$ & 719 & $\begin{array}{l}117 \\
117\end{array}$ \\
\hline \begin{tabular}{|l|l|}
$m_{2}{ }^{\circ}$ & $m$ \\
$m$
\end{tabular} & $\begin{array}{l}4.0 \\
4.8\end{array}$ & $\begin{array}{l}2.3 \\
2.3\end{array}$ & $\begin{array}{l}38.7 \\
30.0\end{array}$ & $\begin{array}{l}6.05 .9 \\
5.55 .4\end{array}$ & $\begin{array}{l}131 \\
131\end{array}$ & $\begin{array}{l}68.4 \\
66.4\end{array}$ & 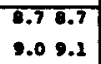 & $\begin{array}{l}133 \\
134\end{array}$ & $\begin{array}{l}15.3 \\
13.9\end{array}$ \\
\hline 12 & $\begin{array}{l}0.5 \\
0.6\end{array}$ & $\begin{array}{l}0.5 \\
0.6\end{array}$ & $\begin{array}{l}1.6 \\
1.8\end{array}$ & $\begin{array}{ll}1.0 & 1.0 \\
1.2 & 1.2\end{array}$ & $\begin{array}{l}3.3 \\
2.3\end{array}$ & $\begin{array}{l}3.4 \\
3.6\end{array}$ & $\begin{array}{lll}1.9 & 1.9 \\
2.2 & 2.2\end{array}$ & $\begin{array}{l}5.7 \\
5.9\end{array}$ & $\begin{array}{l}9.6 \\
10.5\end{array}$ \\
\hline $\begin{array}{c}\text { Carbonate } \\
\text { in }\end{array}$ & 0 & 0 & 0 & 0 & 0 & 0 & 0 & 0 & 0 \\
\hline $\begin{array}{c}\text { Bearbonite } \\
\text { w1 }\end{array}$ & 149 & 248 & 305 & 349 & 305 & 462 & 248 & 99 & 309 \\
\hline Fooride & 0.07 & 0.05 & 0.20 & 0.19 & 0.27 & 0.21 & 0.16 & 0.83 & 0.12 \\
\hline Croride & 2.7 & 20.8 & 2.1 & 3. & 23. & 21. & 43. & 60. & 14. \\
\hline Eolphete & 19:2 & 23102 & $\begin{array}{l}21102 \\
22 \times 2\end{array}$ & 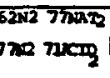 & 20 & $24 \times 2$ & $97 \mathrm{ACDO}_{2}$ & $\begin{array}{l}1790 \times \mathrm{CD}_{2} \\
1720 \mathrm{CD} \mathrm{D}_{2}\end{array}$ & $\begin{array}{l}\operatorname{sen} 2 \\
\operatorname{sen} 2\end{array}$ \\
\hline Antrate & 3.5 & 25 & 0.2 & 0.2 & 0.2 & 0.0 & 0.0 & 0.2 & क. \\
\hline $\begin{array}{l}\text { Initial } \\
\text { bottle po }\end{array}$ & 7.7 & 7.7 & 7.6 & 7.5 & 7.7 & 8.3 & 7.5 & 7.3 & 7.6 \\
\hline
\end{tabular}


APPENDIX D (Cont)

RESULTS OF CHEMICAL ANALYSIS

\begin{tabular}{|c|c|c|c|}
\hline & Exo29 & mro20 & rro21 \\
\hline $\mathrm{sio}_{2}$ & $\begin{array}{l}20.6 \mathrm{a2} \\
11.3 \times 2\end{array}$ & $\begin{array}{l}9.4 .22 \\
9.2112\end{array}$ & $\begin{array}{l}27.022 \\
26.582\end{array}$ \\
\hline $\begin{array}{l}\text { Total iron } \\
\text { ( } \mu \mathrm{g} / \mathrm{ml}) \text { ) }\end{array}$ & 200 & $<200$ & 60 \\
\hline$x_{200}$ & $\begin{array}{l}26.617 .4 \\
20.621 .5\end{array}$ & $\begin{array}{l}12.4 \\
12.4\end{array}$ & $\begin{array}{l}41.181 .2 \\
39.0 \quad 38.6\end{array}$ \\
\hline $\begin{array}{r}\mathbf{M O} \\
\vdots \quad \mathbf{m}\end{array}$ & $\begin{array}{l}54: 353.7 \\
67.257 .8\end{array}$ & $\begin{array}{l}39.3 \\
39.1\end{array}$ & $\begin{array}{l}99.999 .5 \\
72.462 .8\end{array}$ \\
\hline $\begin{array}{rr}\operatorname{Ra}_{2} \mathrm{O} & \mathbf{1 2} \\
\ddots & 12\end{array}$ & $\begin{array}{c}67103 \\
61.461 .4\end{array}$ & $\begin{array}{l}28.4 \\
28.2\end{array}$ & $\begin{array}{l}31.331 .6 \\
30.030 .1\end{array}$ \\
\hline $\begin{array}{ll}\boldsymbol{Z}^{0} & \mathbf{M 2} \\
\mathbf{m 2}\end{array}$ & $\begin{array}{l}2.91 .8 \\
1.91 .9\end{array}$ & $\begin{array}{l}0.8 \\
1.2\end{array}$ & $\begin{array}{l}2.52 .5 \\
2.62 .7\end{array}$ \\
\hline $\begin{array}{r}\text { Carbonate } \\
\mathrm{n2}\end{array}$ & 0 & 0 & 0 \\
\hline $\begin{array}{c}\text { Mcarbonate } \\
\text { m2 }\end{array}$ & $=304$ & 170 & 355 \\
\hline $\begin{array}{l}\text { Inoxide } \\
\text { N2 }\end{array}$ & 0.19 & 0.23 & 0.15 \\
\hline $\begin{array}{r}\text { chloride } \\
\text { H2 }\end{array}$ & 14. & 3. & 2.3 \\
\hline Sulphate & $\cos 2$ & $22 \times 2$ & $\begin{array}{l}16 \sqrt{2} \\
\cos 2\end{array}$ \\
\hline iifrate & 0.2 & 0.5 & 2.3 \\
\hline $\begin{array}{l}\text { initial } \\
\text { bottle pr }\end{array}$ & 7.4 & 7.6 & 7.6 \\
\hline
\end{tabular}

CHEMISTRY OF THE WATER SAMPLES -FOR EAST AURORA ANOMOLY

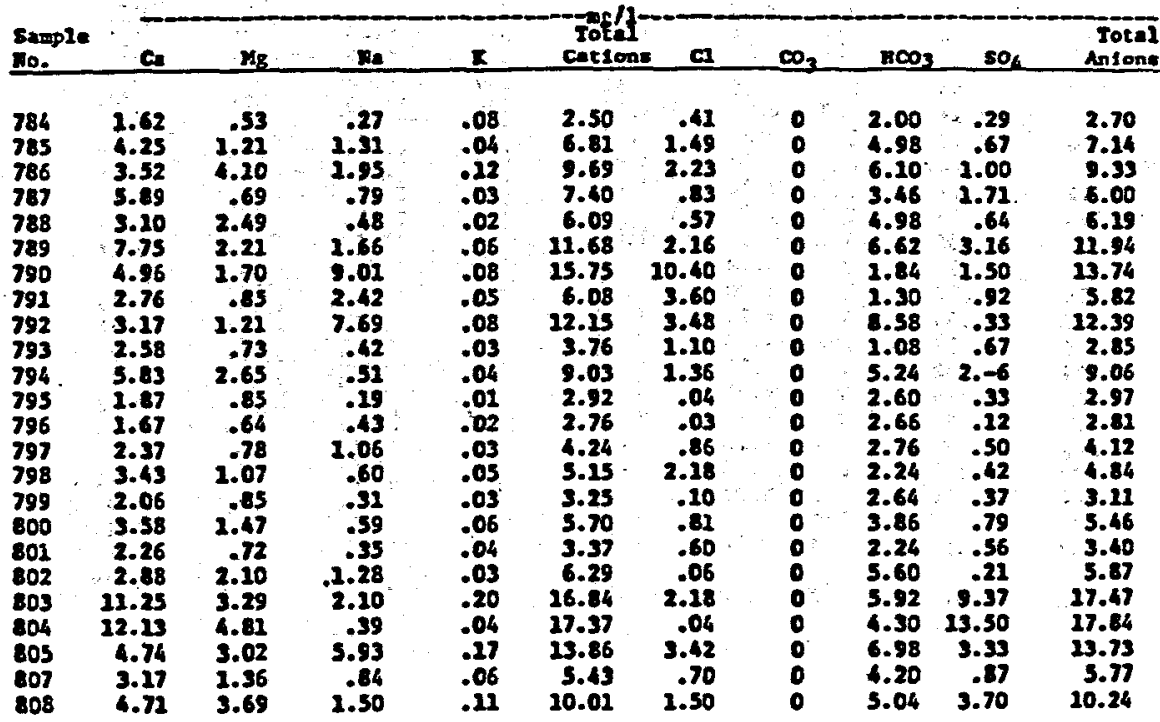


APPENDIX D (Cont)

CHEMISTRY OF THE WATER SAMPLES FOR EAST AURORA ANOMOLY

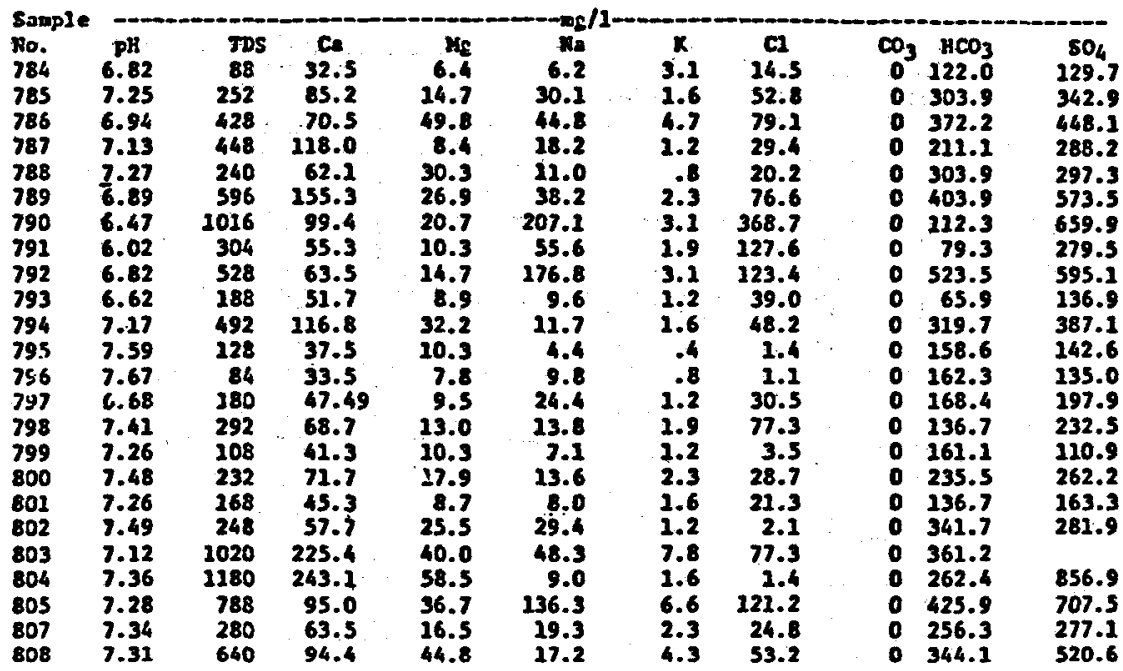

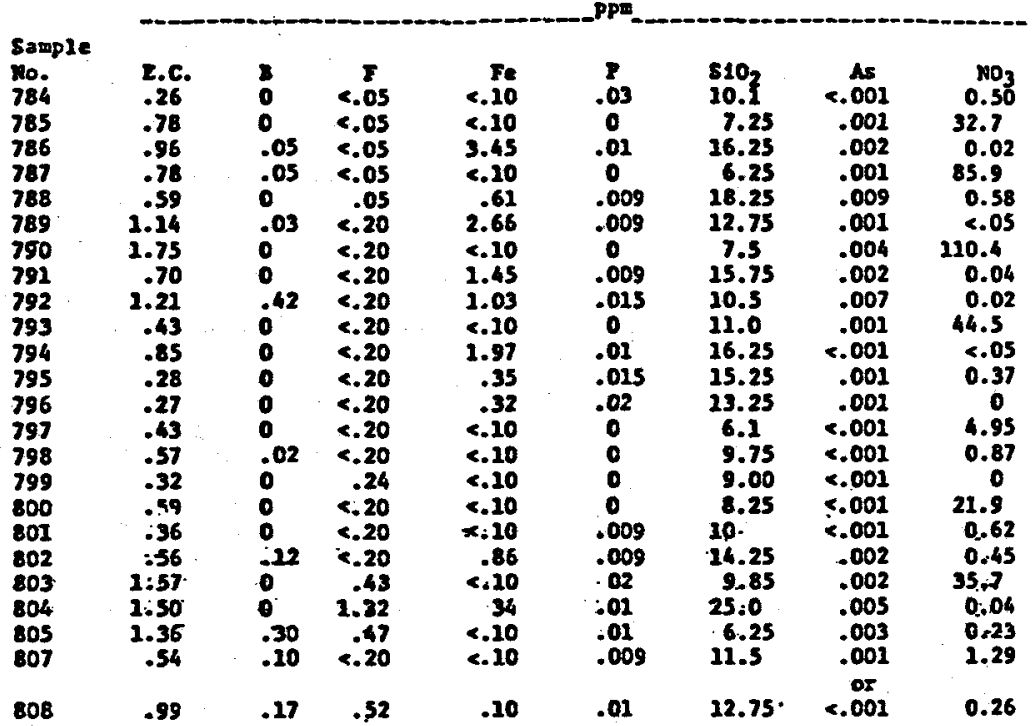


APPENDIX D (Cont)

RESULTS OF CHEMICAL ANALYSIS

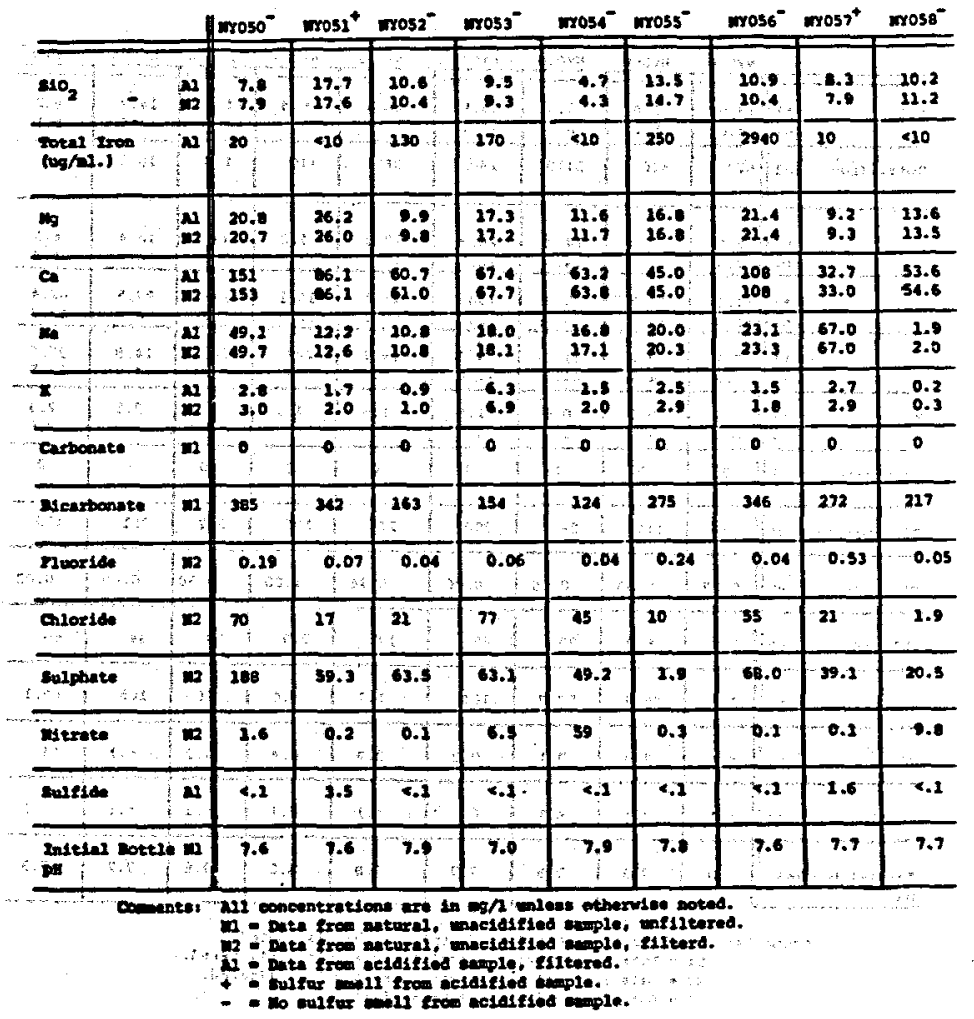

\begin{tabular}{|c|c|c|c|c|c|c|c|c|c|}
\hline & reose- & $20060^{-}$ & noos & $0 \times 062^{-}$ & $\mathrm{Hros}^{-}$ & moos & $\operatorname{mos}^{\circ}$ & $\operatorname{mros}^{+}$ & sros \\
\hline $\mathrm{so}_{2}$ & 7.2 & $\begin{array}{l}7.5 \\
7.0\end{array}$ & $\begin{array}{l}20.6 \\
20.1\end{array}$ & $\begin{array}{l}16.0^{\circ} \\
15.0^{\circ}\end{array}$ & $\begin{array}{l}13.2 \\
12.7\end{array}$ & $\begin{array}{l}12: 4 \\
11 ; 9\end{array}$ & $\begin{array}{l}0.6 \\
7.6 \\
\end{array}$ & $\begin{array}{l}12.9 \\
12.5\end{array}$ & $\begin{array}{r}9.9 \\
9.7 \\
\end{array}$ \\
\hline 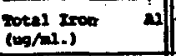 & $k 10$ & 200 & 100 & 490 & 260 & 2870 & 2730 & Aso 6 & 30 \\
\hline a. & $\begin{array}{l}18.7 \\
28.6 \\
\end{array}$ & $\begin{array}{l}27.6 \\
22.8\end{array}$ & $\begin{array}{l}14.7 \\
13.4\end{array}$ & $\begin{array}{r}29.3 \\
29.2 \\
\end{array}$ & $\begin{array}{l}2.2 \\
22.0\end{array}$ & $\begin{array}{r}23.1 \\
13.4 \\
\end{array}$ & $\begin{array}{l}22.3 \\
22.6\end{array}$ & $\begin{array}{l}21.7 \\
28.6 \\
\end{array}$ & $\begin{array}{l}14.5 \\
14.5 \\
\end{array}$ \\
\hline a1 & $\begin{array}{l}92.5 \\
92.0 \\
\end{array}$ & $\begin{array}{l}102 \\
102\end{array}$ & $\begin{array}{r}4.4 . \\
42.1 \\
\end{array}$ & $\begin{array}{l}9.1 \\
89.0\end{array}$ & $\begin{array}{l}131 \\
132 \\
\end{array}$ & $\begin{array}{r}43.8 \\
45.2 \\
\end{array}$ & 12.3 & 34.7 & 63.5 \\
\hline as & $\begin{array}{l}0.3 \\
0.4- \\
\end{array}$ & $\begin{array}{l}12.7 \\
13.0 \\
\end{array}$ & $\begin{array}{c}212 \\
2146\end{array}$ & $\begin{array}{ll}23.2 \\
23.0\end{array}$ & $\begin{array}{l}25.7 \\
25.6\end{array}$ & $\begin{array}{l}30.5 \\
32.5 \\
\end{array}$ & $\begin{array}{l}5.2 \\
5.4\end{array}$ & $\begin{array}{r}31.0 \\
31.6 \\
\end{array}$ & 43.8 \\
\hline 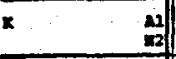 & $\begin{array}{l}1.4 \\
1.6 \\
\end{array}$ & $\begin{array}{l}2.1 \\
2.6 \\
\end{array}$ & $\begin{array}{r}3.0 \\
3.4 \\
\end{array}$ & 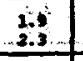 & $\begin{array}{l}2.1 \\
2.2\end{array}$ & 2.2 & $\begin{array}{l}0.8 \\
5.5 \\
\end{array}$ & $\begin{array}{l}3.2 \\
3.6 \\
\end{array}$ & $\begin{array}{r}2.0 \\
2.1 \\
\end{array}$ \\
\hline Carbonet: & 0 & $\circ$ & 0 & 0 & 0 & i. & $\sigma$ & 6 & $=0$ \\
\hline Mearbonate $m$ & 269 & 324 & 468 & 268 & 332 & 191 & 154 & 301 & 278 \\
\hline Fuorien & 0.05 & 0.00 & 0.31 & 6.09 & 0.08 & Q.j7 & 0.07 & 0.15 & 0.27 \\
\hline chloride & 31 & 44 & 241 & $33^{5}: 5$ & 46 & 43 & 6.2 & 64 & 1 \\
\hline Swlphate & 29.7 & $\bullet$ & 0.0 & 26 & 2187 & $3 \hat{3}, e^{2}$ & 10.0 & 18.9 & 11.7 \\
\hline nitrete & 56 & 0.6 & 0.4 & 6.2 & 0.5 .5 & 2.3 & 0.2 & 0.2 & 0.5 \\
\hline contsde & $<.2$ & 4.2 & -41 & 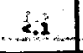 & $\therefore 1^{-}=$ & 8.1 & 5 & $+5^{3}$ & $\therefore 2$ \\
\hline $\begin{array}{l}\text { Inithes cottle wh } \\
\text { pla }\end{array}$ & 7.4 & 7.6 & $\bullet$ & 3.6 & 9.6 & & 7.0 & $7.4 \div 4$ & 7.8 \\
\hline
\end{tabular}

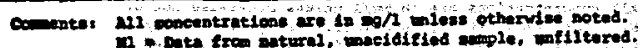

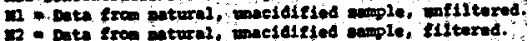

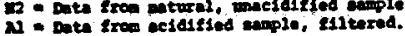




\section{APPENDIX D (Cont)}

RESULTS OF CHEMICAL ANALYSIS

\begin{tabular}{|c|c|c|c|c|c|c|c|c|c|}
\hline & mose- & mros: & mroro & $\mathrm{mron}^{*}$ & $2 \times 072^{-}$ & $\mathrm{wr}^{2073^{+}}$ & $5 \times 074^{-}$ & mro7s" & m2076 \\
\hline $\begin{array}{r}n \\
-\quad 1\end{array}$ & $\begin{array}{l}14.9 \\
14.0\end{array}$ & s.: & $\begin{array}{l}7.3 \\
6.7\end{array}$ & $\begin{array}{l}24.9 \\
14.4\end{array}$ & $\begin{array}{l}13.5 \\
22.9\end{array}$ & $\begin{array}{l}6.3 \\
6.1\end{array}$ & $\begin{array}{l}22.6 \\
12.6\end{array}$ & $\begin{array}{l}24.7 \\
24.4\end{array}$ & $\begin{array}{l}0.2 \\
.1 .2\end{array}$ \\
\hline $\begin{array}{ll}\text { 2otal 2roin } \\
(09 / 21 .)\end{array}$ & 1620 & 40 & 5110 & 200 & 300 & $<20$ & 270 & 1010 & 410 \\
\hline 魚) & $\begin{array}{l}22.8 \\
12.7\end{array}$ & $\begin{array}{l}7.3 \\
7.3 \\
\end{array}$ & $\begin{array}{r}24.3 \\
24.5 \\
\end{array}$ & $\begin{array}{l}28.3 \\
28.3 \\
\end{array}$ & $\begin{array}{l}22.0 \\
21.0 \\
\end{array}$ & $\begin{array}{l}5.0 \\
4.9 \\
\end{array}$ & $\begin{array}{l}33.7 \\
32.7 \\
\end{array}$ & $\begin{array}{l}16.4 \\
26.3 \\
\end{array}$ & $\begin{array}{l}6.5 \\
6.4 \\
\end{array}$ \\
\hline $\begin{array}{l}\mathbf{2} \\
\mathbf{m} \\
\end{array}$ & $\begin{array}{l}4.2 \\
42.6\end{array}$ & $\begin{array}{l}32.2 \\
33.2 \\
\end{array}$ & $\begin{array}{l}7.2 \\
7.2 \\
\end{array}$ & $\begin{array}{l}234 \\
234 \\
\end{array}$ & $\begin{array}{r}46.2 \\
40.2 \\
\end{array}$ & $\begin{array}{l}26.1 \\
26.3 \\
\end{array}$ & $\begin{array}{l}179 \\
176 \\
\end{array}$ & $\begin{array}{r}97.5 \\
0.9 \\
\end{array}$ & $\begin{array}{l}62.4 \\
68.7 \\
\end{array}$ \\
\hline and & $\begin{array}{l}7.1 \\
\mathbf{7 . 3} \\
\end{array}$ & 3.1 & 21.0 & 20.9 & $\begin{array}{l}19.2 \\
29.4 \\
\end{array}$ & 290 & $\begin{array}{l}26.3 \\
26.1 \\
\end{array}$ & $\begin{array}{l}24.8 \\
14.6\end{array}$ & $\begin{array}{l}27.8 \\
27.5 \\
\end{array}$ \\
\hline $\begin{array}{l}\mathbf{M} \\
\mathbf{n}\end{array}$ & $\begin{array}{l}0.7 \\
0.9\end{array}$ & $\begin{array}{l}0.7 \\
1.0\end{array}$ & $\begin{array}{l}0.6 \\
1.0 \\
\end{array}$ & $\begin{array}{l}2.0 \\
2.2 \\
\end{array}$ & 2.6 & $\begin{array}{l}4.3 \\
4.3 \\
\end{array}$ & 3.7 & $\begin{array}{l}2.5 \\
2.7 \\
\end{array}$ & $\begin{array}{l}2.3 \\
2.6 \\
\end{array}$ \\
\hline carbonate & - & 0 & - & 0 & 0 & - & 0 & 0 & 0 \\
\hline Mcurbenate ma & 209 & 211 & 242 & 398 & 245 & 595 & 362 & 251 & 210 \\
\hline Flvoride & 0.07 & 0.03 & 0.05 & 0.06 & 0.34 & 2.00 & 0.38 & 0.09 & 0.02 \\
\hline Crloride & 6.9 & 6.9 & 29 & 49 & 17 & 249 & 69 & 34 & 66 \\
\hline auphete & 1.6 & 26.2 & se.7 & 204 & 1.7 & 4.6 & 266 & 205 & 65.1 \\
\hline milrate. & $<.1$ & 2.0 & 0.1 & 0.2 & 0.2 & 0.4 & 0.2 & $<.1$ & 24 \\
\hline sulfsele & $<.1$ & $<.1$ & $<.1$ & 0.8 & $<.1$ & 0.1 & $<.1$ & $<.1$ & $<.1$ \\
\hline instes motenon & e.0 & 7.9 & 7.5 & 7.7 & 7.0 & 8.0 & 7.6 & 7.2 & 7.3 \\
\hline
\end{tabular}

coments: concentrations are in ma/ anlost otherwise noted.

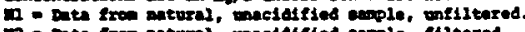

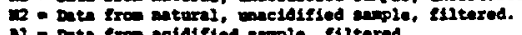

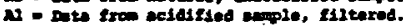

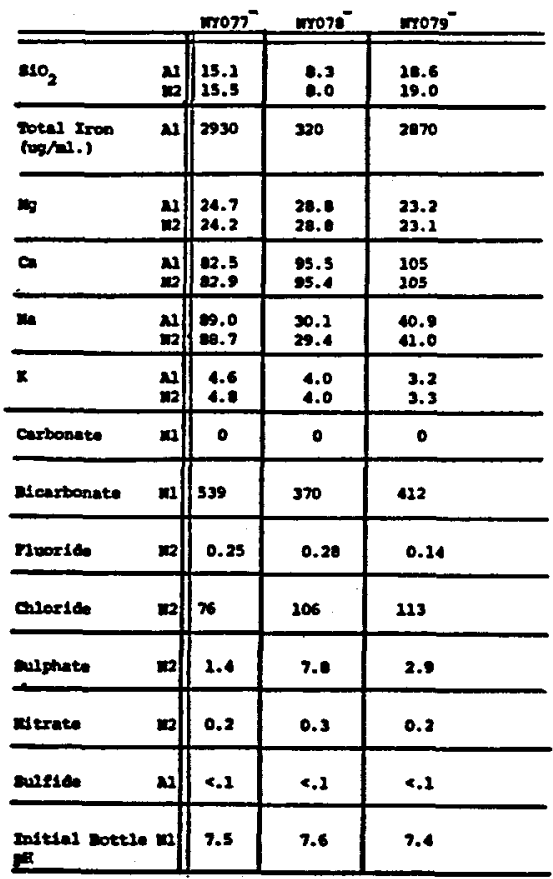

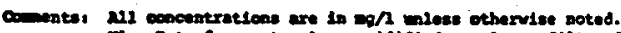

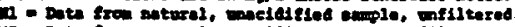

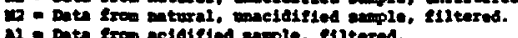

